\title{
MDI: Mathematica Database Interface for the MFEDB
}

\author{
J. C. Wiley, W. H. Miner, Jr. and D. W. Ross \\ Fusion Research Center \\ DOE/ER/53266--39 \\ DE92 014337 \\ The University of Texas at Austin \\ Austin, Texas 78712
}

April 1992

\begin{abstract}
We describe a new interface for the Magnetic Fusion Energy Database, MFEDB, which uses Mathematica ${ }^{\circledR}$ as a front end. MDI is a Mathematica package that defines a basic set of MFEDB access functions. The package will also accept standard SQL queries. Each function returns Mathematica-style lists, which can then be manipulated with any of the Mathematica functions. MDI also provides some utility functions for plotting and analyzing the data. The MDI package essentially makes the MFEDB an extension of Mathematica. The user may use any of the many Mathematica front-ends including telnet, $\mathrm{X}$-Windows, or a notebook. The mdi.m package may be obtained by anonymous FTP from the MFEDB site or by use of netmfe, an E-mail database interface.

MDI is a example of distributed computing. Behind the user interface, MDI calls an RPC client program that communicates with an RPC server on the MFEDB computer. It relies on the network communication capabilities of Mathematica to connect the user to a workstation running the Mathematica kernel. The Mathematica kernel is then connected to the MFEDB host workstation by a client/server pair of RPC processes. If the Mathematica kernel is to be run on the user's machine, the RPC client program must also be obtained and installed.

The MDI RPC server is also available for users who would like to provide their own client software. The server returns ASCII tables from standard queries and may be accessed and processed by any program on the internet that has access to RPC services.
\end{abstract}




\section{Introduction}

This report serves two functions. It provides an introduction and user's manual to MDI, and it describes in detail the underlying software, which may provide a useful example for others developing similar distributed applications. The MDI package is useful in its own right as it allows users to apply the powerful set of Mathematica ${ }^{0}$ operations directly to the data in the MFEDB ${ }^{1}$ database. The combination of MDI and Mathematica allows users to view, extract, and manipulate the MFEDB without having to understand either the SQL 2 query language or some of the more subtle interrelationships of the underlying relational database. The MDI package does allow more experienced users to present any SQL query to the database from within a Mathematica interface.

This report also describes the MDI RPC ${ }^{3}$ daemon, which provides network access to the MFEDB. Users may develop their own client programs to extract data from the database without using either the MDI client program, MDIClient, or the Mathematica interface. In this case the MDIClient program serves as an example.

Why MDI? Prior to the development of MDI, there were two methods of accessing the MFEDB. The most direct method required that the user obtain an account on the MFEDB host computer and use Oracle's SQL*PLUS ${ }^{4} 4$ query program. Once data had been extracted into a local file it was then usually sent by FTP5 to the user's host machine for further processing. This method is suitable for those users with good network access to the MFEDB host machine and who are familiar with the SQL query language. It is not particularly convenient for a cursory examination of the data or for casual users.

A second access method was modeled after the netlib ${ }^{6}$ software. Netlib is a program that accepts E-mail requests for numerical-analysis software or other information about the numerical-analysis community and sends back by return E-mail an answer, which may be a program or other text file. The netmfed $b^{7}$ service operates in a similar manner. It accepts a fixed set of requests by E-mail and responds by return E-mail. The commands accepted are of three types: requests for particular files, requests to execute predefined queries, and requests to execute a gineral SQL query. The netmfedb program solved three problems with MFEDB access. Firstly, the user no longer needed to have an account on the host machine. The netmfedb accepts requests from anyone who has access to Internet E-mail. Secondly, it expanded the potential users to those who have E-mail but not high-quality network access to the MFEDB host. Finally, the netmfedb program allowed certain queries to be packaged so that the user can request information without having to generate an SQL query or understand in detail the interrelationships between tables.

MDI provides additional capabilities beyond those provided by either SQL*PLUS or netmfedb and has different network access requirements. The MDI Mathematica package 
adds commands to Mathematica to access the MFEDB and to transfer and manipulate MFEDB data within a Mathematica environment. The powerful Mathematica listprocessing, computational, and graphics capabilities can be directly applied to the MFEDB. As part of the MDI system, an RPC service has been established on the MFEDB host computer. This service provides MFEDB access to programs that have RPC network access but do not require Mathematica.

In order to use the Mathematica package, the user obviously must have access to Mathematica. There are two ways this can be accomplished. Users with a Mathematica front end installed can simply use the Mathematica network connection tools to connect to the Mathematica kernel running on a workstation at the Fusion Research Center. A special account has been set up for this purpose.

Users who would like to run the Mathematica kernel on their own machines or to access the MFEDB from within their own programs have somewhat different requirements. The user's host machine must have RPC access to the MFEDB host machine, but the user is not required to obtain an account on the MFEDB host. The MDI user must also be able to install either the MDIClient or a similar RPC client program on his host computer. For MDIClient, this requires a $\mathrm{C}$ compiler and an RPC library. These should $\mathrm{b}_{c}$ available on most Unix machines and on VMS machines equipped with Multinet ${ }^{\circledR}$ or equivalent TCP/IP8 software.

The remainder of this report is organized as follows: Section II describes the Mathematica commands that MDI defines and serves as a user's guide to MDI. Section III presents some examples of huw the MDI commands can be combined with standard Mathematica commands to generate more powerful queries and reports. It is the combination of Mathematica and MFEDB that allows very powerful data access and rnanipulation commands to be constructed. Section IV describes the client/server RPC model and describes the Mathematica-MDIClient interaction. Section V describes the MDI RPC server. Appendix I contains a diagram of the MFEDB. Appendix II is a listing of the MDIClient code and Appendix III is a listing of the MDI RPC server software. The MDI package is listed in Appendix IV.

\section{MDI commands}

MDI is a Mathematica package, mdi.m, which must first be loaded into the Mathematica environment. For users running the kernel on the Fusion Research Center workstation, this can be accomplished with the command $<<$ LOcal ' mdi `. Each of the MDI commands begins with the prefix "Mdi". A complete list of commands can be found after loading the MDI package by executing the Mathematica command ?Mdi *. A brief 
description of each command may be found by using the Mathematica ? operator. Most MDI commands return either a list of strings or a graphic object. The strings may need to be converted into numbers for certain Mathematica operations. The function MdiToNumber is provided for this operation. The MdiProfile and MdiProfilexY commands convert the lists into numbers directly.

\section{A. MFEDB}

To make use of the MFEDB one should be familiar with some of the terms used in the database and the general organizational structure of the database. Appendix I contains a diagram of the internal relational structure of the MFEDB and shows the important links between the tables. Use of the MDI command largely obviates the need to understand how these links are used or the organization of the database; however the MFEDB does define certain terminology that should be understood. Each experiment is referred to as a machine. For each machine the data is organized in terms of discharges or shots and these discharges are numbered with a shot number. Experimentalists often refer to particular discharges in terms of certain nominal parameters, even though these parameters may only be approximately obtained during part of the discharge. These parameters describe the discharge conditions and include the toroidal field, plasma current, average electron density, etc. Discharges are also often characterized by different terms such as the type of heating, ohmic, beam, etc., or the boundary conditions, limited, diverted; these terms are called keywords. Keywords are words that describe the discharge without needing an associated value. Other scalar characteristics of the discharge, such as particle confinement time require both a parameter name and value. These are called characteristics, and the value is the characteristic value.

The MFEDB is not an experimental archive and does not store raw data. All data has been analyzed and converted to physically meaningful quantities, such as electron temperature, electron density, etc. These physical quantities are usually measured at multiple points across a discharge at a given time to form a radial profile or are measured at a given position for multiple points in time to form a temporal profile. In general, quantities that have values associated with an independent variable are called profiles. Each profile is characterized by its name, the independent variable, either space or time, the coordinate system, how the data are stored etc. Each profile is stored either as a fit to a specified function or as data-point four-tuples, $(x, d x, y, d y)$, although the error values are often zero. Spatial profiles that have been taken at several times during a discharge are numbered by a slice number. Profiles that are functions of time, are given a slice number 
of -1 . The database also stores the name of the person or group responsible for each profile.

Many tokamak experiments consists of perturbations made to a plasma that is considered to have reached steady-state. Perturbations such as pellet injections, additional heating methods, etc., are characterized by anothier set of scalar parameters and their values, called perturbations.

Most of the database entries allow textual comments to be included. These comments have been organized as footnotes and are referenced by a footnote or comment number. These comment numbers, which are returned by several MDI commands, can then be used to retrieve the comments. Note that a comment number of 0 implies "no comment".

Many of the discharges in the database have appeared in published papers or reports. These references are stored in the database and each reference has been given a reference number. The references related to a particular machine can be found as can the discharges associated with a particular reference.

The machines or experimental devices are also described by sets of parameters and values which do not change during a shot and change only when the machine configuration changes. These parameters, which may describe the positions and types of limiters, for example, have two-part names. The parameters are grouped into sets called machine parameter sets. Within each set the parameters are called machine parameters. The database contains a dictionary of most of the words and special terms, which makes the database largely self-defining. Descriptions of coordinate systems and fitting functions are also contained in the dictionary.

Within the database, special variables or column names are used that correspond to the terms used in MDI; for example, a profile is a physparam, a characteristic parameter is a charparam, a perturbation is a pertparam, etc. It is not necessary to know these special names to use MDI; however, it is necessary to use these names in SQL queries.

\section{B. The commands}

The MDI commands are organized here according to the type of Mathematica object returned and ure also approximately in the order in which a new user might wish to explore the database. An alphabetical list of commands can be found in section VI. The MDI commands are highlighted with brief descriptions of their function. The command parameters are usually strings and are set off in double quotation marks. The shot numbers can be given either as strings or integers. The reference numbers and comment numbers should be given as integers. Some parameters are optional and are denoted as such by 
being followed by a colon and the default value of the argument. The colon should not be entered. Commands that end in "table" are designed to be used with the Tableform output format.

Many commands return a single Mathematica list. These lists can be combined with the Mathematica list-manipulation commands and other MDI commands to easily generate more complicated queries. Combining these commands with list operations is demonstrated in section III.

A list of all the machines represented in the database can be found with the Mdimachines command.

MdiMachines [] returns a list of machines in the database.

$\operatorname{In}[2]:=$ MdiMachines []

Out $[2]=$ (ASDEX, JET, JT-60, PDX, PLT, T-10, TEXT, TFTR )

The published references associated with a particular machine can be found with the MdiReferences command.

Mdi References [ machine"] returns a list of reference numbers and the references associated with a particular machine.

Several commands return a list of shot numbers that can then be used as input for other MDI commands.

Mdidischarges [ "machine"] returns a list of shot numbers for given machine.

MdiDischargesFul1 ["machine"] returns a table of shot numbers for given machine including the characterization data for each discharge.

MdiDischargesByType [ machine", "keyword"] returns a list of all shot numbers for a machine that are described by the same keyword.

MdiDischargesByReference [ "reference number"] returns a list of machine names and shot numbers associated with a particular reference. 
For example, to find all ohmic shot numbers for TEXT the command MdiDischargesByType [ TEXT", "ohmic"] could be used.

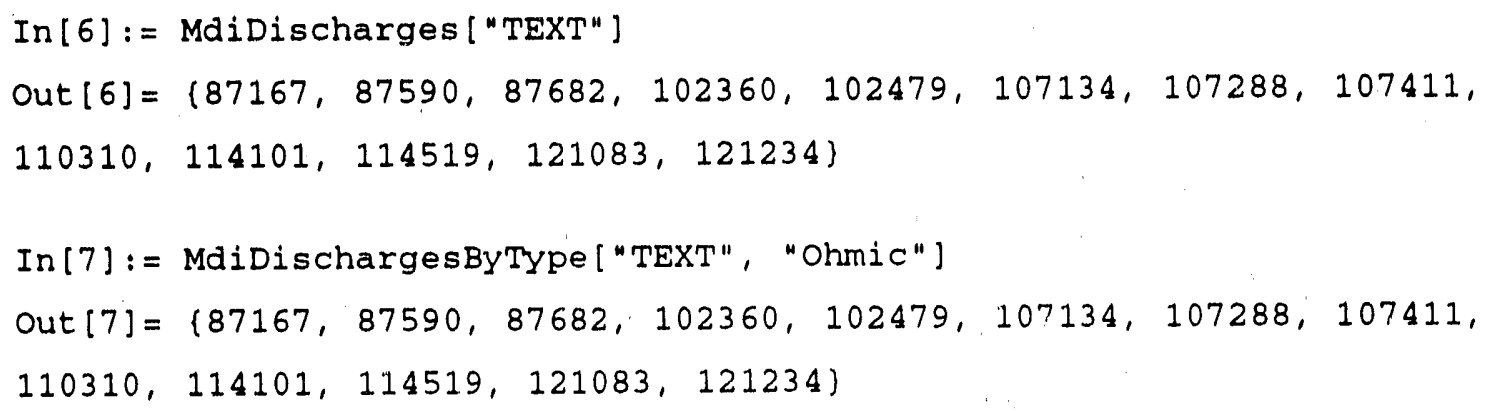

The keywords for a discharge can be found with the Mdikeywords command.

Mdikeywords ["machine", shot number] returns a list of keywords describing the discharge.

Mdikeywords [ machine"] returns a list of all unique keywords for the machine.

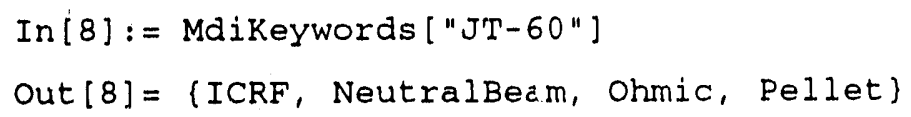

The keywords are used to broadly classify discharges in types such as diverted, or limited etc. Discharges are also typically characterized by certain parameters.

Mdidischargeconditions ["machine", shot number] returns the nominal Bt, Ip, NeBar, VLoop, Gas, Zeff, number of time slices for a given discharge, and the comment number in the indicated order.

For example,

In[9]:= MdiDischargeConditions ["TEXT", 121234]

Out $[9]=\{2,200000,3.0000 E+19,1.518,1,1.636,1,0\}$

which denotes a $2 \mathrm{~T}$ magnetic field, $200 \mathrm{kA}$ plasma current, etc. discharge. Note that the data in the database is generally in MKS units. For a tabular result which includes the heading see the MdiDischargeconditionsTable command.

The machines and discharges are described by additional parameters as well. For example there are parameters which describe the machine configuration, i.e., limiter position and composition. 
MaiMachineparameters ( "machine"] returns a list of the names of all the machine parameter sets for the machine.

Mdimachineparameters [ "machine", shot number] returns a list of the names of machine parameter sets and machine parameters and their values for this shot.

Maimachinevaluel "machine", shot number, "machine parameter set", "machine parameter"] returns the value of a particular machine parameter.

For example, a partial listing of the result of TableForm [MdiMachineParameters ["TEXT", 121234]],

$\begin{array}{llll}\text { gettered } & \mathrm{N} & 0 & 0 \\ \text { lastvent } & 20 \text {-feb-1988 } & 0 & 0 \\ \text { limiter } & \text { TiC } & 0 & 0 \\ \text { limiter } & \text { phi } & 294.5 & 0 \\ \text { limiter } & \text { rmajor } & 1 & 0 \\ \text { limiter } & \text { rminor } & .26 & 0,\end{array}$

shows that this discharge was not gettered, when the machine was last vented, and the position, composition, and size of the limiter. The last column displays the comment number-here indicating no comments were entered.

Discharges are also described by their major and minor radius, the particle and energy confinement times, etc. These parameters are called characteristics and can be accessed with several MDI commands.

Mdicharacteristics [ machine"] returns a list of all unique characteristic names for the machine.

Mdicharacteristics ["machine", shot number] returns a list of characteristicc names for the discharge.

MdicharacteristicsFul] [ machine", shot number] returns alist of characteristic names for the discharge and their values.

Mdicharacteristicvalue [ machine", shotnumber,

"characteristic"] returns the value of characteristic name for this discharge. 
Malcharacterist icslist I machine", shot number,

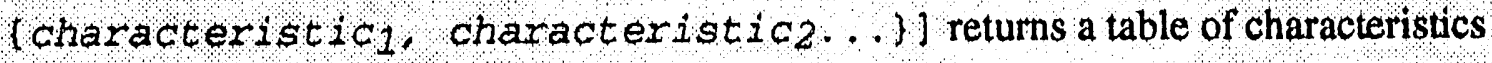
and their values.

For example,

In[10]:= MdiCharacteristics["TEXT", 121234]

out $[10]=$ (rmajor, rminor, taue, tauee, taup

Discharges are also characterized by the perturbations applied during the discharge.

Mdiperturbations [machine", shot number] returns a list of perturbation parameters and their values.

For example, TableFórm [Mdiperturbations ["TEXT", 121234]] returns:

$\begin{array}{llll}\text { DNB } & \text { DNBCur } & 4.7 & 0 \\ \text { DNB } & \text { DNBPulse } & .08 & 0 \\ \text { DNB } & \text { DNBTime } & .31 & 0 \\ \text { DNB } & \text { DNBVolts } & 44000 & 0,\end{array}$

which describes the diagnostic neutral beam for this discharge. The last column is the comment number.

The dictionary can be used to lookup any term found in the database.

Maldefinit ion [ word"] returns the definition of word as a string.

For example, MdiDefinition ["TeTs"] returns:

TeTS - Electron temperature (keV) measured by ThomsScat.

The dictionary can be searched for words which match certain patterns.

Mdidictionary [ pattern"] returns list of words that match the pattern and have definitions in the dictionary.

The wild card character is the "\%" symbol. For example to find all words beginning with rx:

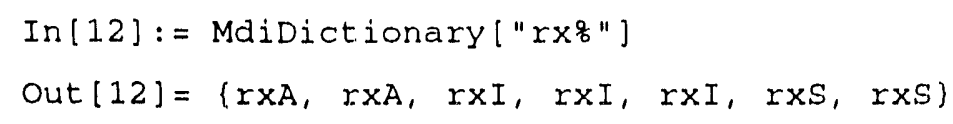


Several of the commands return comment numbers. Mdicomment is used to retrieve the comment.

Mdicomment [comment number] returns the comment referred to by comment number as a string.

The commands in the nexi group return formatted tables or reports. They are most often used simply for their results rather than as input to other commands. Note that the database management system, DBMS, generally returns a table complete with column headings. These headings are usually removed by the MDI commands, but the headings from the last query or command can be recovered with the MdiHeadings command.

MdiDischargestable [ "machine"] returns a table of shot nu nbers with characterization data including column headings.

MaidischargeConditionstable ["machine", shot number] returns a table of discharge characteristics with headings for a particular shot.

Mai Perturbationstable [ machine", shot number] returns a formatted table with headings of the perturbation information.

The primary purpose of the MFEDB is to store profile data, i.e., radial profiles of important tokamak parameters such as the electron temperature. Commands in the next group are used to retrieve and plot profile data. Commands that require a machine name and shot number usually also need a slice number. If a discharge has radial profiles at more than one time, then these profiles are indexed by slice number. Each of these will default to slice $=1$, so that the last argument can be omitted if only the first slice is required. Note that profiles that are functions of time have a slice number of -1 , so that these functions must have the slice number specified.

Each profile has been given a unique name. The MdiProfiles command returns the names of all profiles stored for this discharge.

Mdiprofiles [ machine", shot number] returns a list of profile names for this discharge. 
The MdiprofileData command returns the name of the independent variable which specifies a particular coordinate system. A description of the coordinate system can be found in the dictionary. The name of the fixed variable and its value is returned. If the profile is stored as a functional fit, the name of the fitting function is given. A comment number is also returned.

Malprofiledata ["machine", shot number, "profile", slice:1] returns information about the profile.

Information about the person or group responsible for a given profile can be found with maisource command.

Maisource ["machine", shot number, "profile"] retuins information about the person or group responsible for this measuremient.

The main command for retrieving profile data is the Mdiprofile command. The commands MdiProfile and MdiProfilexy return matrices of numbers rather than a list of strings. These matrices can be used directly in Mathematica numerical operations and plotting functions.

Mdiprofile["machine", shot number, "profile", slice: 1$]$ returns a list of $\mathrm{x}, \mathrm{dx}, \mathrm{y}, \mathrm{dy}$ four-tuples.

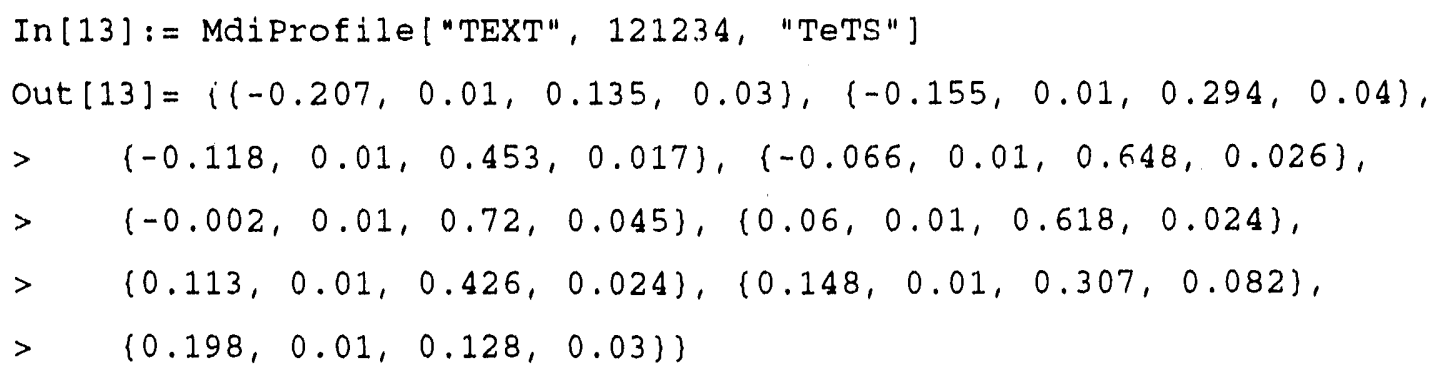

In the cases in which error values have not been provided or only the $x-y$ pairs are required, then the MdiProfilexy command can be used.

Mdiprofilexy ["machine", shot number, "profile", s1ice:1] returns the a list of $x-y$ pairs. 
The list can be used directly in the ListPlot command. For example

ListPlot[ MdiProfileXY["TEXT", 121234, "TeTS"], PlotJoined->True, PlotRange->All];

generates a plot of the electron temperature:

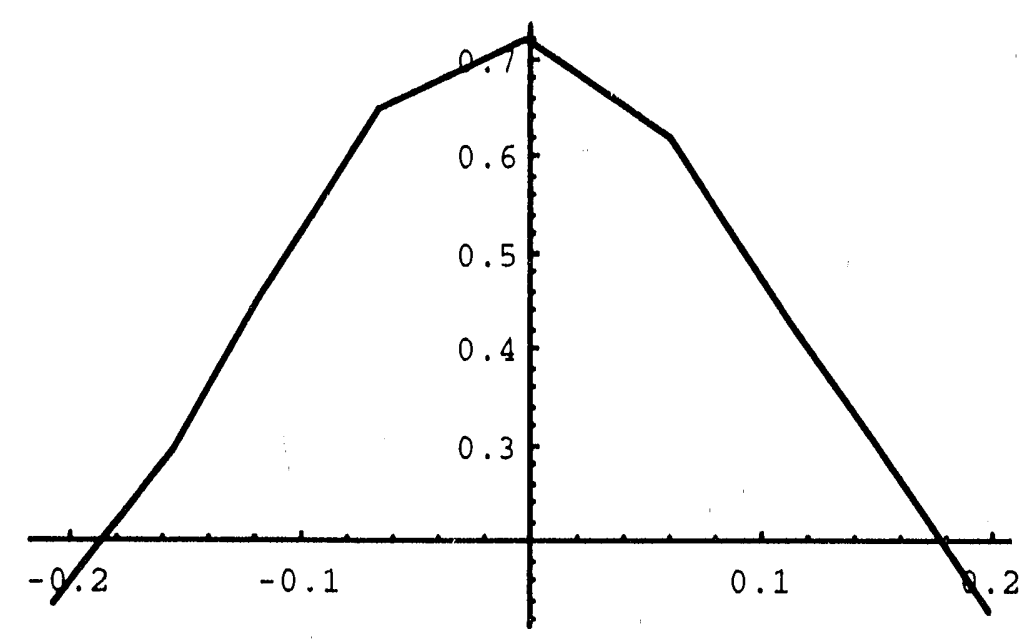

Mdiprofllesizel "machine", shot number, "profile", slice:1]

returns the number of points in a profile.

Profiles can be plotted with the axes appropriately labeled with the MdiProfilePlot command.

Mdiprofileplot ["machine", shot number, "profile", slice:1] returns a graphic object including error bars if available.

In[16]: = Show [MdiProfilePlot ["TEXT", 121234, "TeTS"]] 


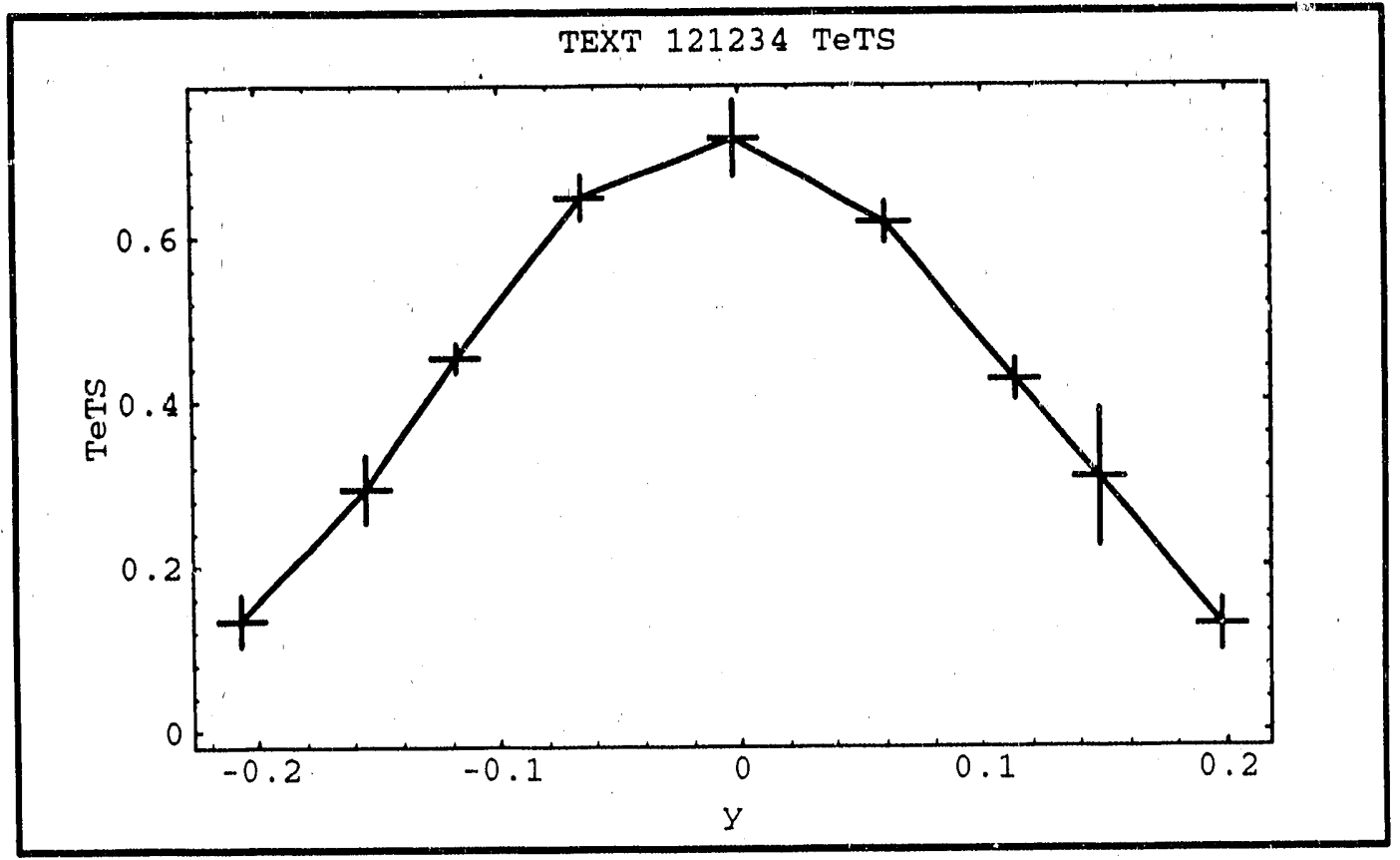

Note that graphics objects rather than plots are returned. Show can be used on the result, or else the command can be an argument of Show.

Some profiles are described as functions with their function fit parameters stored in the database. These parameters can be retrieved with the Mdiprofilefit command. The description of the function is stored in the dictionary. The name of the function can be found with the MdiProfileData command.

Maiprofilefit["machine", shot number, "profile", slice:1] returns the fit parameters.

There are several profiles that are generic to tokamak data and which are necessary for transport analysis. These have their own plot commands so that differences in the profile names and dimensions among the different machines can be ignored. Each of these functions simply calls the MdiProfilePlot command with the appropriate profile name for the given machine.

Mdireplot [ "machine", shot number, slice:1] returns the generic electron temperature profile. 
Md'riplot l "machine", shot number, slice:1] returns the generic ion temperature profile.

MdiNeplot I "machine", shot number, slice: 1] retums the generic electron density profile.

Malpohplot [ machine", shot number, slice: 1$]$ returns the generic ohmic power profile.

Mdipauxplot ["machine", shot number, slice:1] returns the generic auxiliary power profile.

A single page summary of the discharge can be obtained with the command, Mdisummary, which collects the nominal parameters and the slice 1 generic profiles.

Maisummary [ "machine", shot number] returns a graphic array of plots for Te, Ti, ne, Poh, Paux and a table of the nominal discharge parameters.

For generating profile plots within Mathematica it is sometimes useful to have the name of the abscissa.

Mdiprofllextabel ["machine", shot number, "profile"] returns the name of independent variable for the profile.

The Mathematica function, Interpolation, returns a function that can be evaluated anywhere within the range of the supplied data. It is used to construct the next set of commands.

MdiprofileIf ["machine", shot number, "profile", slice:1] returns an interpolation function for a profile.

There are special interpolating functions for each of the generic functions.

MdiTeIF["machine", shot number, slice:1] returns the interpolating function for generic electron temperature profile. 
MaITIIF [ machine", shot number, s11ce. I] returns the interpolating function for generic ion temperature profile.

MdiNeIf [ machine", shot number, si ice.1] returns the interpolating function for generic electron density profile.

MalPohIF [ machine", shot number, s 1 ice: 1 ] returns the interpolating function for generic ohmic power profile.

Mal pauxTF[ machine", shot number, slice: 1$]$ returns the interpolating function for generic auxiliary power profile.

MDI is not limited to queries that have been preprogranmed either in the MDI package or in the RPC server. Any SQL query can be sent to the database. The query should be a single string and should not end in a semicolon. The RPC transport limits responses to about $200 \mathrm{~K}$ bytes, so queries should be formulated to keep the query result table less than this amount.

Mailouery [ ouery...] submits the query given as a text string to the database and returns the resulting table in Mathematica list form.

For example,

In[17]:= Mdiquery["select shotnum, ip from discharges where machine ='TEXT' and ip > 290000"]

Out $[17]=\{\{(107411\},\{300000\}\},\{\{107288\},\{300000\}\},\{\{107134\}$, $\{300000\}\},\{\{102479\},\{300000\}\},\{\{102360\},\{300000\}\},\{\{87682\}$, $\{300000\}),\{(87590\},\{300000\}\})$.

Some retrieval problems which would require many separate queries when done with the Mathematica list-processing commands can be done with a single SQL query. In these cases while the MDI commands may be more convenient, the SQL query would be more efficient. The MDI package allows the user to combine both approaches.

If the MDI command returns an error message or a surprising result, it is sometimes useful to examine in more detail the results of the previous command. The following commands provide additional information.

MdiLastQuery [] returns the exact form of the last query submitted. 
MalRows 1 returns the number of rows in the last query result table.

Mdi Headings [] retums the DBMS table headings from the last command.

Mai Er rominessage (1) If the last query generated an error message from the database engine or from the RPC commuruication layer, this command can be used to retrieve the full text of the message.

MaLRawReturn I retums the unprocessed result from the MDIClient program.

Mdi Command $]$ returns the command line of the last command submitted to the shell.

The output from MDI can be exported to other programs or sent to various output devices in the same way as other Mathematica results. There are a few additional commands that simplify some common output operations.

Mal Tospreadsheet $"$ fl l ename", table name] writes a data table to an external file in a form suitable for a Macintosh spreadsheet program.

The file will be created in the current directory. The fields are tab delimited, the records are carriage-return delimited, and numbers are converted to CForm.

MdiPSPrint [ -graphic- ] sends postscript graphics to a particular Apple Laser Writer printer.

The MdiPSPrint command should be modified for each installation.

The Mdiprofiletable command was constructed to solve a particular database access problem. A listing of the Mathematica program for this command can be found in Appendix IV and can be used as a example program for solving data retrieval problems that cannot be done with a single MDI command.

Maiprofiletable [ machine", [shot number list], (profile list]) returns a table of profile names for each shot on the list in which each has the same radial points. 


\section{Examples}

The MDI commands provide access to most information in the MFEDB. The true usefulness of MDI is in the combination of MDI commands with the Mathematica extensive list and numerical commands. The examples in this section show some typical uses of MDI.

The MdiSummary command produces a report for a particular discharge. To generate a series of reports for a machine, the following function could be used:

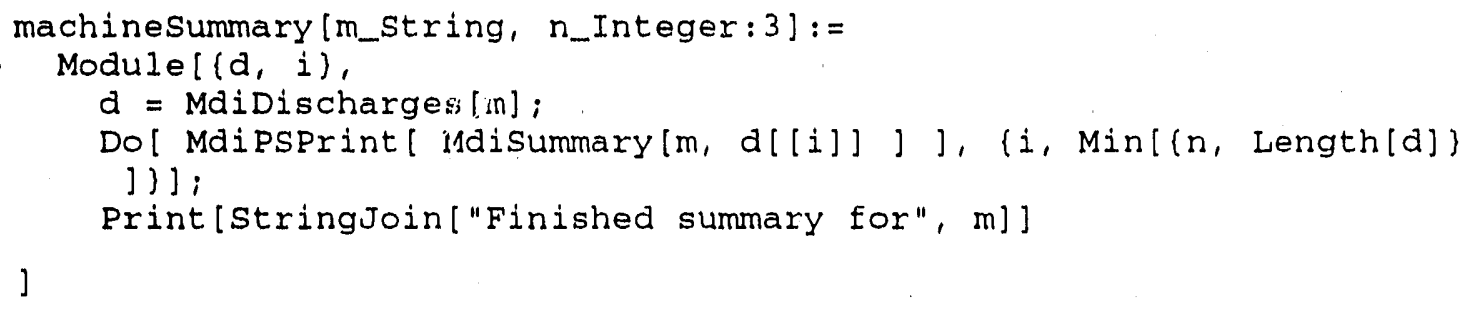

If there are 1000 shots for a machine then this command could generate up to 16,000 queries and produce a 1000 page report. The default number is set at 3 . The list processing is powerful and should be used with some care.

The interpolation functions can be used to construct other functions, which can then be plotted or operated on by any of the Mathematica special functions. For example, the electron thermal velocity function for a discharge can be constructed by:

te $=$ MdiTeIF["JT-60", 7849];

vte $\left[x_{-}\right]:=4.1910^{\wedge} 7$ Sqrt $\left[10^{\wedge} 3\right.$ te $\left[x_{]}\right]$]

The function vte $(x)$ can now be evaluated anywhere within the range of data for that shot and can be used in other Mathematica operations.

To plot one nominal characterization parameter against another the following function could be used:

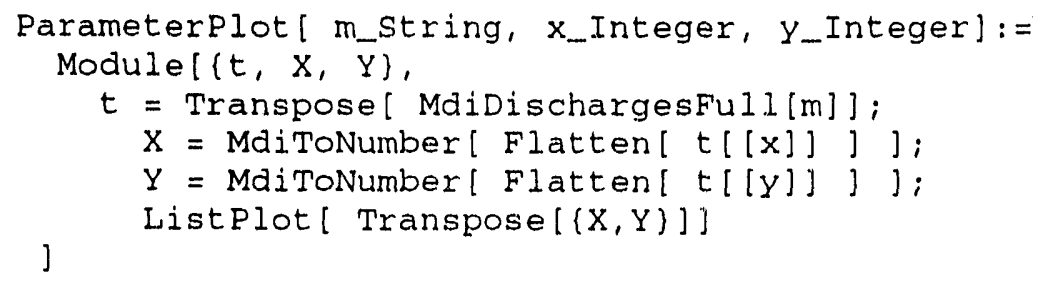

Here $\mathrm{x}$ and $\mathrm{y}$ are the column numbers in the table returned by MdiDischargesFull. 
To make a table of all key words for all machines in the database, the following command could be used:

Map [(\#, MdiKeywords[\#]\}\&, MdiMachines[]]//TableForm.

To make a table of the number of slices for each discharge for a machine, define:

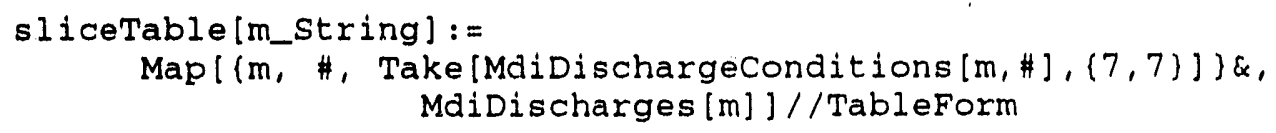

To find the number of shots of a certain type and machine, define:

Typedshots[m_string, t_string]: =

$(m, t$, Length [MdiDischargesByType $[m, t]])$

Then to build a table for all machines of that type, use

Map[ TypedShots[\#, "Ohmic"]\&, MdiMachines[]]//TableForm.

If one wanted to find all shots in a given reference with a particular type, one could use the command:

Intersect ion [Flatten [Drop [Transpose [MdiDischargesByReference [2]], 1]], MdiDischargesByType ["TFTR", "ohmic"]] .

Suppose one wanted to generate a Murakami plot of the TFTR ohmic discharges in the database. The Murakami parameter is $\mathrm{n}_{\mathrm{e}} \mathrm{R} / \mathrm{Bt}$, and the Murakami plot is the energy confinement time plotted as a function of the Murakami parameter. We first get a list of shots:

shots = MdiDischargesByType["TFTR", "Ohmic"];

Then we make lists of the major radius, confinement time, and other characteristic parameters:

$R=$ MdiToNumber [Map [MdiCharacteristicValue["TFTR", \#, "rmajor"]\&, shots]];

tau = MdiToNumber[Map[MdiCharacteristicValue[ "TFTR", \#, "TAUE_A_TOI\$R"]\&, shots ] ];

(Bt, Ip, Nebar, V1, Gas, Zeff, Ts, Cid\}=

Transpose [MdiToNumber [Map [MdiDischargeConditions ["TFTR", \#] \&, shots]]];

We next calculate the Murakami parameter for all the shots:

Mur = Nebar R /( Bt 10^19);

then we make a list of $\mathrm{x}, \mathrm{y}$ pairs in a form suitable for ListPlot: 
pts $=\operatorname{Transpose}[\{$ Flatten[Mur], Flatten[tau $]\}] ;$

There is one problem because some shots did not have a confinement time available. These shots have EndOfFile as their $y$ value. We simply select those points without this property:

npts = Select [pts, \#[12]]!= "EndofFile"\&];

We are now ready to make the plot:

ListPlot [npts, Frame->True, FrameLabel-> ("Murakami", "tauE", " ", " ")];

with the result:

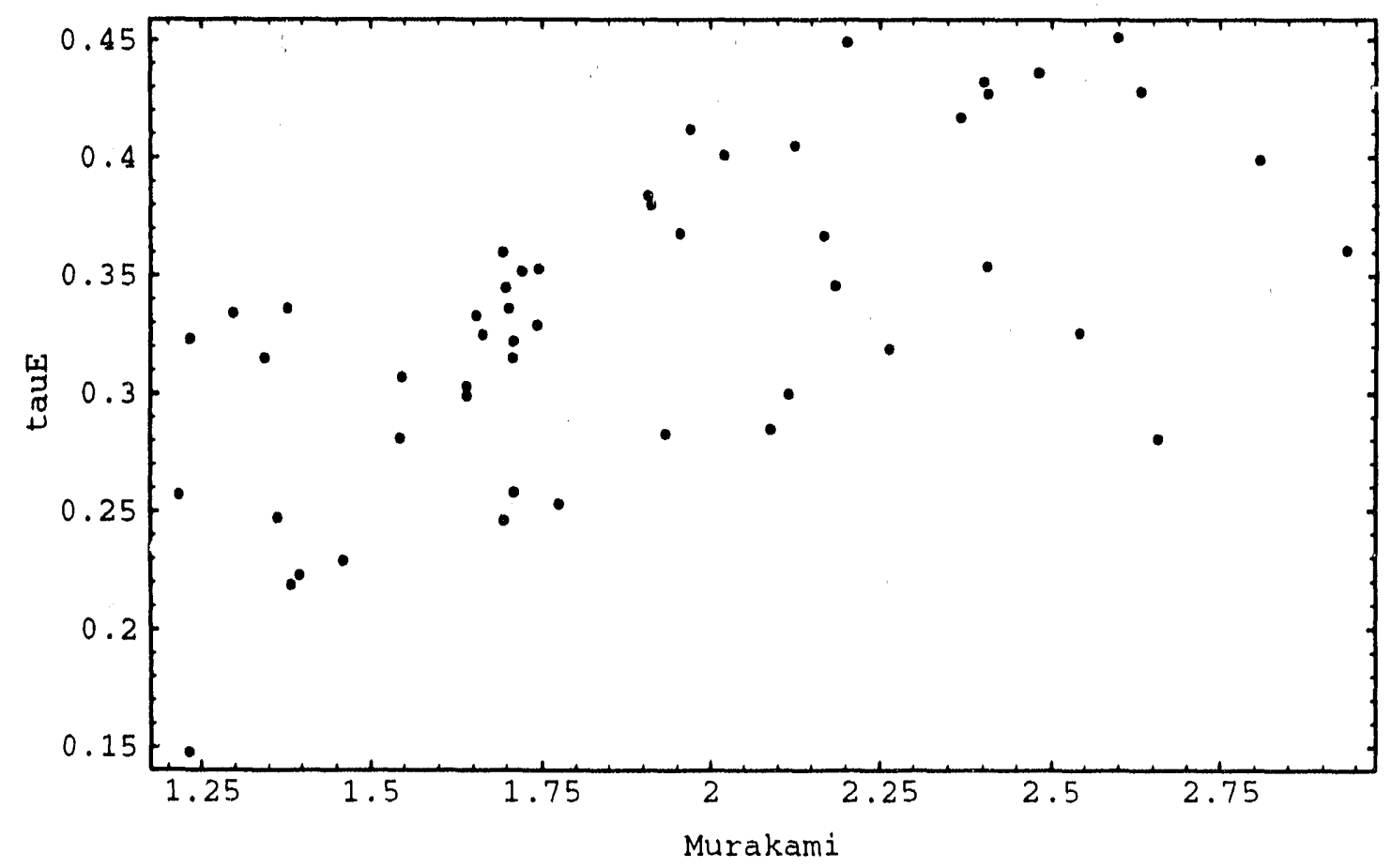




\section{The MDI components}

The MDI system is composed of three parts: the Mdi.m package, which adds the database access functions to Mathematica, the RPC clien//server process pair, which provide network communication between the Mathematica kernel and the MFEDB host computer, and the Oracle database manager software, which handles the MFEDB. Figure 1 shows a schematic layout of the MDI components. There are potentially three computers involved and five separate processes. The network connection between a Mathematica front-end and the Mathematica kernel is controlled by the Mathematica software. These two processes can either run on the same computer or be connected through a network or by modem.

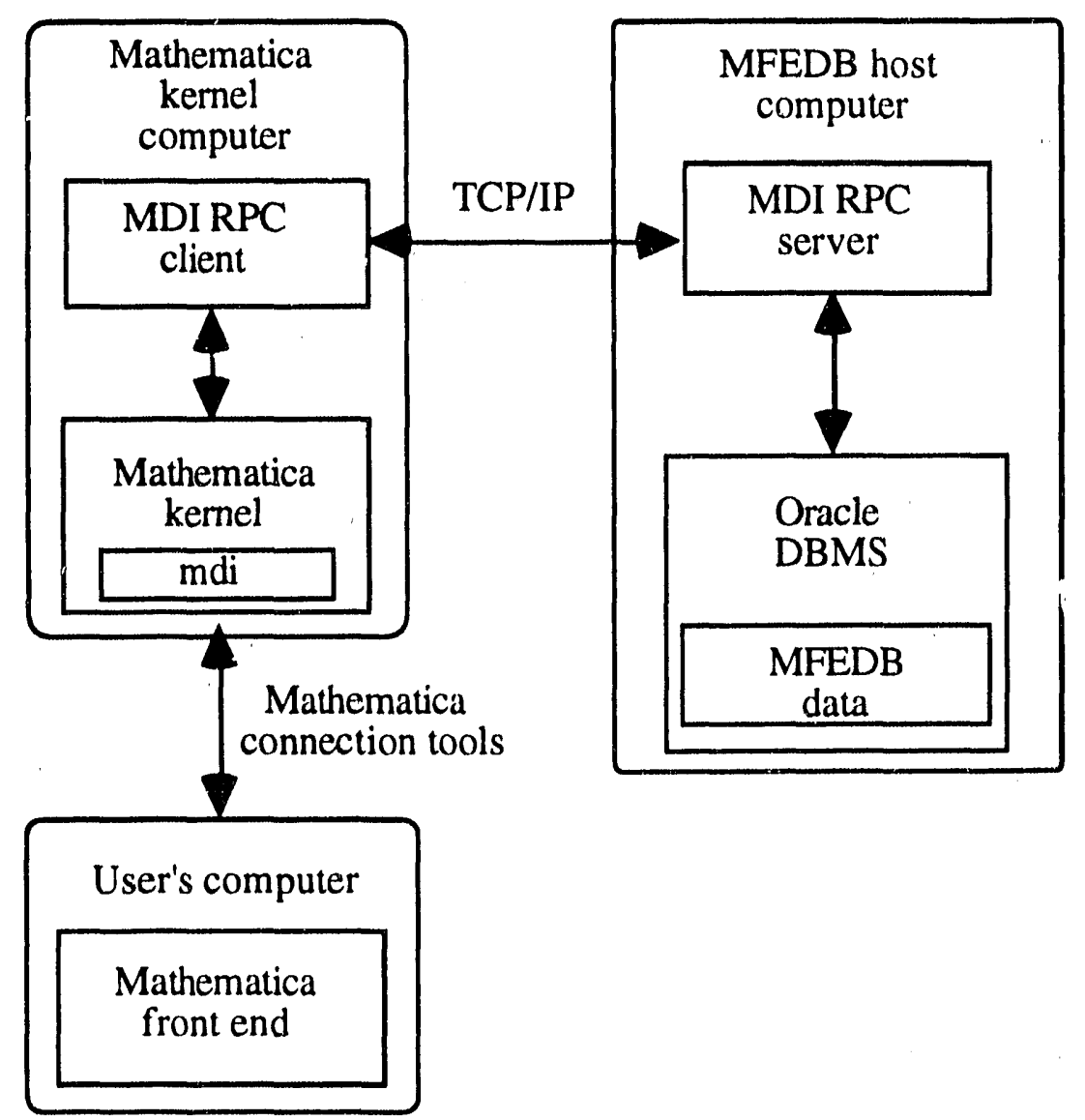

Figure 1. The MDI components.

There are three inter-process communication connections in addition to the Mathematica kernel-front-end connection. Two of these connections are between processes running on the same computer, and one connects processes running on different 
machines. When data are transmitted between processes there is always the problem of maintaining compatible formats, éspecially when processes may reside on different computer architectures with different internal data representations. The RPC protocol was designed to solve this problem by including a description of the argument data types in the argument list and defining, through the XDR protocol, standard network data formats. The MDI package largely avoids these problems by exchanging data between processes as text streams.

A second design consideration in multi-process applications is process synchronization. The MDI package is designed as a single execution pipeline, i.e., each process initiates the next process in the sequence and blocks until it either receives a result or times out. Once a process receives a result from the next process in the pipeline it completes its processing and terminates. The chain of process is re-created with each call to an MDI function. An alternate design would require that the MDI package have an open command that would establish the chain of processes to the database manager. MDI commands could then be executed with each of the processes already established, saving the start-up overhead. There would then need to be a close command, which would release each of the processes in the chain. While this design may result in somewhat faster queries, it is significantly more complex and would require more sophisticated programming and error correction at each stage. Simple MDI commands, such as Maimachines execute in about one second, which provides a good estimate of the amount of time required to set up the process chain. More complex queries take longer, but most of this time appears to be due to the database manager and the Mathematica processing of the results. For simple applications like MDI the current design appears to be adequate. It can also be viewed as an application prototype with more sophisticated inter-process communication methods added as necessary.

On most computer systems, with the Macintosh being the most notable exception, Mathematica can execute an external program from within the Mathematica session. There are two types of communication with the external process: structured and unstructured. The structured communication uses the MathLink protocol and allows programs to exchange Mathematica expressions of all types. The unstructured communication simply transmits text streams using file read and write operations.

The MDI package on the Mathematica kernel computer communicates with the MDI client program using the Mathematica function, Get. The Get function performs unstructured communication with an external program. The argument to the Get function contains the complete shell command line to run the MDI client program. This command contains the name of the remote MDI server machine, the integer code of the function to be 
performed and a single text string containing the argument to the function. The output of this program, which is written to stdout, is piped to Mathematica as if Mathematica were reading a text file. Because all data is transmitted as text strings, there are no problems with data format or internal data representations. The conversion between numbers and text is done in MDI.

The MDI client program, MDIClient, is the network glue that connects the Mathematica kernel computer to the MDI RPC service. A listing of MDIClient is provided in Appendix II. It performs three functions: checks input parameters, calls the MDI service, and formats the result as Mathematica lists. Of the three levels of RPC library routines, MDI uses the lowest level so that the communication protocol can be chosen and the time-out intervals can be set. The TCP rather than the UDP protocol is used to obtain a guaranteed once-delivery message service. The problem with the default UDP protocol is that the database manager may require more time to complete the query than the UDP protocol allows. In this case, the database manager would continue to receive additional requests, each of which would take longer than the allowed time-out interval. The MDI query would then eventually fail even though the query could be performed in the total time-out period allowed. By using the $1 \mathrm{CP}$ protocol, the database manager is guaranteed to receive the message only once and the time-out period can be controlled. A time-out value of about 90 seconds is typically used. Most queries complete in less than a few seconds so 90 seconds is generally more than adequate. Since MDI is often used in the interactive mode, the time-out period must be long enough to complete the query, but short enough not to waste time if the query or network connection fails.

The MDIClient program can be used as an example for the development of other client programs that communicate with the MDI server. At this level there is no requirement for Mathematica. Any program that can make RPC calls can use the MDI service.

The MDIClient program can be executed from the Unix command line. Mathematica is not needed. For example, \$MDIClient hagar.ph.utexas.edu 2 "TeTS"

executed on a machine with MDIClient installed would return the definition of TrTs enclosed in braces. 


\section{The Mdi RPC server}

The MDI RPC server is an RPC service installed on the MFEDB host computer that responds to a fixed set of commands. All commands have the form of an integer followed by a text string. The integer codes the particular function that the service is to perform. The arguments to that function are contained in the text string. Each function builds and submits a query to the MFEDB. The database manager returns an ASCII coded table. The table is converted into a single text string with embedded carriage-returns as record delimiters. This string is returned as the RPC function return.

The commands are grouped according to the type of their arguments. The constants used to denote each routine are given names here, but these names are only informative. These commands represent a minimum set of SQL queries needed to access most of the data in the database. Note that the terminology used here corresponds to the database internal-column names and not that of $\mathrm{MDI}$, i.e., physparam not profile etc.

Functions with text string as the argument: manager.

1. kSQL - The text string contains the rull query which is presented to the database

Functions with a single word as an argument:

2. kDefine - The string contains a word to be looked up in the dictionary, A single string containing the definition is returned.

Functions with no arguments:

3. kMachines - A list of machines in the database is returned.

Functions with the machine name as argument:

4. kDischarges.- A list of shot numbers is returned.

5. kDischargesFull - A table of discharges along with the nominal characterization data is returned.

19. kReferences - A list of all references for this machine is returned.

27. kCharparamsAll - A list of all charparam names for this machine is returned.

24. kKeywordsAll - A list of all keywords for this machine is returned.

25. kMachineAll - A table of the names of all machine parameters and machine parameters for this machine is returned.

Function with the machine name and keyword as argument:

6. kDischargesTyped - A list of shot numbers is returned.

Functions with the machine name and shot number as arguments:

17. kPhysparams - A list of physparams for this discharge is returned.

18. kCharparamFull - A list of charparams and values is returned. 

retumed. retumed.

7. kCharparam. - A list of charparams for this discharge is returned.

8. kNominal - The nominal discharge characterization parameters are returned.

9. kKeywords - A table of keywords and values which describe the discharge is

10. kPerturbations - A table of perturbation information for the discharge is

29. kMachineParam - A tahle of machine parameter sets, machine parameter names and their values for this shot is returned.

Functions with machine name, shot number, and charparam as arguments: returned.

11. KCharparam Val - The value of the particular charparam for this discharge is

Functions with machine name, shot number and list of charparams as parameters:

12. kCharparamList - A list of values corresponding to the list of charparams for the given discharge is returned.

Functions with machine name, shot number, physparam, and slice number as arguments:

13. kPhysparam - A four column table with $\mathrm{x}, \mathrm{dx}, \mathrm{y}$, dy data is returned.

14. $\mathrm{kPhy}$ sparam $X Y$ - A two column table with $x, y$ data is returned.

15. kPhysparamSize - The number of points in the physparam is returned.

16. kPhysparamXLabel - The name of the abscissa is returned.

22. kPhysparamData - Information about the physparam is returned.

28. kPhysparamFit - Table of fit parameters for this physparam is returned.

Functions with machine name, shot number, and physparam as arguments:

21. kSourre - The person are group responsible for the measurement is returned

Functions with 'omment number as an argument:

23. $\mathrm{kC}$ mment - The comment associated with the comment number is retumed.

Functions with reference number as argument:

20. kDischargesByRef - A list of shot numbers and machine names associated with the reference is returned.

Functions with machine name and machine parameter as agruments:

26. kMachine Val - The value of the machine parameter is returned.

A comparsion of the functions provided by this RPC service and the tables in the database as given in Appendix I shows that all the data can be accessed. 
The MDI server code communicates with the Oracle database by building a text file containing the necessary "set" commands and the query. This file is used as an input file for the SQL*PLUS program, which is executed using the Unix "system" command. The output is written into a temporary file that is then processed by the MDI server program and returned to the MDI client program.

A complete listing of the MDI server code is giverı in Appendix III.

\section{DISCLAIMER}

This report was prepared as an account of work sponsored by an agency of the United States Government. Neither the United States Government nor any agency thereof, nor any of their employees, makes any warranty, express or implied, or assumes any legal liability or responsibility for the accuracy, completeness, or usefulness of any information, apparatus, product, or prociss disclosed, or represents that its use would not infringe privately owned rights. Reference hercin to any specific commercial product, process, or service by trade name, trademark, manufacturer, or otherwise does not necessarily constitute or imply its endorsement, recommendation, or favoring by the United States Government or any agency thereof. The views and opinions of authors expressed herein do not necessarily state or reflect those of the 


\title{
VI. Command Summary
}

\section{Command Summary}

\author{
MadCharacterist 16sfu12 ["machine", shot number] returns a list of \\ characteristic names for the discharge and their values. \\ Malcharacteristicshist l"machine", shot number, \\ \{characteristic , characteristic2...\}) returns a table of characteristics \\ and their values. \\ Maicharacteristics [ machine", shot number] returns a list of \\ characteristic names for the discharge. \\ Mdicharacteristics [ machine"] returns a list of all unique characteristic names \\ for the machine. \\ Mdicharacteristicvalue [ machine", shotnumber, \\ "characteristic"] returns the value of the characteristic for this discharge. \\ Maicommand [ returns the command line of the last command submitted to the shell. \\ Maicomment [comment number] returns the comment referred to by comment \\ number as a string. \\ Ma1Defindtion [ "word"] returns the definition of word as a string. \\ MaLDictionary [ pattern"] returns list of words that match the pattern and have \\ definitions in the dictionary. \\ MdiD Is chargesByReference [ reference number"] returns a list of machine \\ names and shot numbers associated with a particular reference.
}

MdiDischargesByType [ machine", "keyword"] returns a list of all shot numbers for a machine that are described by the same keyword.

Maidischargeconditionstable ["machine", shot number] returns a table of discharge characteristics with headings for a particular shot.

Mdidischargeconditions [ machine", shot number] returns the nominal Bt. Ip, NeBar, VLoop, Gas, Zeff, and number of time slices for a given discharge in the indicated order.

MdiDischargesful1 [ "machine"] returns a formatted table of shot numbers for given machine including the characterization data for each discharge.

MdiDischargesTable [ machine"] returns a table of shot numbers with characterization data including column headings.

MdiDischarges [ "machine" ] returns a list of shot numbers for given machine. 
Ma iErrormessage [ - If the last query generated an error message from the database engine or from the RPC communication layer, this command can be used to retrieve the full text of the message.

MdiHeadings [ ] returns the DBMS table headings from the last command.

Malkeywords ["machine", shot number] teturns a list of key words describing the discharge.

Mdikeywords ( machine"] returns a list of all unique key words for the machine.

MailastQuery [] returns the exact form of the last query submitted.

MdiMachineparameters ["machine", shot number] returns a list of the names of all machine parameter sets and machine parameters and their values for this shot.

MalMachineparameters ["machine"] returns a list of the names of all the names of themachine parameter sets for the machine.

Maimachines [] returns a list of machines in the database.

Mdimachinevaluel "machine", shot number, "machine parameter set", "machine parameter"] returns the value of a particular machine parameter.

MdiNeIF ["machine", shot number, slice:1] returns the interpolating function for generic electron density profile.

Mdineplot "machine", shot number, slice:1] returns the generic electron density profile.

MdifauxIF["machine", shot number, slice:1] returns the irterpolating function for generic auxiliary power profile.

Mdipauxplot ["machine", shot number, slice:1] returns tha generic auxiliary power profile.

Mdiperturbationstable ["machine", shot number] returns a formatted table with headings of the perturbation information.

Mdiperturbations [ machine", shot number] returns a table of perturbation information.

MaipohIF ["machine", shot number, slice:1] returns the interpolating function for generic ohmic power profile.

Maipohplot ["machine", shot number, slice:1] returns the generic ohmic power profile.

Mdiprofiledata [machine", shot number, "profile", slice:1] returns information about the profile. 
MdiproflleFit ["machine", shot number, "profile", slice:1] returns the fit parameters.

MaiprofileIf ["machine", shot number, "profile", slice:1] returns an interpolation function for a profile.

Malproflleplot ["machine", shot number, "profile", slice:1] returns a graphic object including error bars if available.

MalProfilesizel machine", shot number, "profile", slice:11 returns the number of points in a profile.

Malprofiles ["machine", shot number] returns a list of profile names for this discharge.

MdiprofileTable [machine", (shot number ist], [profile ist] ] returns a table of profile names for each shot on the list in which each has the same radial points.

Mdiprofilexuabel ["machine", shot number, "profile"] retums the name of independent variable for the profile.

MdiprofileXY["machine", shot number, "profile", slice:1] returns the a list of $x-y$ pairs.

Maiprofile ["machine", shot number, "profile", slice:1] returns a list of $x, d x, y, d y$ four-tuples.

Maiesprint [ -graphic- ] sends postscript graphics to a particular Apple Laser Writer printer.

Mdiquery I "Query..."] submits the query given as a text string to the database and returns the resulting table in Mathematica list form.

Md IRawReturn[] returns the uiprocessed result from the MDIClient program.

Mdikeferences [ machine"] retums a list of reference numbers and the references associated with a particular machine.

MdiRows [] returns the number of rows in the last query result table.

Mdisource ["machine", shot number, "profile"] returns information about the person or group responsible for this measurement.

Mdisummary ["machine", shot number] returns a graphic array of plots for $\mathrm{Te}, \mathrm{Ti}$, ne, Poh, Paux and a table of the nominal discharge parameters.

MaiTeIF ["machine", shot number, slice:1] returns the interpolating function for generic electron temperature profile.

MdiTeplot ["machine", shot number, slice:1] returns the generic electron temperature profile. 
MdiTiIF [ "machine", shot number, slice:1] returns the interpolating function for generic ion temperature profile.

MdiTiplot [ machine", shot number, slice:1] returns the generic ion temperature profile.

MdiToSpreadsheet [ "fi lename", "data"] writes data table to an external file in a form suitable for a Macintosh spreadsheet program.

\section{References}

1 S. Wolfram, "Mathematica: a system for doing mathematics", Addison-Wesley, Redwood City, 1991.

2 W.H. Miner, Jr., J.C. Wiley, D.W. Ross, The University of Texas at Austin, Report FRCR 323, 1988.

${ }^{3}$ SQL Language Reference Manual, Oracle Corp., 1990.

4 John R. Corbin, "The Art of Distributed Applications", Springer-Verlag, New York, 1990

5 SQL*Plus User's Guide and Reference, Oracle Corp., 1989.

6 Unix man pages.

7 Jack J. Dongarra, Eric Grosse, "Distribution of Mathematical Software Via Electronic Mail", netlib, 1985.

8 W.H. Miner, Jr., R.T. McCann, and J.C. Wiley, Bull. Am. Phys. Soc. 36, 2436 (1991).

${ }^{9}$ Douglas Comer, "Internetworking with TCP/IP", Prentice-Hall, Englewood Cliffs, 1988. 
Appendix I - MFEDB diagram.

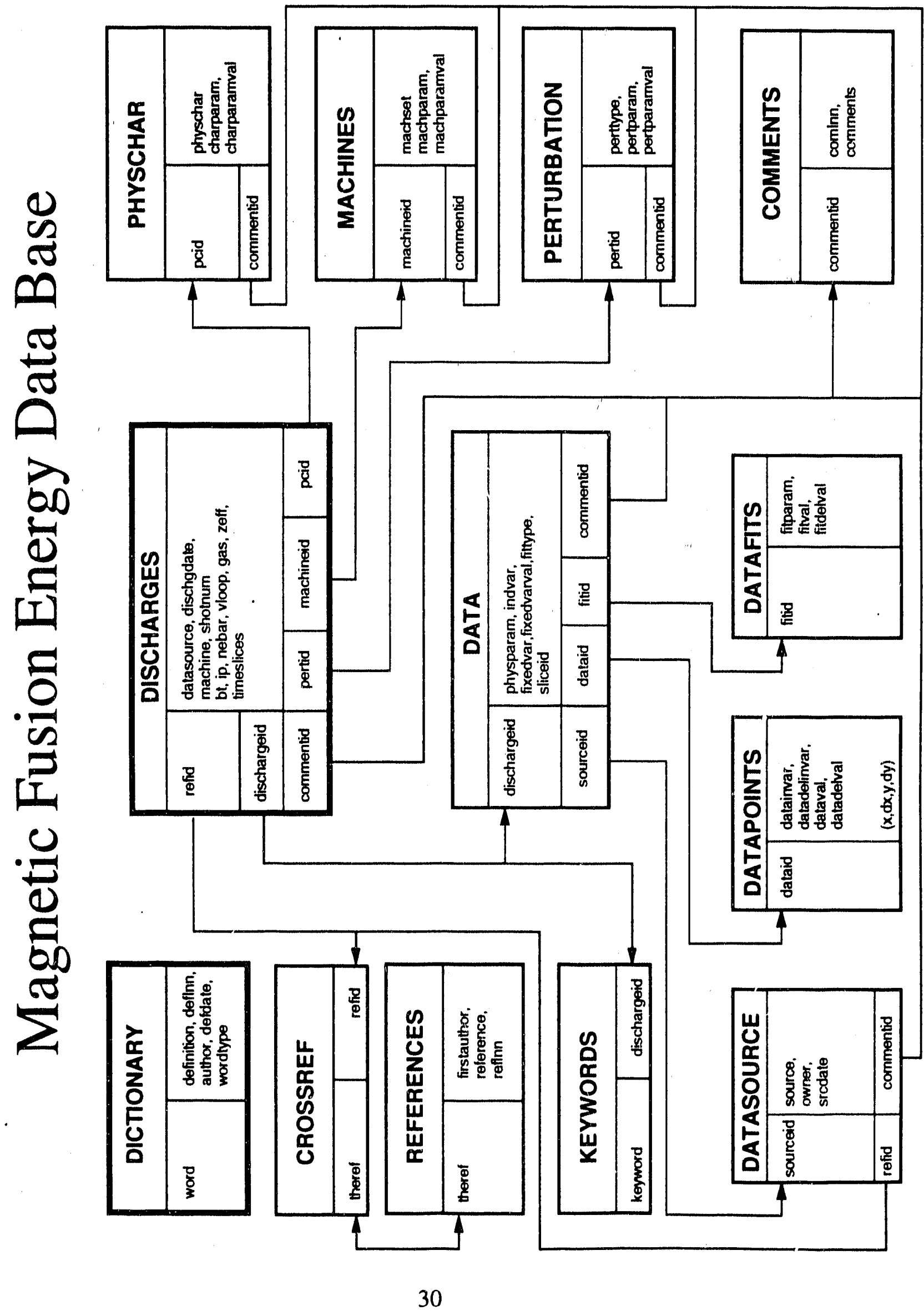




\section{Appendix II - The MdiClient code.}

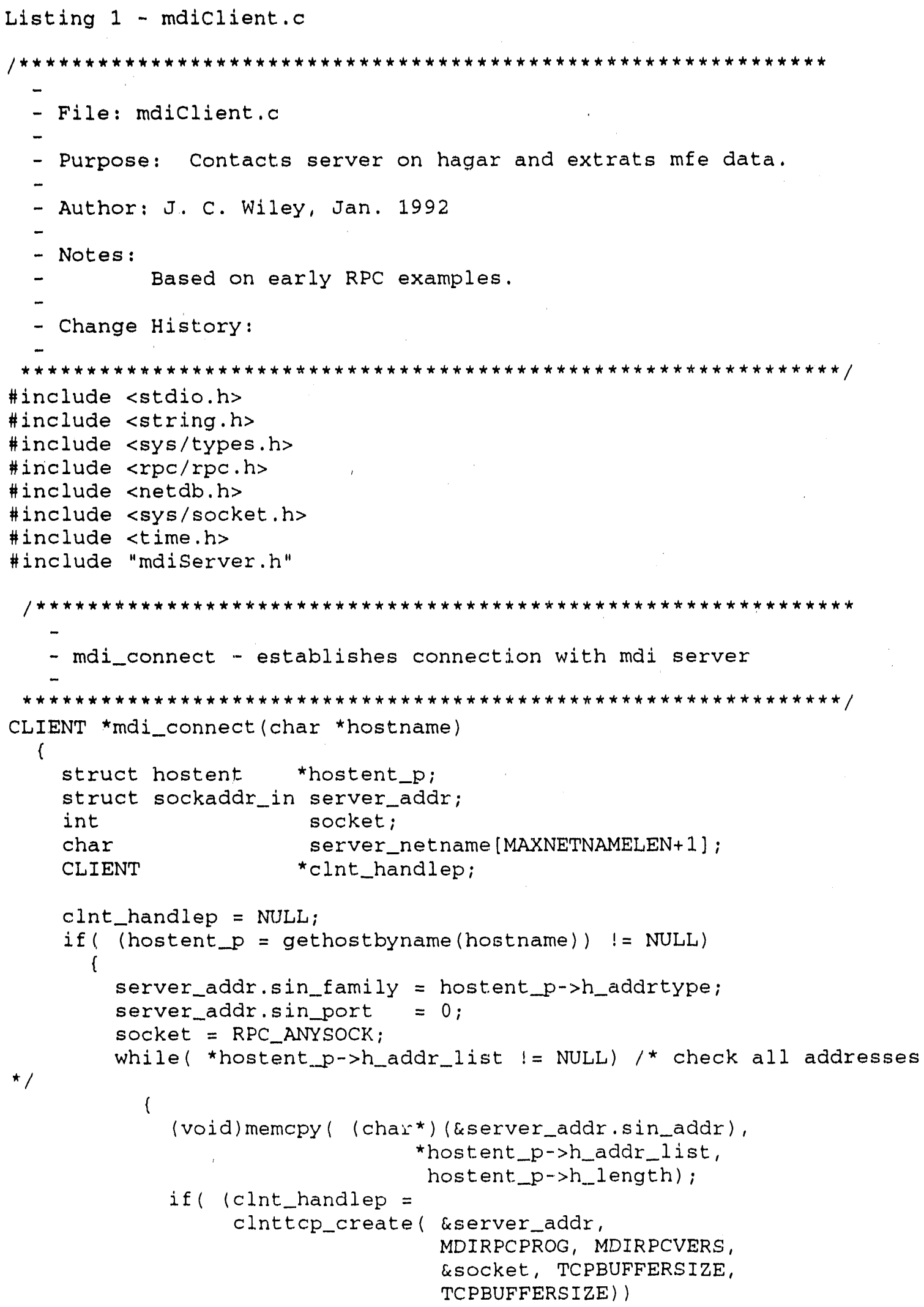




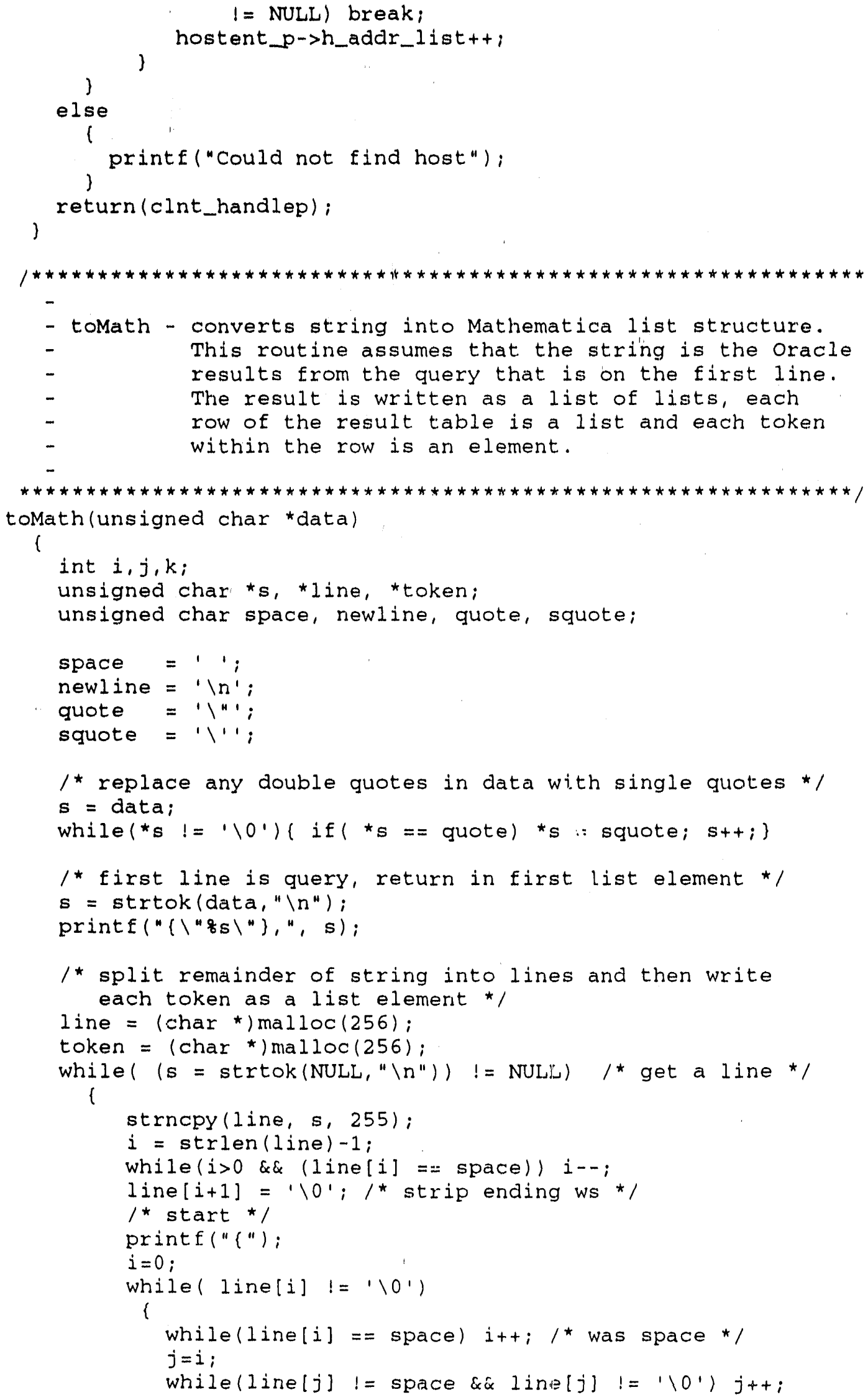




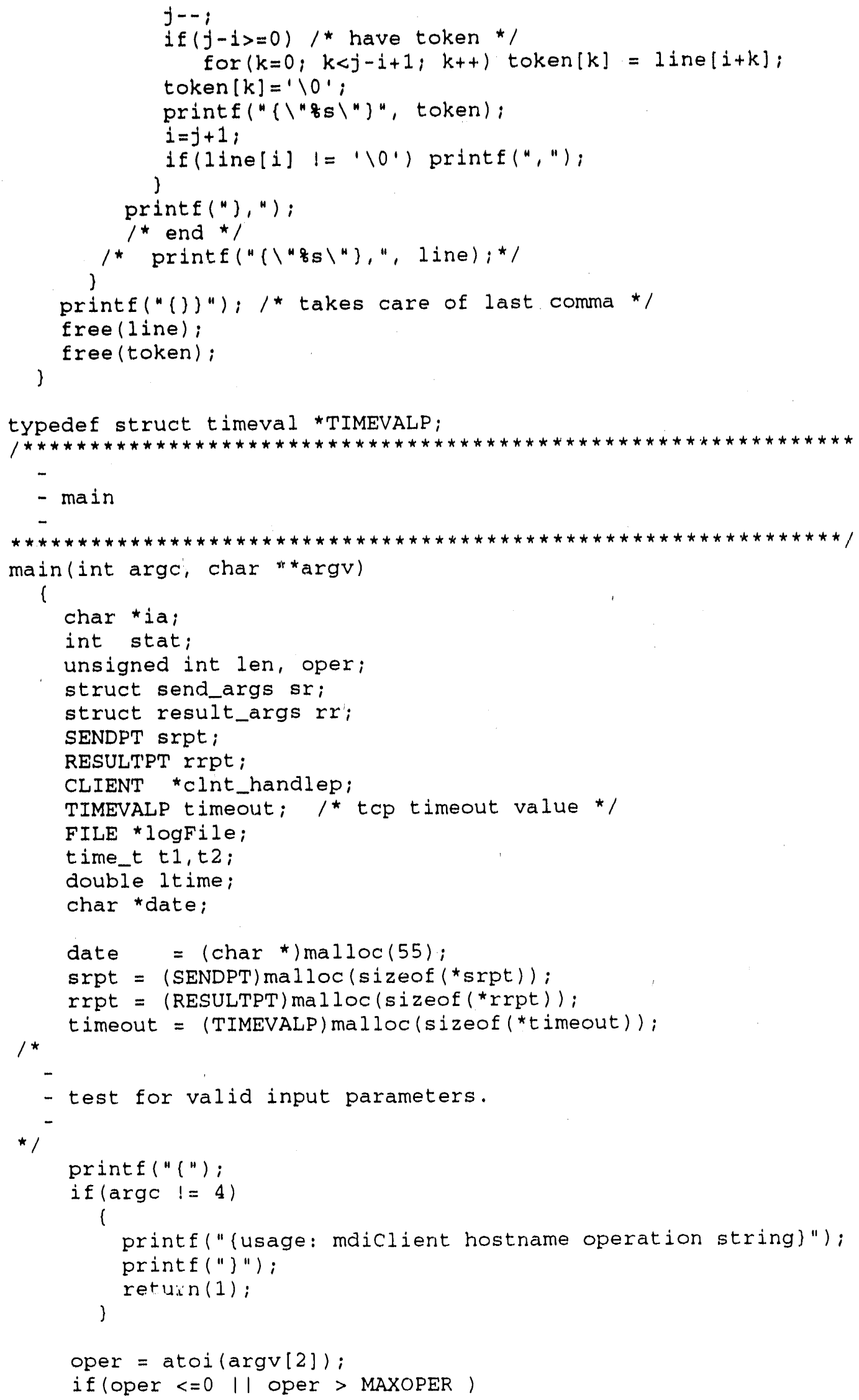




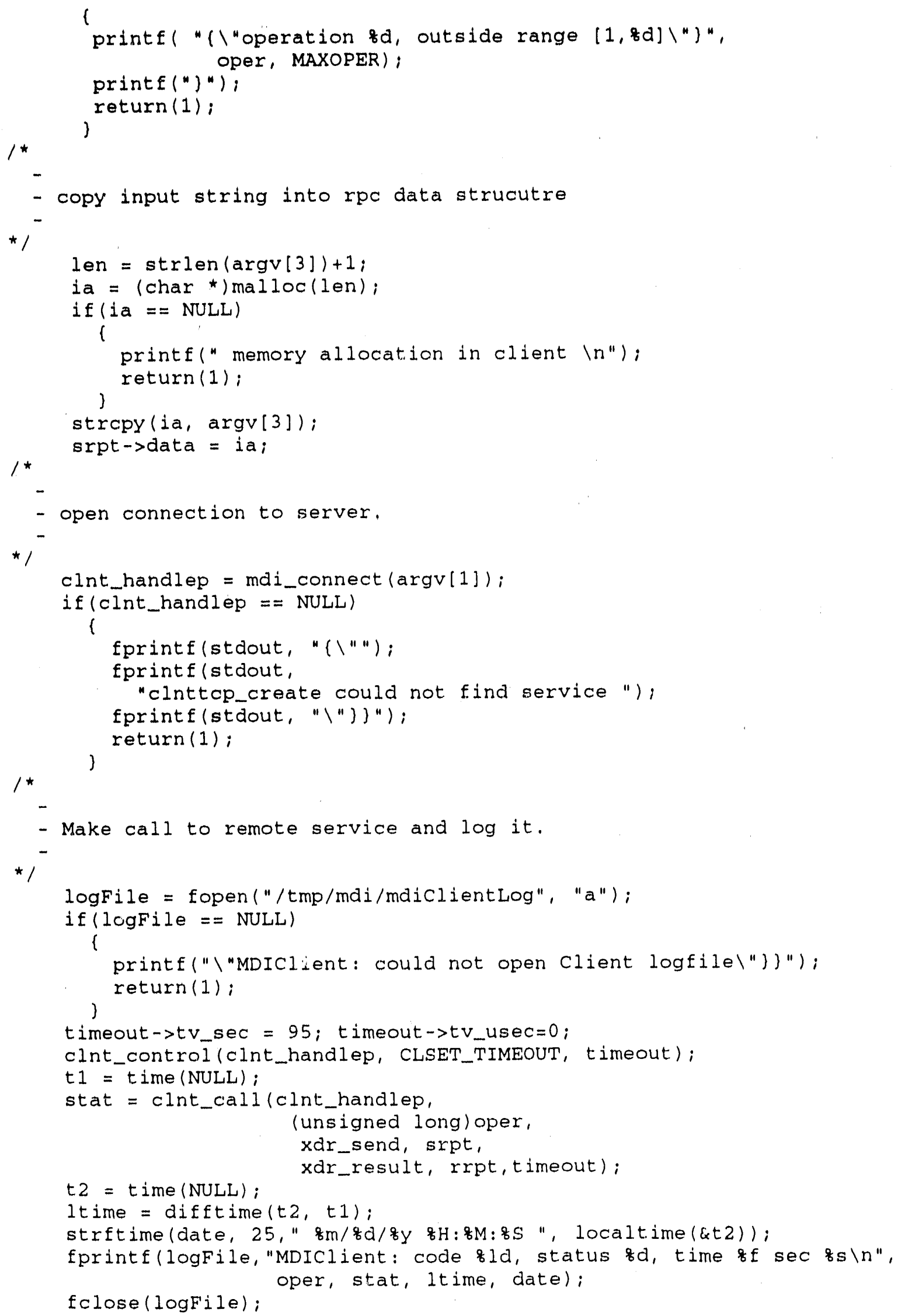




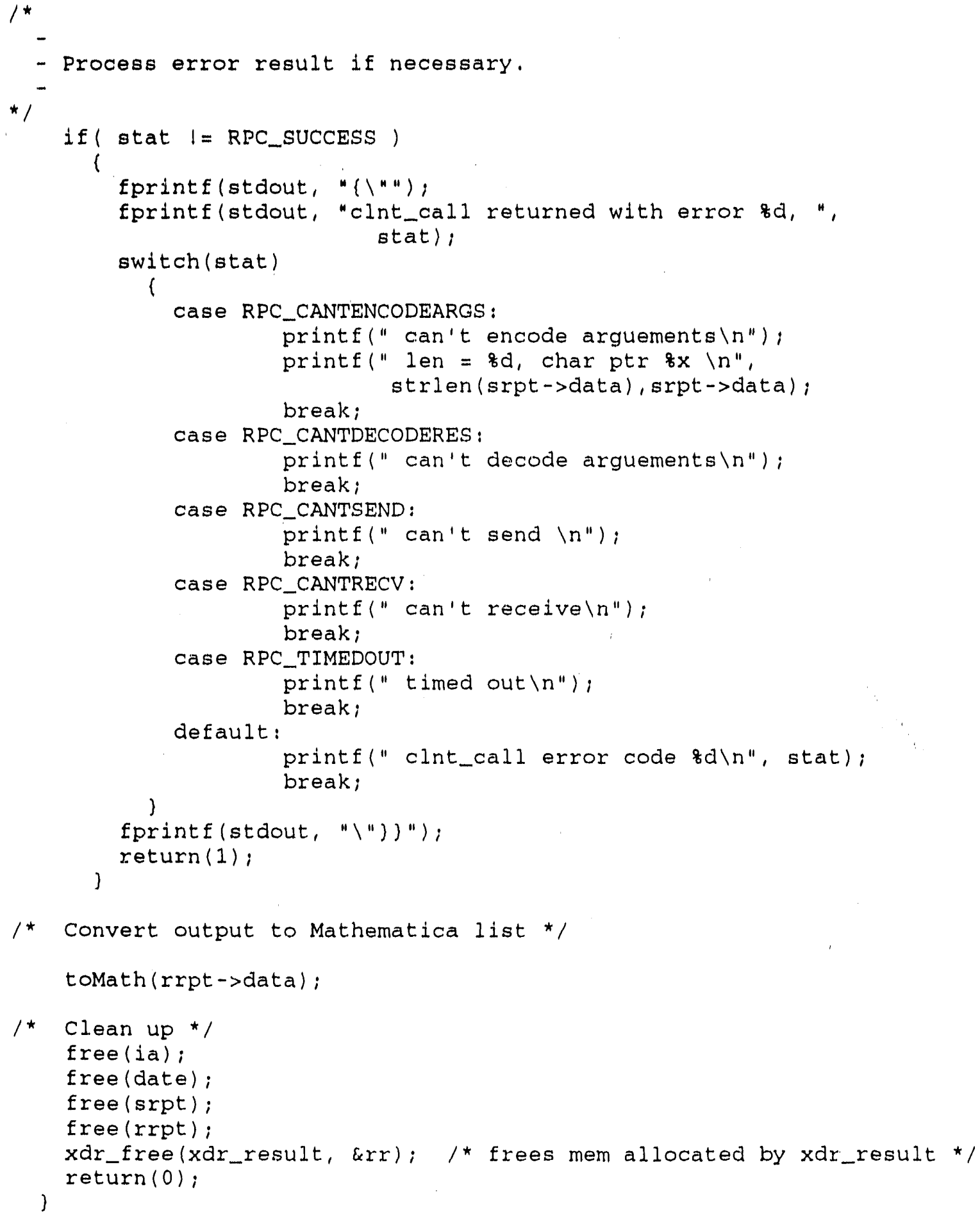




\section{Appendix III - Mdi server listings}

\section{Listing 1 - mdiServer.h}

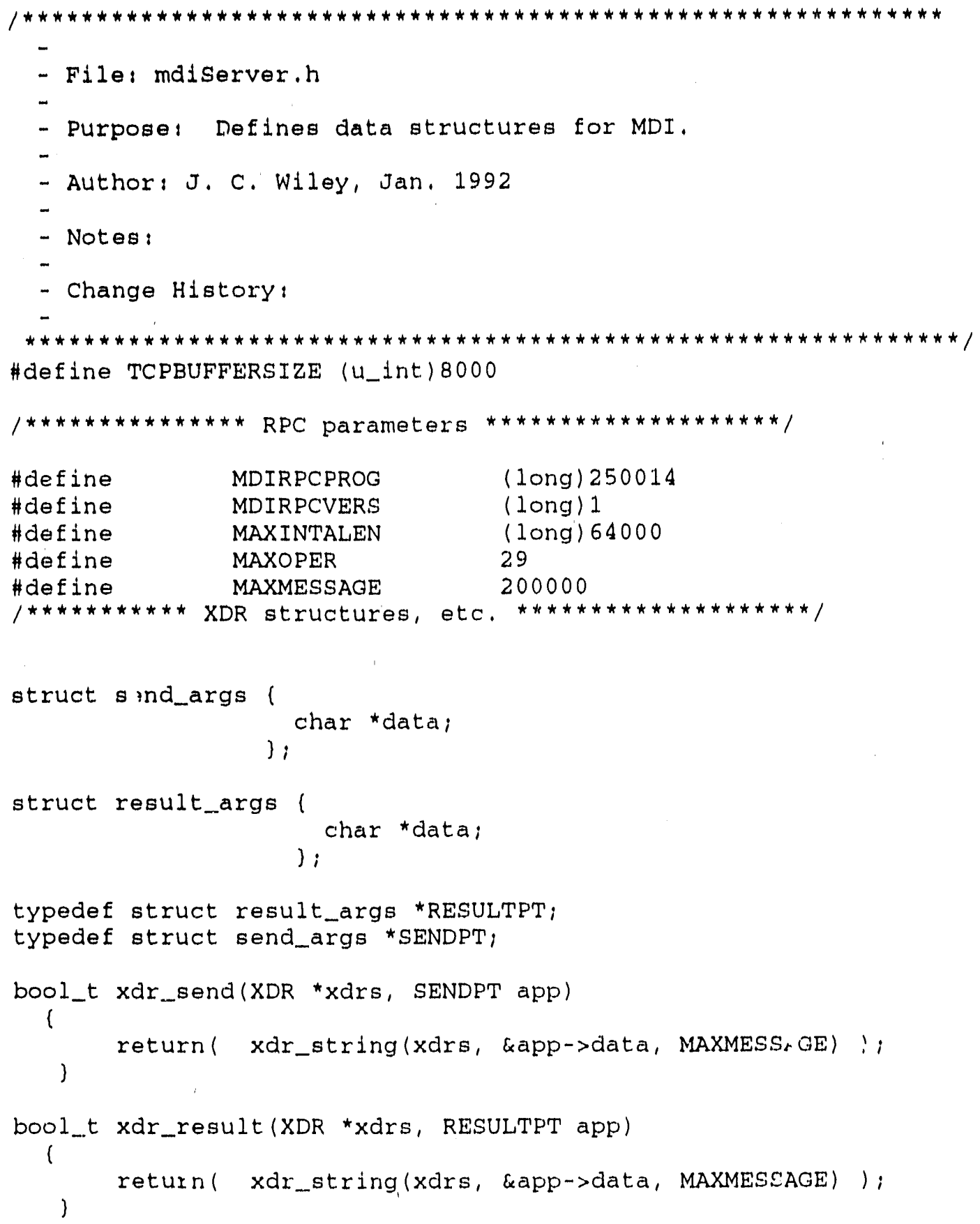




\section{Listing 2 - mdiServer.c}

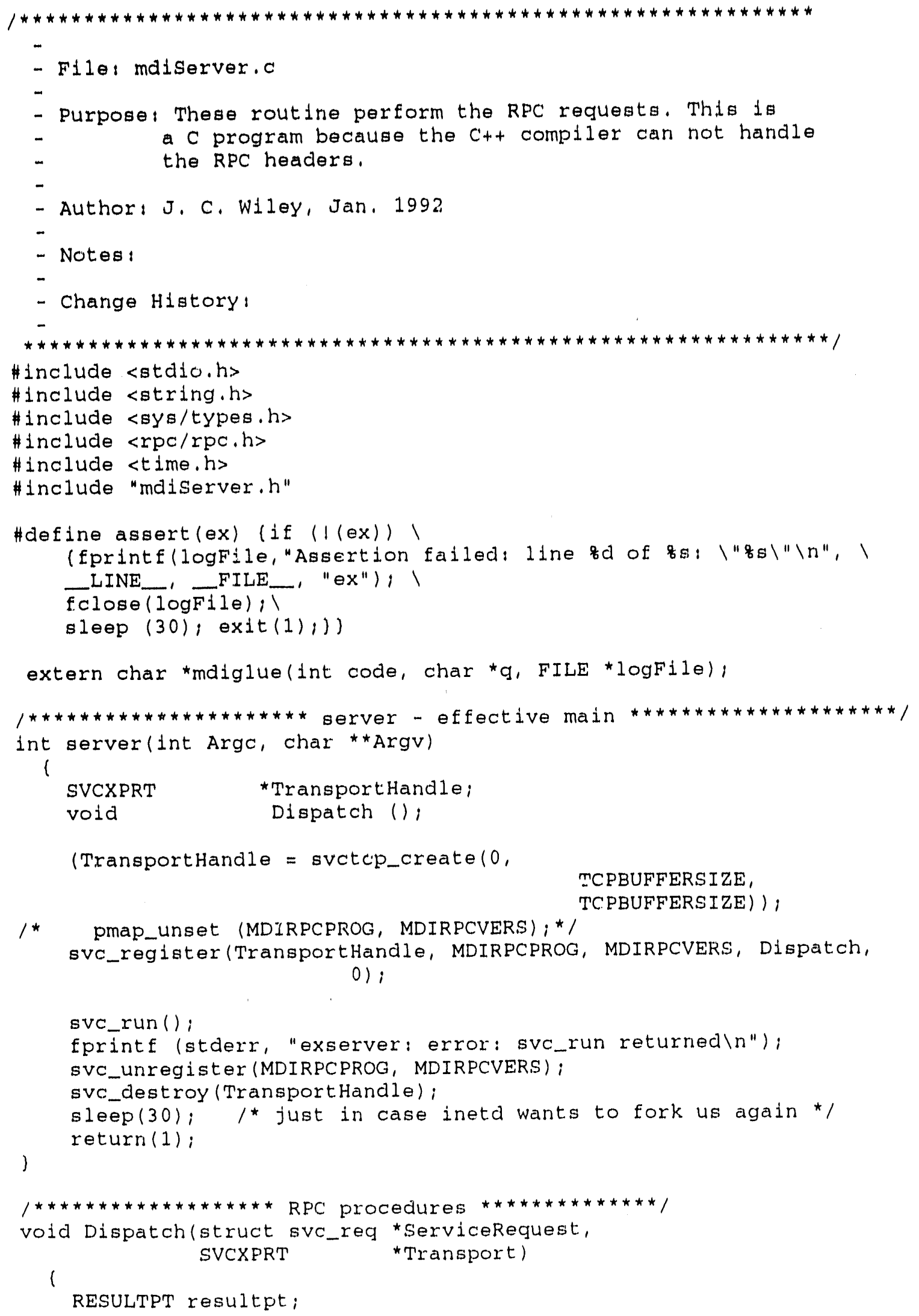




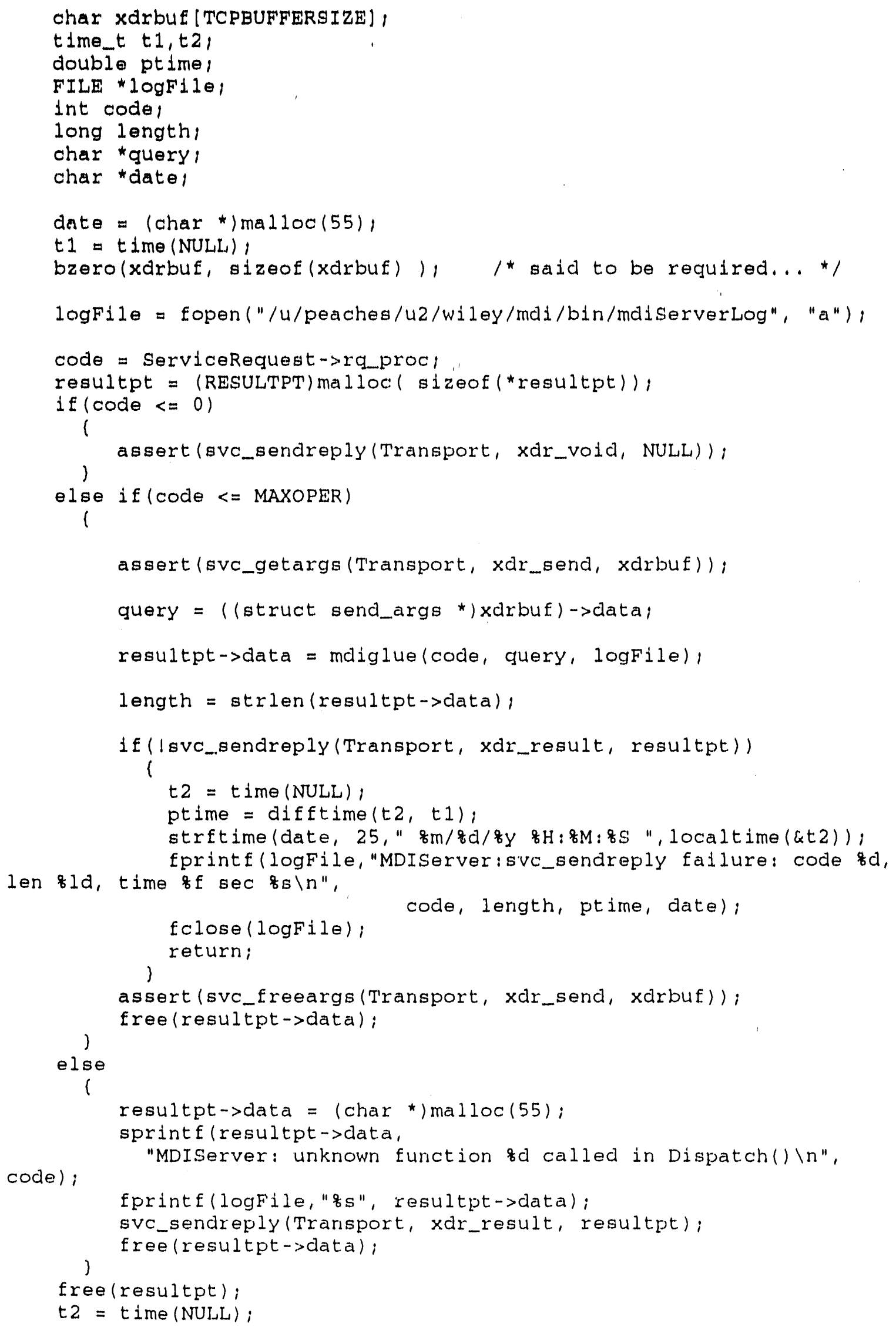


ptime $=\operatorname{difftime}(t 2, t 1)$

stretime (date, 25," $8 \mathrm{~m} / 8 \mathrm{~d} / 8 \mathrm{y}$ \% 8 : $8 \mathrm{M}: 8 \mathrm{~s}$ ", localtime(\&t2)),

fprinte(logfile, "MDIServer: code $8 d$, len $81 d$, time of sec $8 s \backslash n$ ",

Eclose $(\log F 11 \theta)$;

code, length, ptime, date);

freo (date),

)

return 


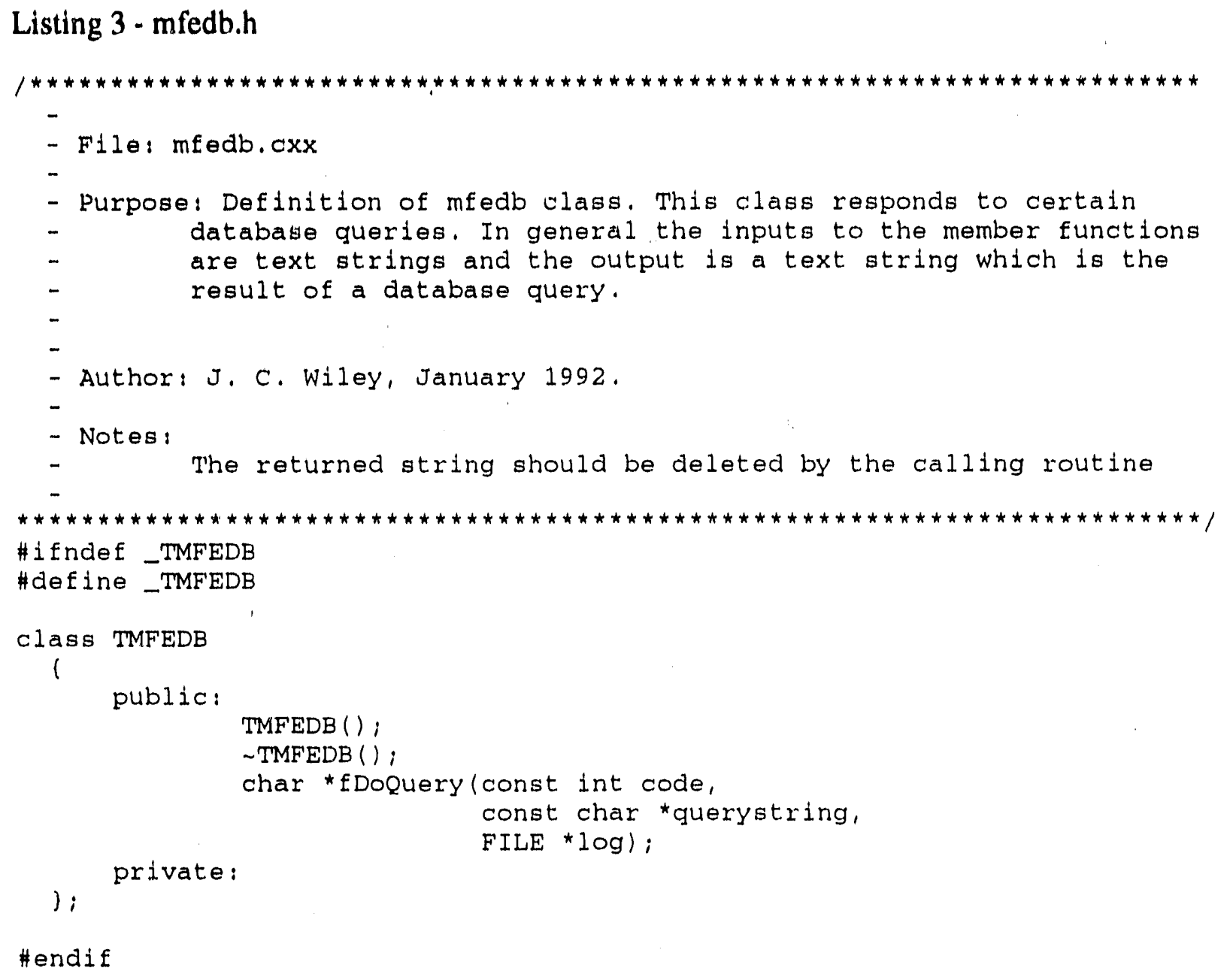




\section{Listing 4 - mfedb.cxx}

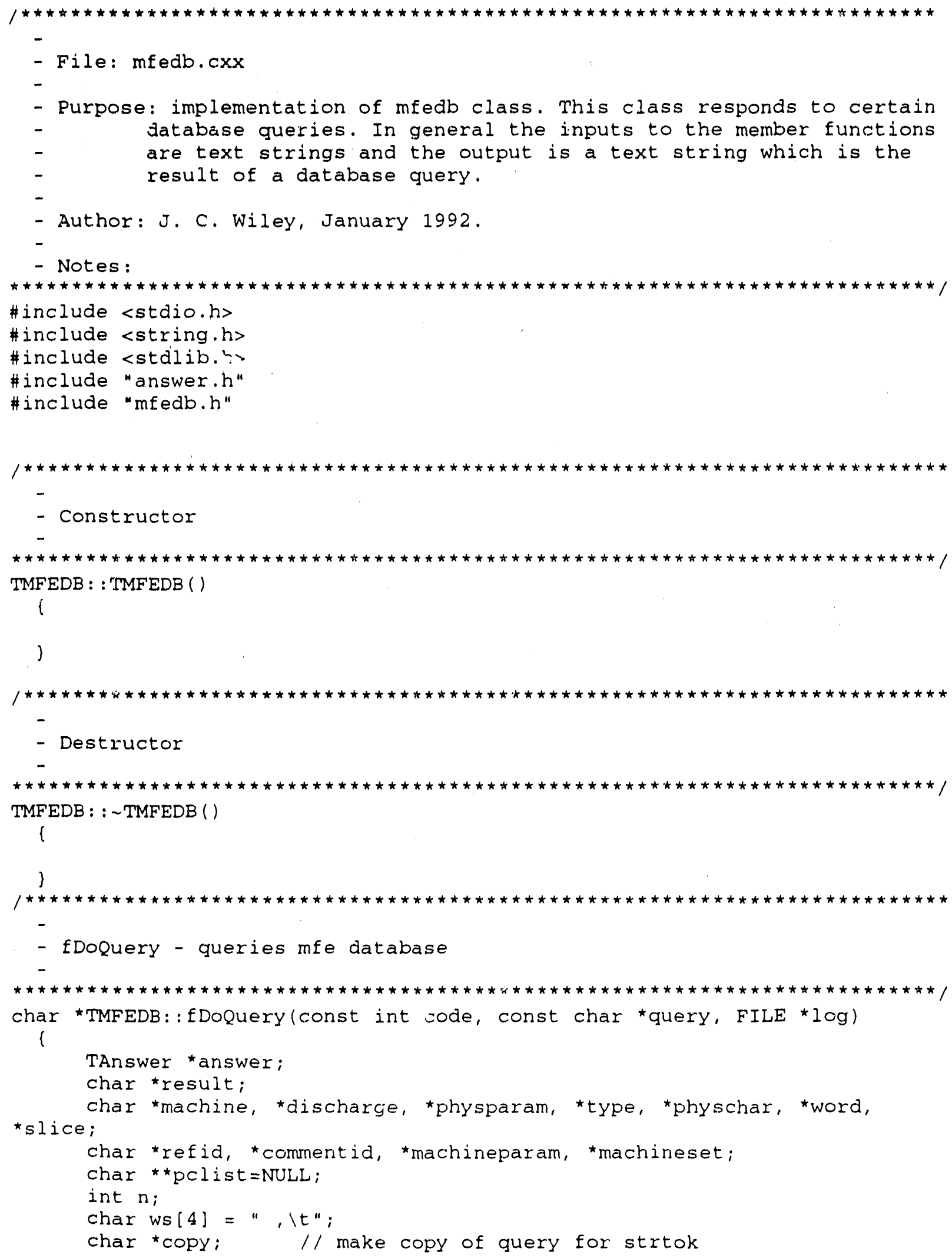




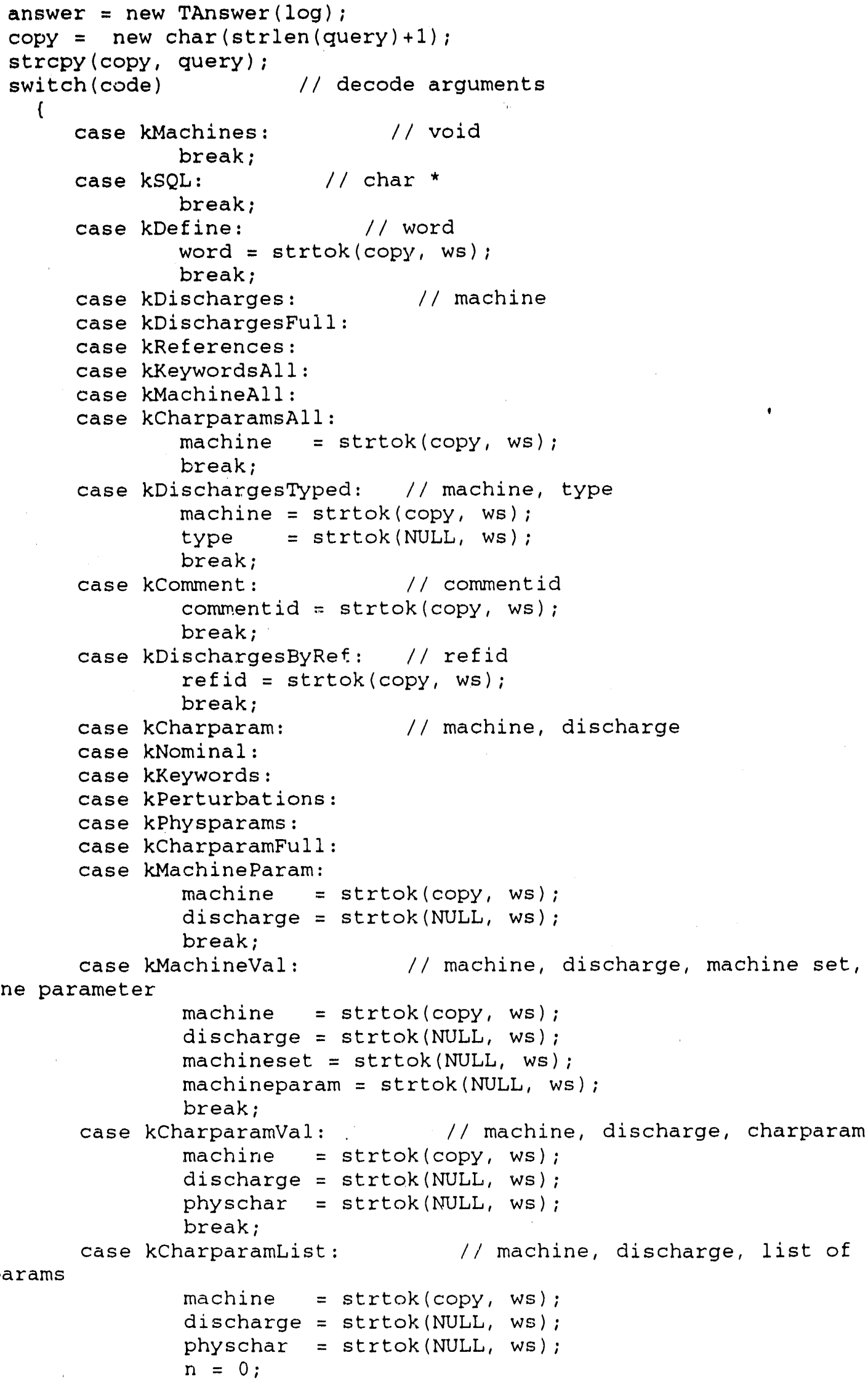




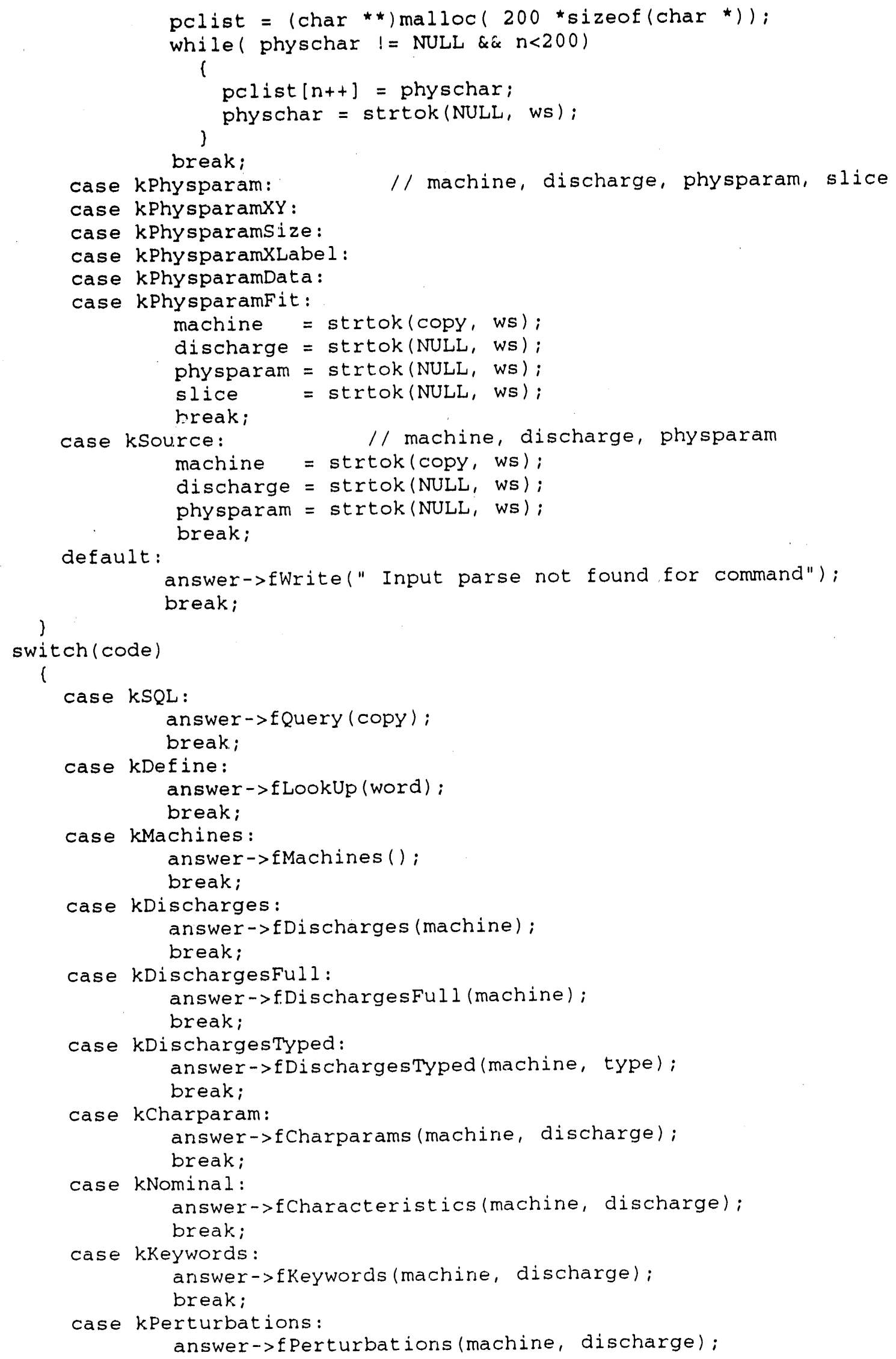




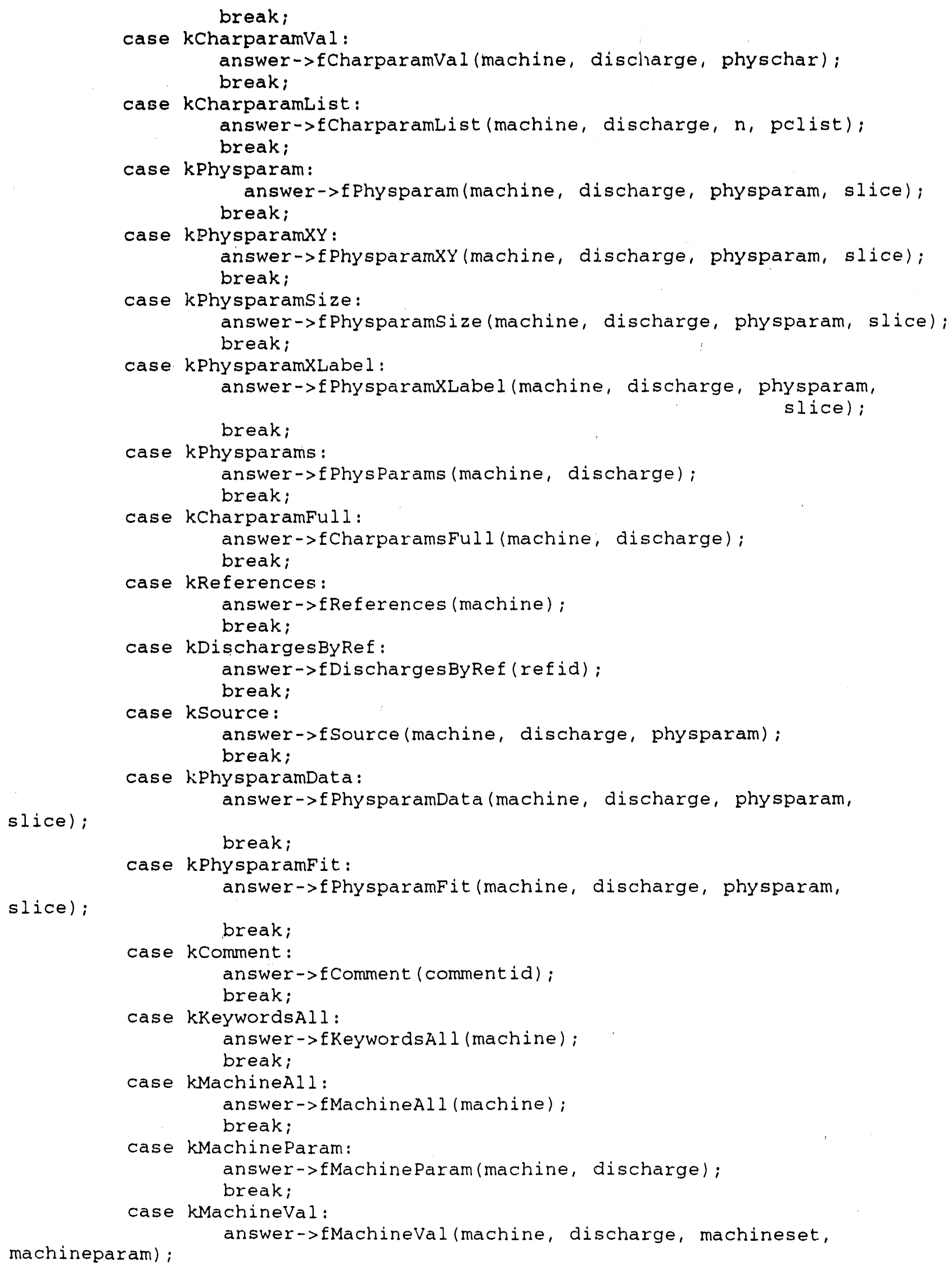




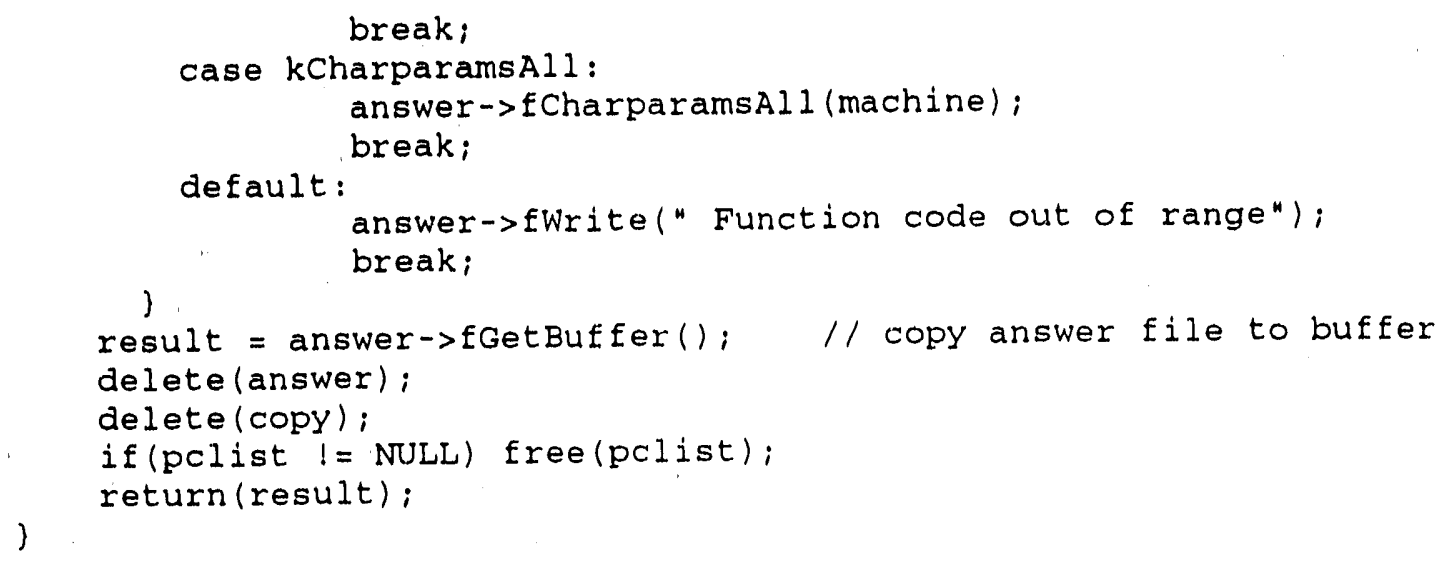




\section{Listing 5 - answer.h}

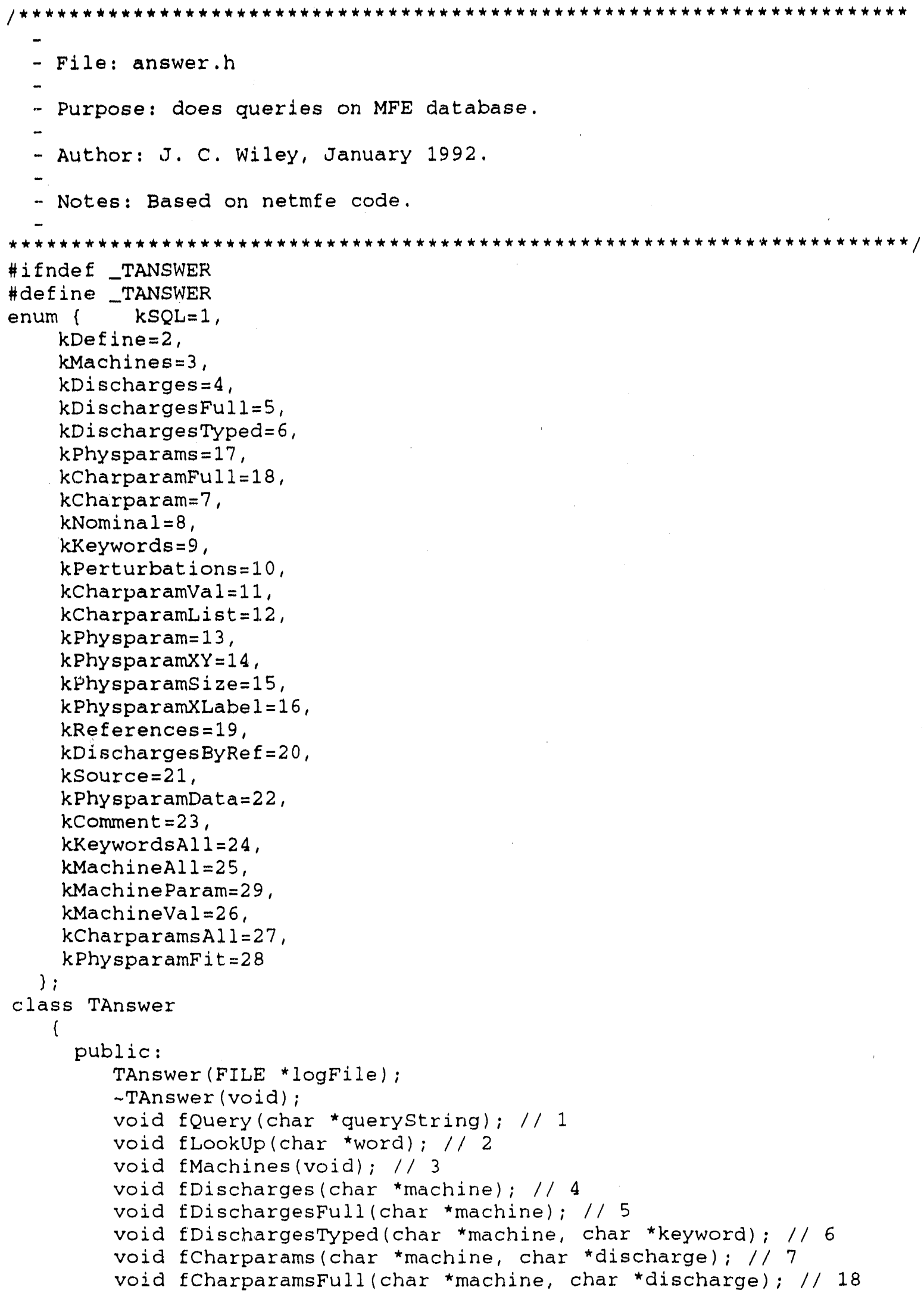




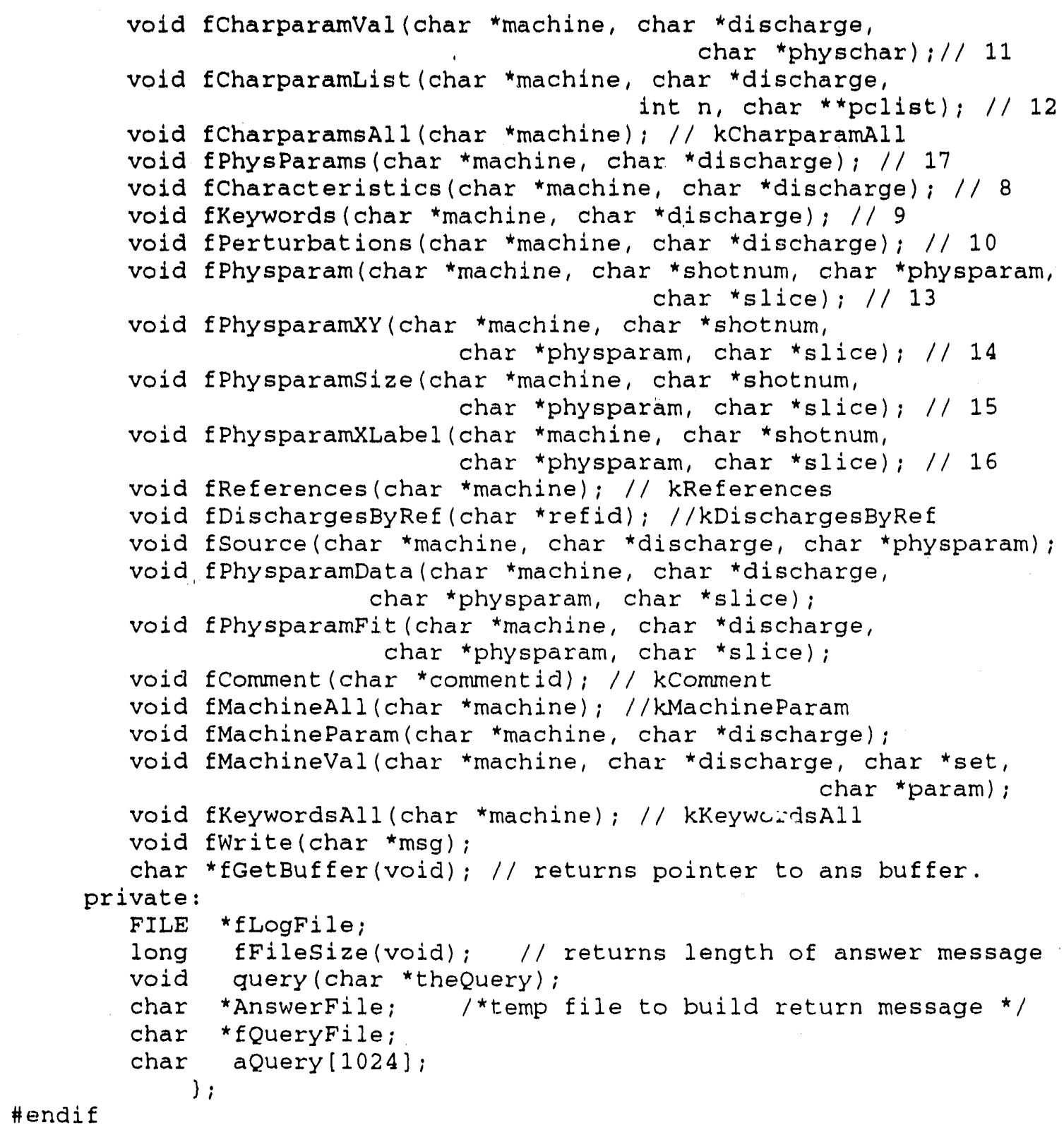




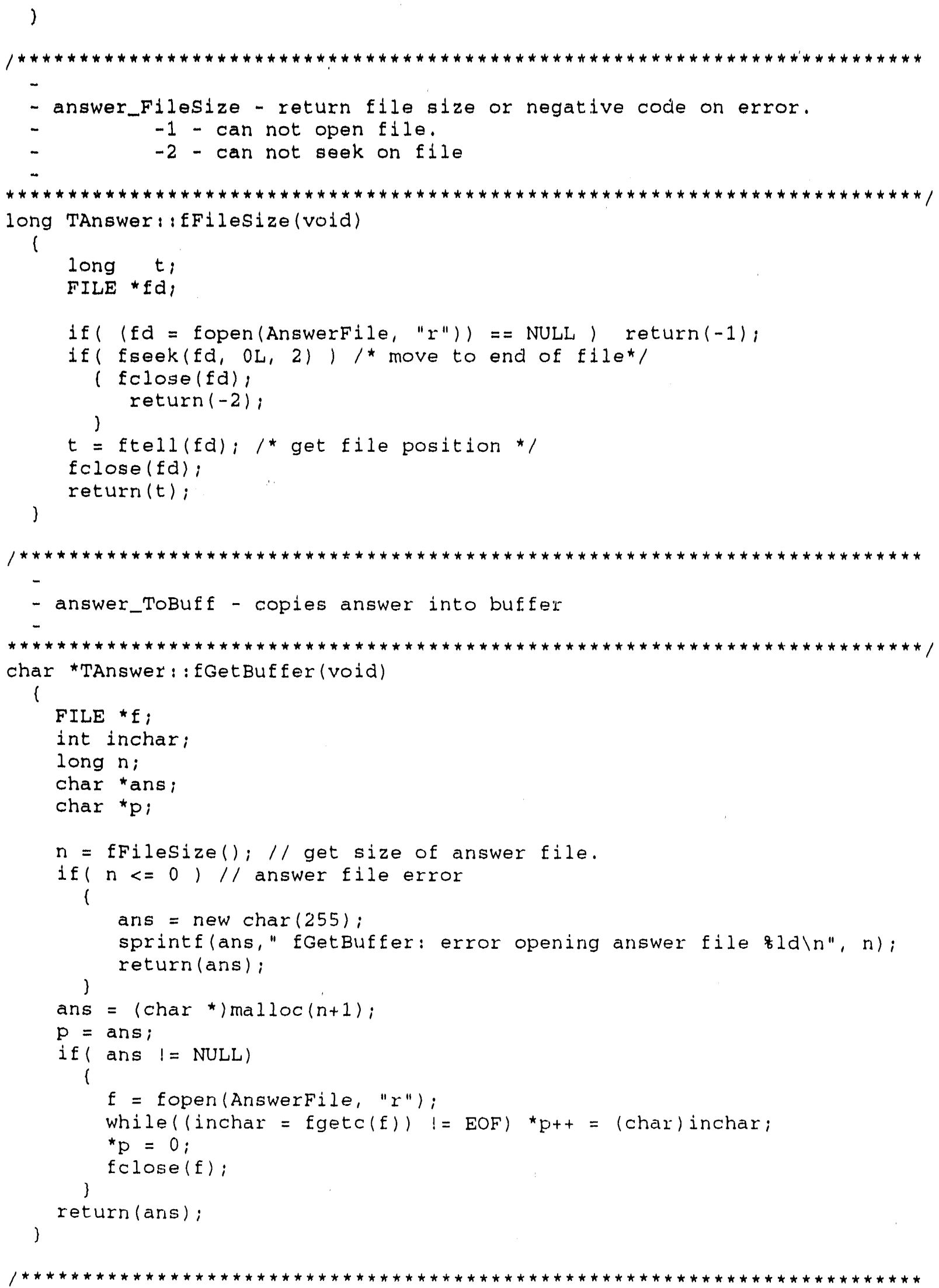


- fWrite - writes string to answer file

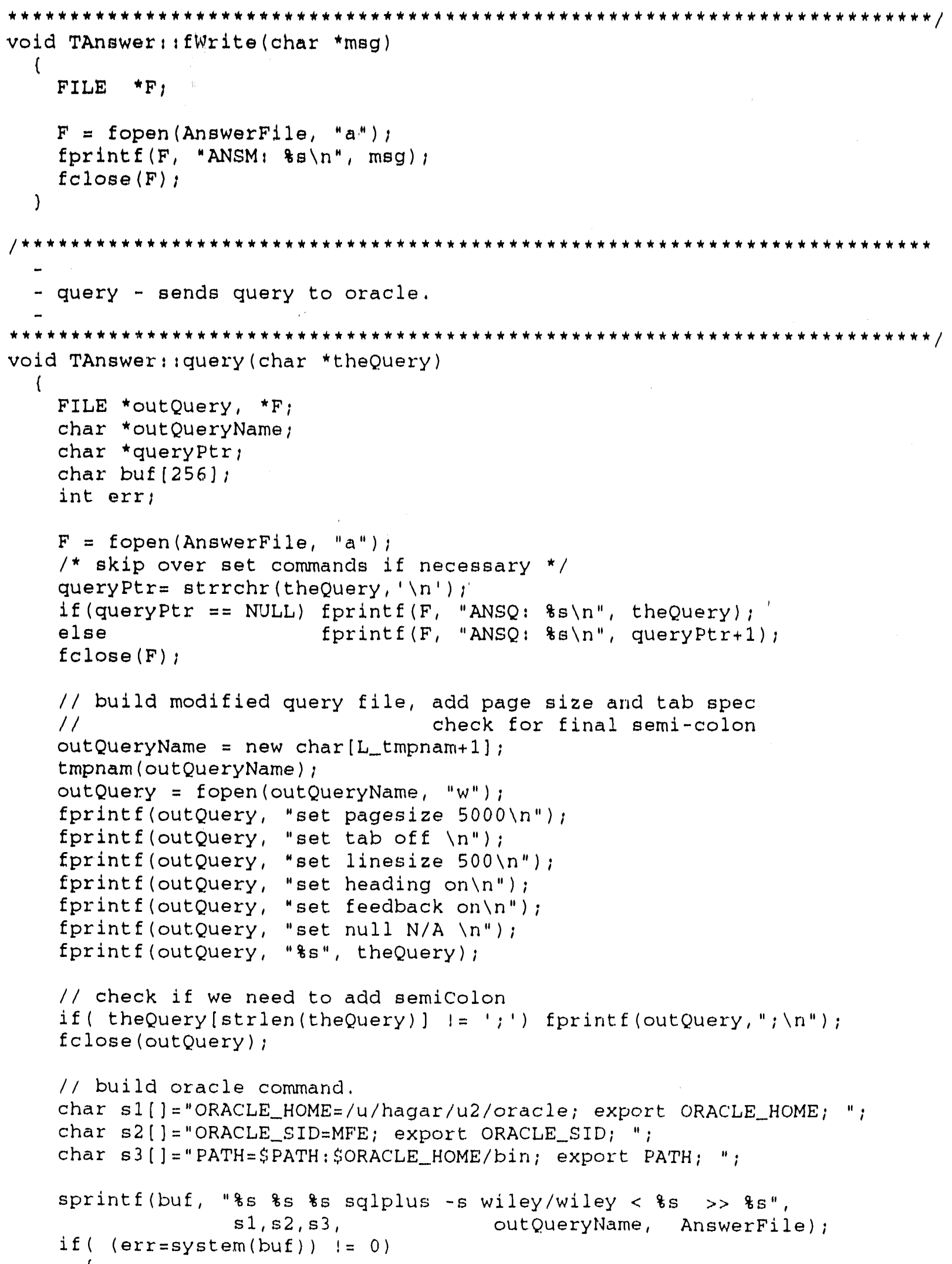




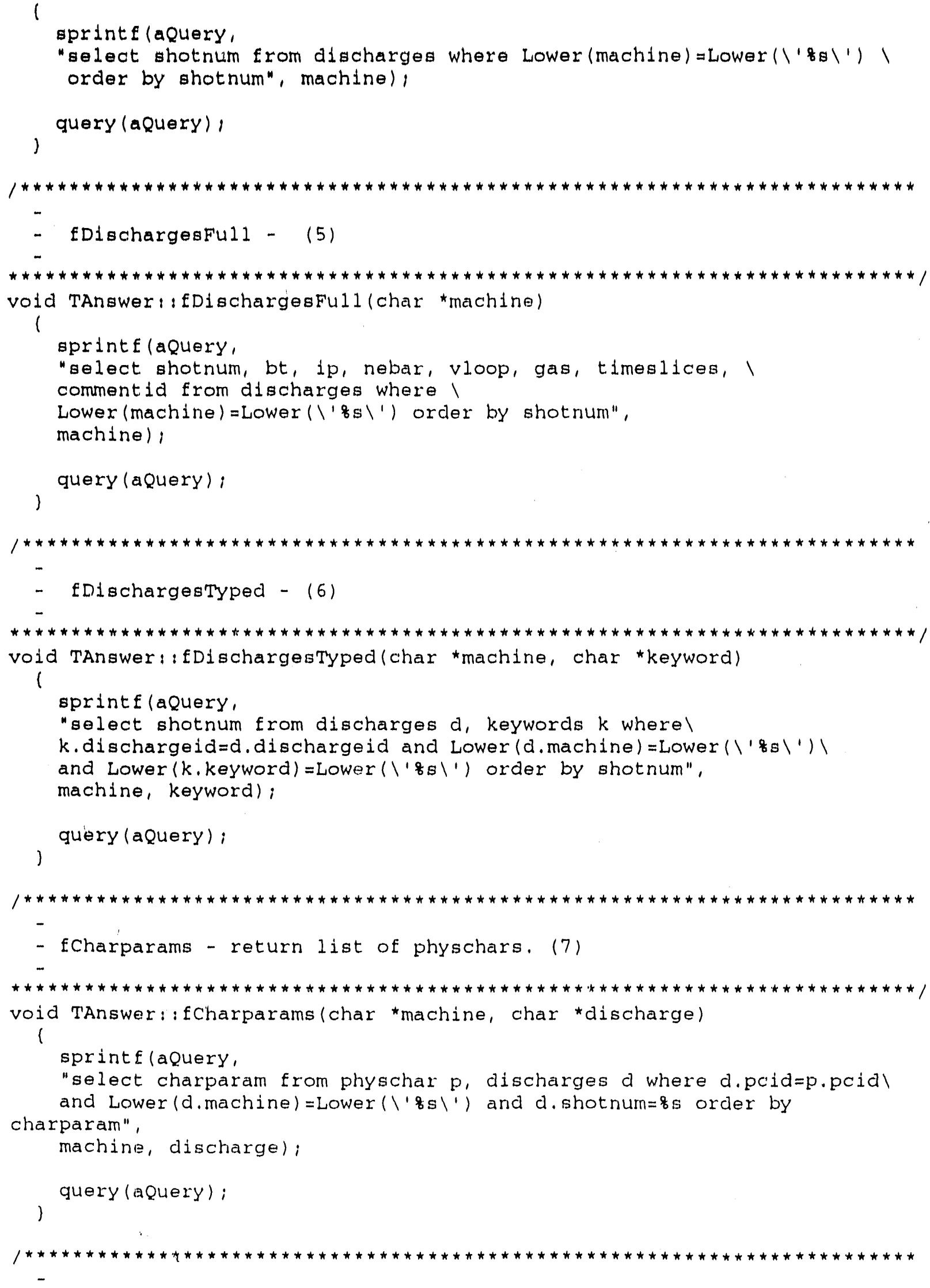




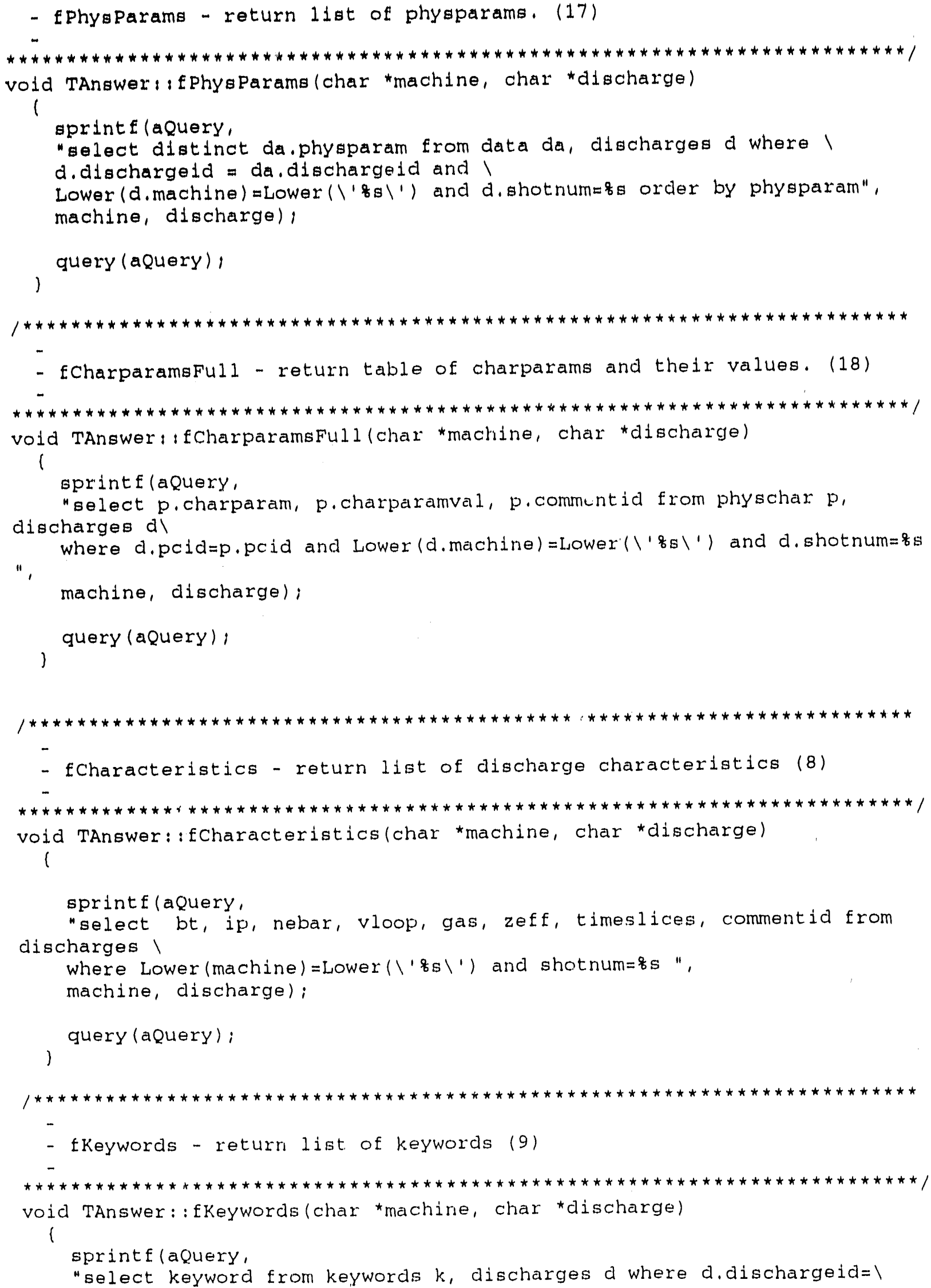




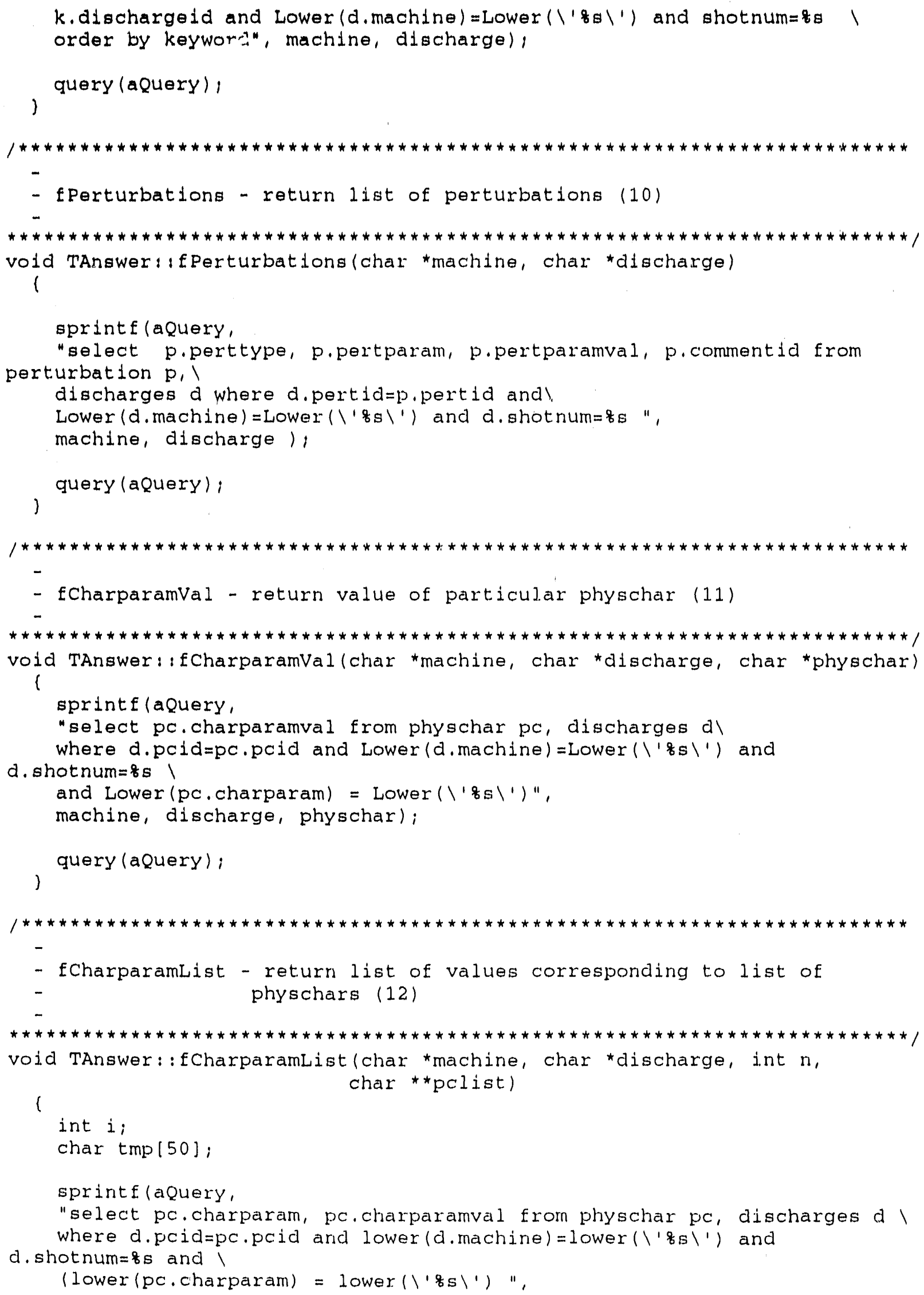




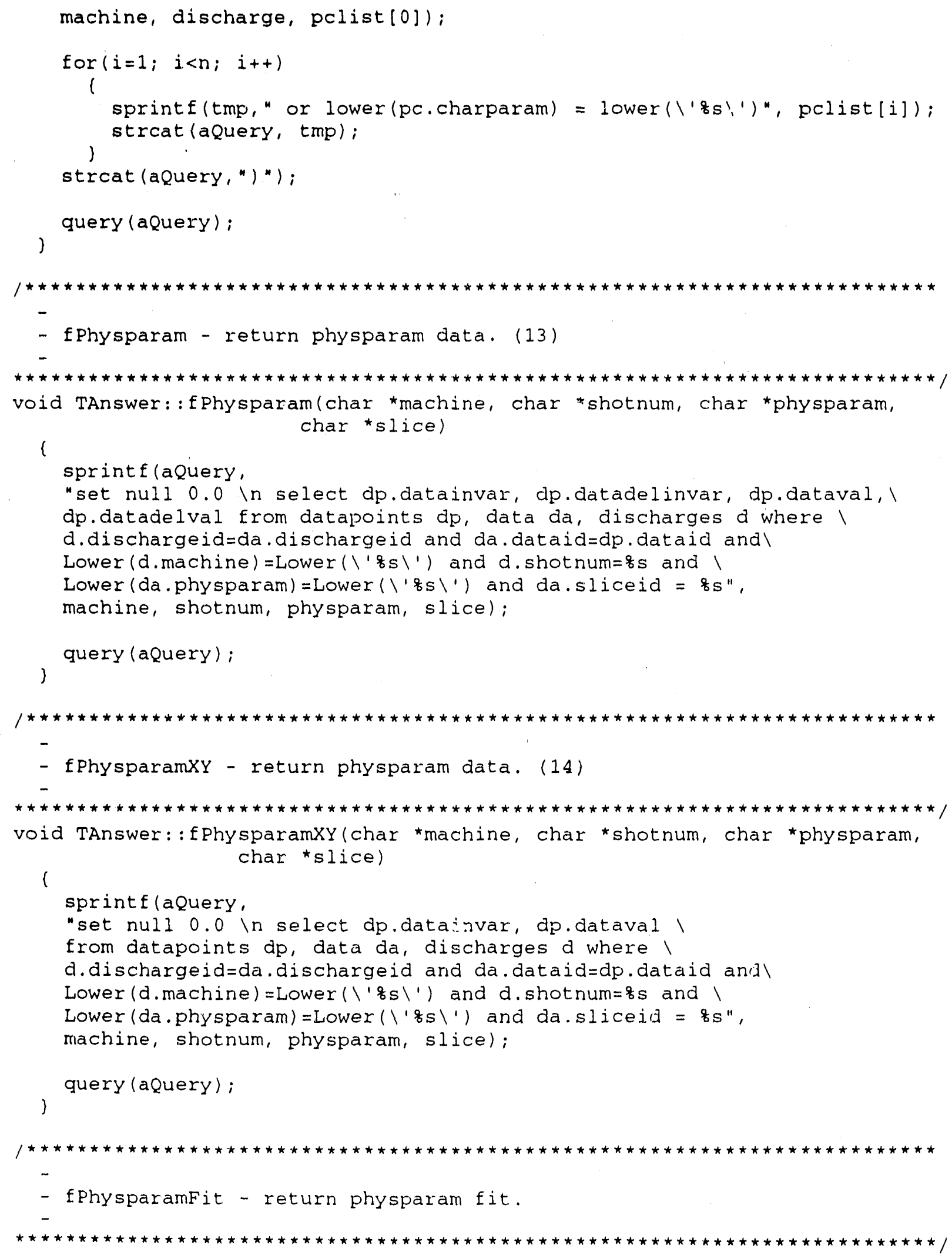




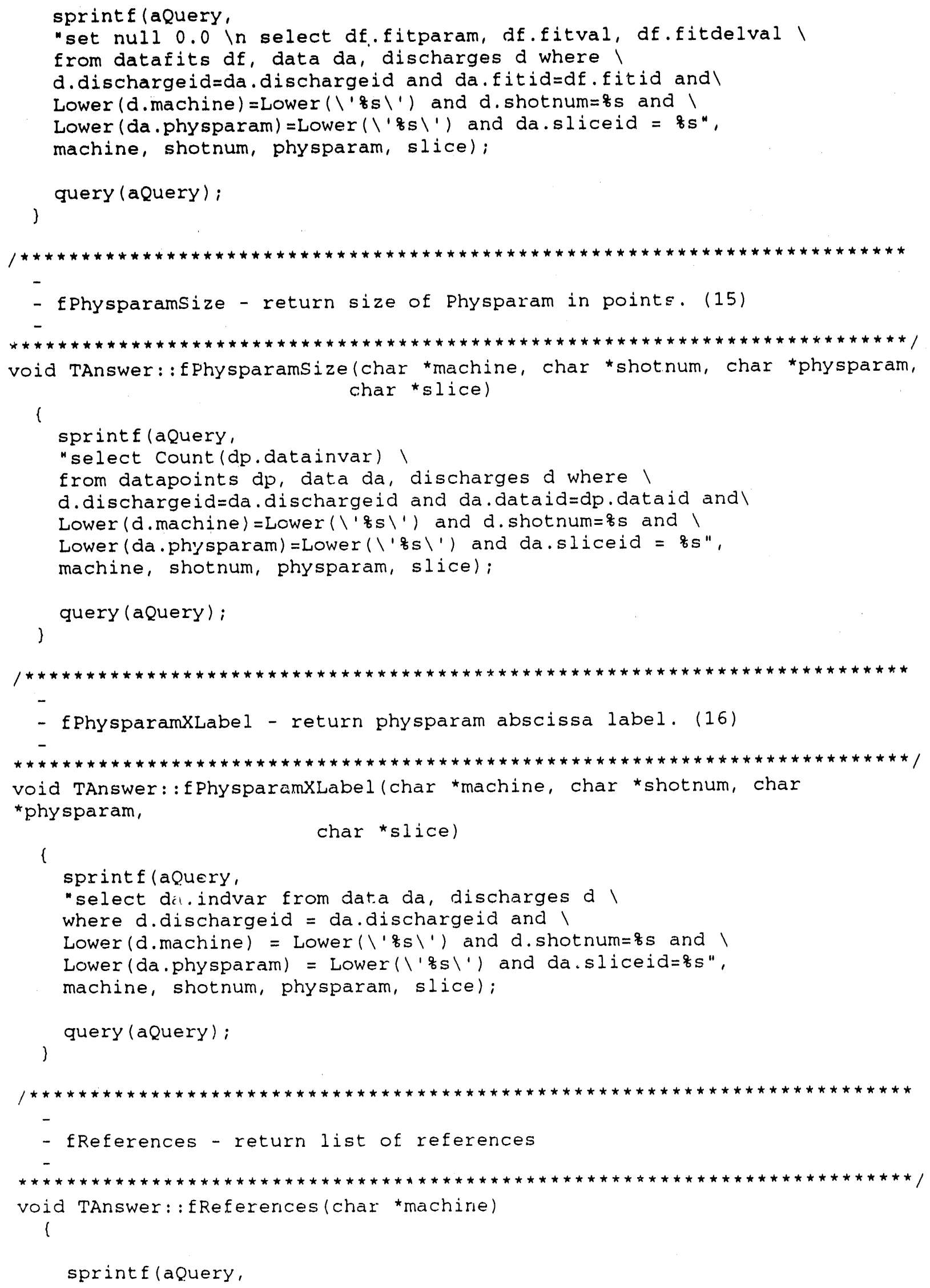


"select unique $r$.theref, r.firstauthor, r.reference, r.reflnn । from discharges $d$, crossref $c$, references $r$ where c.refid = d.refid and 1

c.theref $=r$.theref and lower $(\backslash, 8 s \backslash 1)=$ lower (d.machine) $\mid$

order by r.theref, r.firstauthor, r.reflnn", machine);

)

query (aQuery);

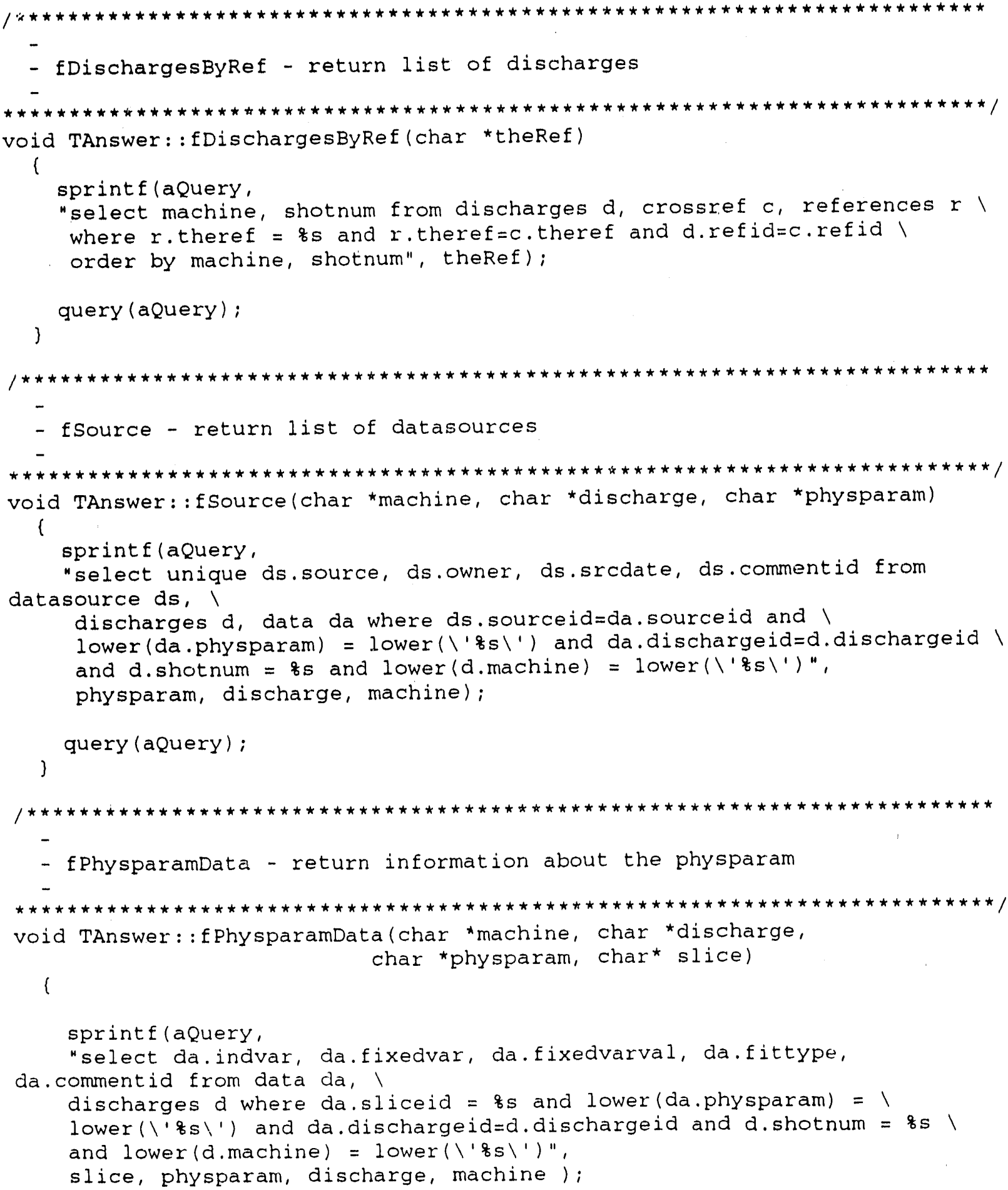




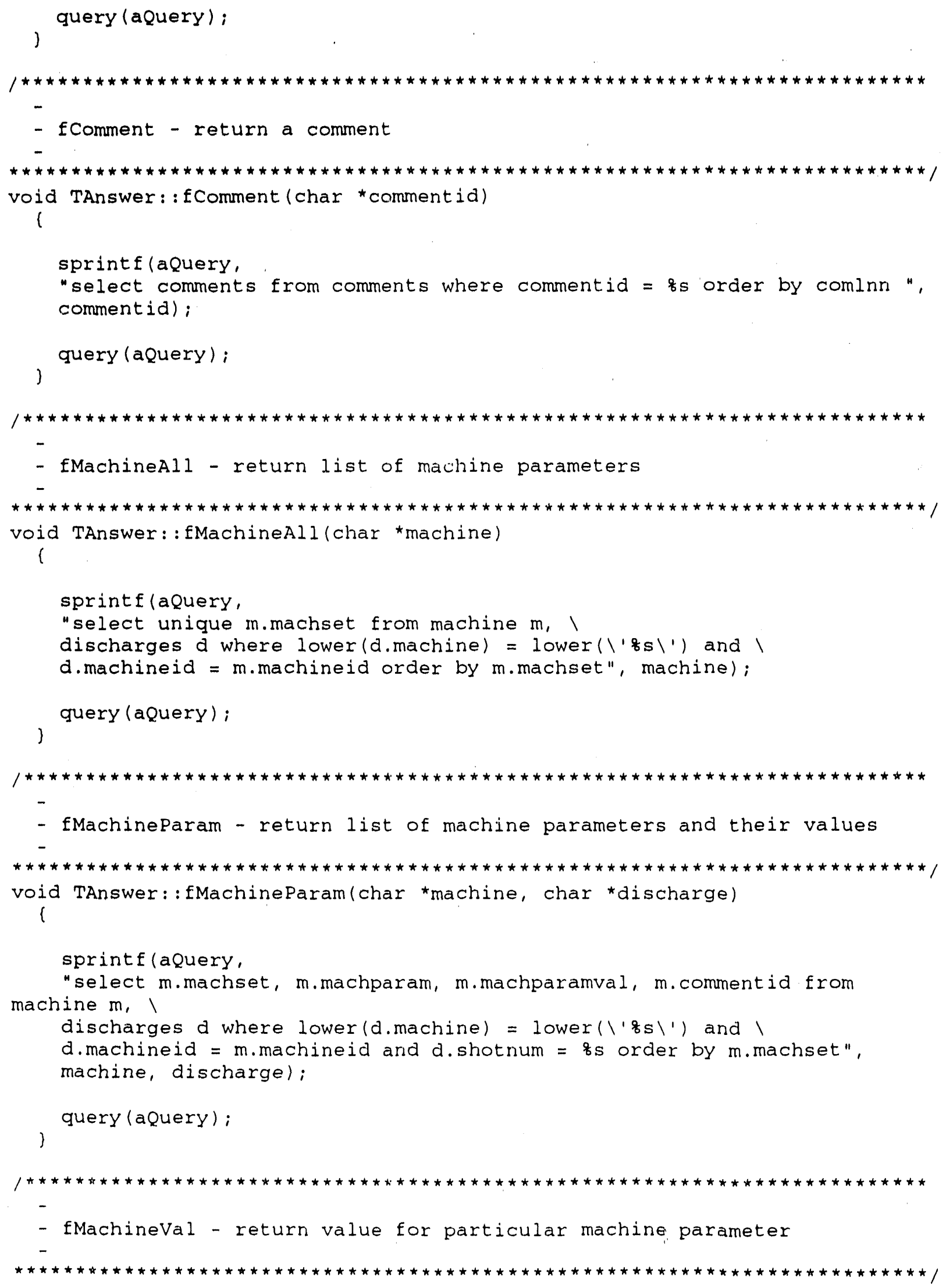




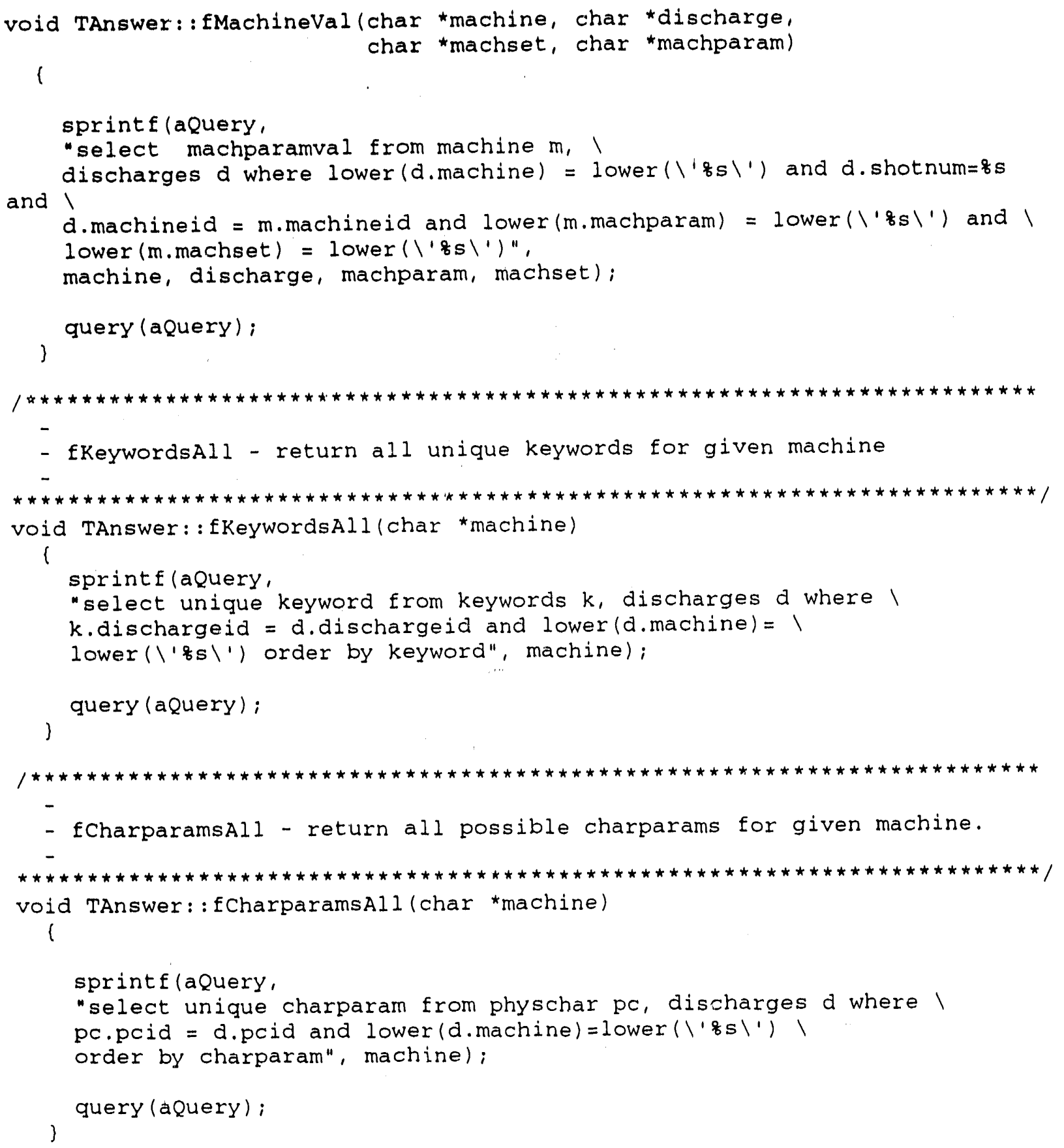




\section{Appendix IV - mdi.m listing}

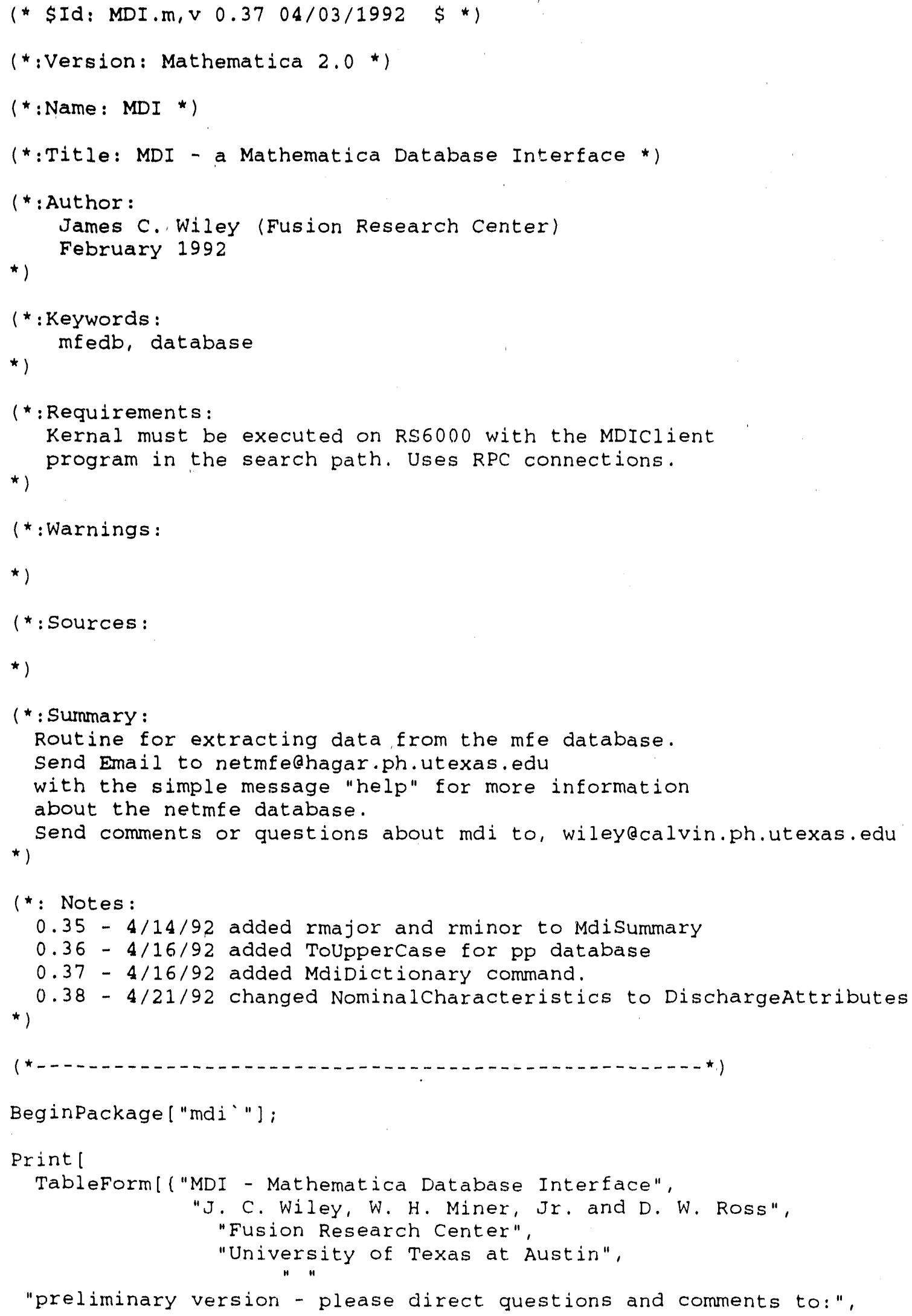


"wileyecalvin.ph.utexas .edu", "or", "minerehagar.ph.utexas.edu"\}, TableAlignments->Center])

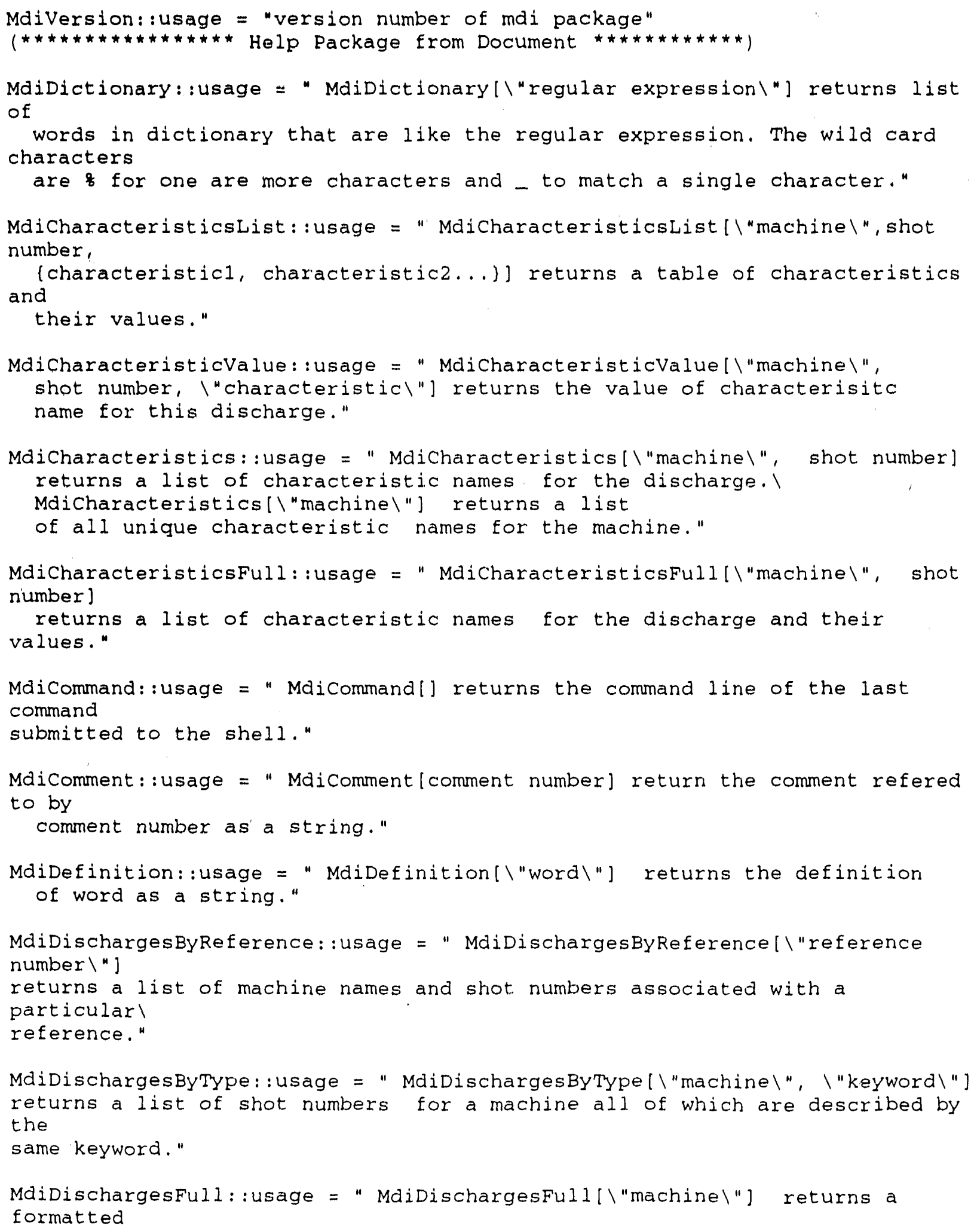


table of shot number $s$ for given machine including the characterization data for

each discharge."

MdiDischargesTable: :usage = "MdiDischargesTable[ "machinel"] returns a table of shot numbers with characterization data including column headings."

MdiDischarges: Iusage $=$ "MdiDischarges[ "machinel" ] returns a 1 ist of shot।

numbers for given machine."

MdiErrorMessage: :usage $=$ "MdiErrorMessage[] - If the last query generated

an

error message from the database engine or from the RPC communication layer, this

command can be used to retrieve the full text of the message."

MdiHeadings: :usage $=$ "MdiHeadings[] returns the DBMS table headings from the

last command."

MdiKeywords: :usage $=$ "Mdikeywords[\"machine\", shot number] returns a list of

key words describing the discharge."

MdiKeywords: :usage $=$ "MdiKeywords[\"machine\"] returns a list of all unique

key words for the machine."

MdiLastQuery: :usage $=$ "MdiLastQuery[] returns the exact form of the last query submitted."

MdiMachineParameters: :usage = "MdiMachineParameters [ "machinel"] returns a list of the names of parameters describing the experimental device that do not change with shot number and their values."

MdiMachines: :usage $=$ "MdiMachines[] requires no argument and returns a list

of machines in the database."

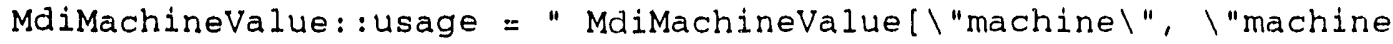
parameter "]

returns the value of a particular machine parameter."

MdiNeIf: :usage = "MdiNeIF[\"machine $"$ ", shot number, slice:1] returns the interpolating function for generic ne profile. "

MdiNePlot::usage = "MdiNePlot [\"machinel", shot number, slice:1] returns the generic ne profile."

MdiDischargeConditionsTable: : usage = " MdiDischargeConditionsTable[ $\backslash$ "machine $"$ ", shot number] returns a table of discharge characteristics with headings for

a particular shot."

MdiDischargeConditions: :usage = "MdiDischargeConditions[ "machine】", 
shot number] returns the nominal Bt, Ip, NeBar, VLoop, Gas, Zeff, and number of time slices for a given discharge in the indicated order."

MdiPauxIF: iusage = "MdiPauxIF[\"machinel", shot number, slice:1] returns the

interpolating function for generic auxiliary power profile."

MdiPauxPlot: :usage = "MdiPauxPlot [ "machine\", shot number, s1ice:1]

returns

the generic auxiliary power profile."

MdiPerturbationstable: :usage = "MdiPerturbationstablel|"machine\", shot number ]

returns a formatted table with headings of the perturbation information."

MdiPerturbations: :usage = "MdiPerturbations[ ["machine\", shot number]

returns a

table of perturbation information."

MdiPhysparamAtTime: :usage = "MdiPhysParamAtTimel I"machinel", shot number, proifle,

position] returns value of profile at given position."

MalPohIF: :usage = "MaiPohIF["machinel", shot number, slice:1] returns the interpolating function for generic ohmic power profile."

MdiPohPlot::usage $=$ "MdipohPlot [\"machine\", shot number, slice:1] returns the

generic ohmic power profile."

MdiprofileData: :usage = "MdiprofileDatal "machinel", shot number, ("profile\")

returns information about the profile."

MdiProfileIf: :usage = "MdiProfileIfl\"machinel", shot number, \"profile\", slice: 1 ],

returns an interpolation furction for a profile."

MdiProfileplot::usage = "Mdiprofileplot (\"machine)", shot number,

\"profile\",

slice:1] returns a graphic object including error bars if available."

MdiProfilesize: :usage = "Mdiprofilesizel "machinel", shot number,

\"profile\",

slice:1] returns the number of points in a profile."

MdiProfiles: :usage = "MdiProfiles[\"machine\", shot number] returns a

list of

profile names for this discharge."

MdiProfileTable: :usage = "MdiProfileTable[ "machine\", (shot number list), (proifle list)] returns a table of profile names for each shot on the list in

which each has the same radial points."

MdiProfilexLabel::usage = "MdiProfilexLabell "machinel", shot number, ("profile\") 
returns the name of independent variable for the profile."

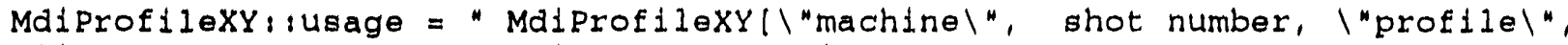
slice:1] returns the a list of $x-y$ pairs."

Mdiprofilefit: :usage = "Mdiprofilefitl"machine】", shot number, $\mid$ "profile|",

slice:1] returns the a list of profile fit parameters."

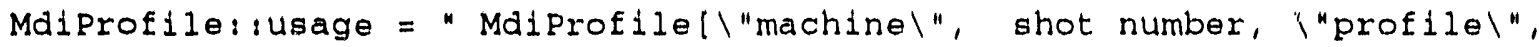
slice:1]

returns a list of $x, d x, y, d y$ four-tuples."

MdiPSPrint: :usage = "MdiPSPrint [ -graphic- ] sends postscript graphics to a particular Apple Laser Writer printer. "

Mdiquery: :usage $=$ "Mdiquery [ "Query... \"] submits the query given as a text

string to the database and returns the resulting table in Mathematica list form."

MdiRawReturn: :usage $=$ "MdiRawReturn[] returns the unprocessed result from the

MDIClient program."

MdiReferences: :usage $=$ "MdiReferences [ "machine\"] returns a list of reference

numbers and the references associated with a particular machine."

MdiRows: : usage $=$ "MdiRows[] returns the number of rows in the last query result

table."

Mdisource: :usage $=$ "MdiSource[ $\backslash$ "machinel", shot number, \"profile\"]

returns

information about the person or group responsible for this measurement."

Mdisummary: :usage = "Mdisummary [\"machine\", shot number] which returns a graphic array of plots for Te, TA, ne, Poh, Paux and a table of the nominal discharge parameters."

MdiToNumber: : usage = "MdiToNumber[\"string\"] - returns number from string." MdiTeIF: :usage = "MdiTeIFl\"machinel", shot number, slice:1] returns the interpolating function for generic Te profile."

MdiTePlot: :usage = "MdiTePlot (\"machine\", shot number, slice:1] returns the generic Te profile."

MdiTiff: :usage = "MdiTiIf[\"machine\", shot number, slice:1] returns the interpolating function for generic Ti profile."

Mditiplot: :usage = "MdiTiplot ["machinel", shot number, slice:1] returns the

generic Ti profile." 


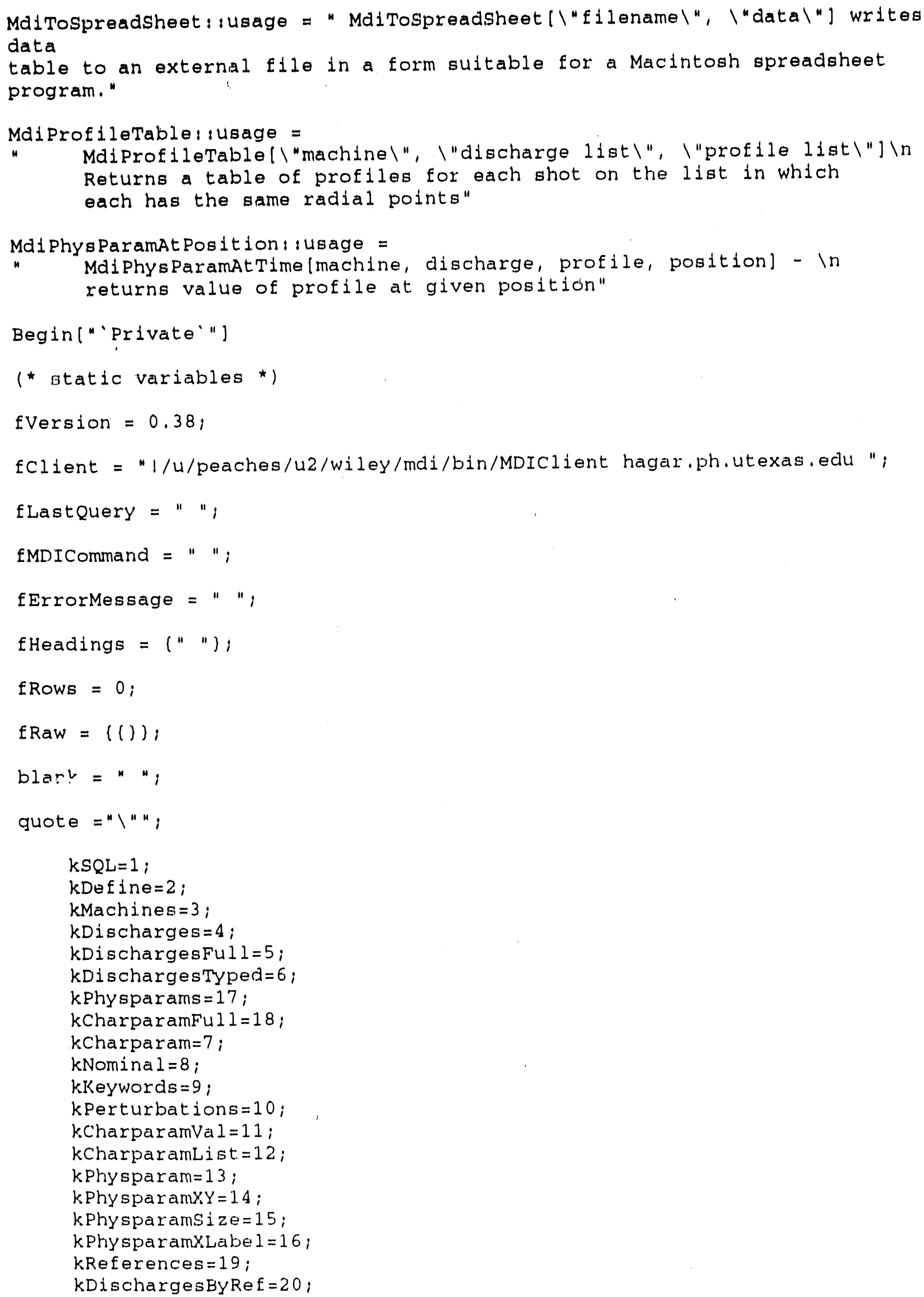




\section{ksource $=21$,}

kPhysparamData $=22$,

kComment $=23$,

kKeywordsAl $1=24$,

kMachineAll $=25$,

kMachineParam $=29$,

kMachineval $=26$,

kCharparamsAl1 $=27$,

kPhysparamFit $=28$,

(* static functions *)

dollarfix[p_string $:=\operatorname{stringReplace}(p,(" \$ "->" \backslash \$ ")]$

stringLiatQ $\left[x_{-}\right):=\operatorname{Apply}(\operatorname{And}, \operatorname{Map}[\operatorname{StringQ}[\#] \varepsilon, x])$

SetAttributes (MdiToNumber, Listable)

(* the get functions make calls to the MDI rpc service functions

they are not exported and should not be used outside of mdi*)

getQuery (aQuery_string] : =

(fMDICommand=stringJoin (fClient, blank, Tostring[kSQL), blank, quote, dollarfix(aquery), quote];

Get (EMDICommand) )

getWord(word_string): =

(fMDICommand=StringJoinl fClient, blank, Tostring[kDefine], blank, quote, dollarfix[word], quote],

Get (fMDICommand])

getMachines[]:=

(EMDICommand=stringJoinl fClient, blank, Tostring(kMachines), blank,

"\" \" "];

Get ( fMDICommand])

getDischarges [machine_string]: =

(fMDICommand=StringJoin [ fClient, blank, Tostring[kDischarges],

blank, quote, machine, quotel;

Get (fMDICommand])

getDischargesFull(machine_string): =

(fMDICommand=stringJoinl fClient, blank, Tostring[kDischargesFul1],

blank, quote, machine, quotel;

Get (fMDICommand])

getDischargesTyped [machine_string, type_string] :=

( $\mathrm{fMDIC}$ Comand=stringJoinl fClient, blank, Tostring(kDischargestyped), blank, quote, machine, blank, type, quotel;

Get (fMDICommand))

getcharacteristics [machine_string]: =

(fMDICommand=stringJoinl fClient, blank, ToString(kCharparamsAld), blank, quote, machine, quotel;

Get (fMDICommand]) 
getcharacteristics[machine_string, discharge_String] :=

(fMDICommandastringjoinl fClient, blank, Tostring(kCharparam), blank, quote, machine, blank, discharge, quotel,

Get ( EMDICommand])

getcharacteristiceFuli (machine_string, diacharge_string] : =

(fMDICommand=StringJoinl fClient, blank, Tostring(kCharparamFul1),

blank, quote, machine, blank, discharge, quotell

Get (EMDICommand])

getcharacteristicVal (machine_string, discharge_string, charparam_string):= (EMDICommand=stringJoinl fClient, blank, ToString[kCharparamval], blank, quote, machine, blank, discharge, blank, dollarfix (charparam], quote],

Get (EMDICommand])

getcharacteristicsList (machine_string, discharge_string, pestringList_String]: =

(fMDICommand=StringJoin ( ECIlent, blank, Tostring(kCharparamList), blank, quote, machine, blank, lischarge, blank, dollarfix(postringlist), quote);

Get (fMDICommand])

getDischargeConditions (machine_string, discharge_string]: = (fMDICommand=StringJoinl fClient, blank, Tostring(kNominal), blank, quote, machine, blank, discharge, quote]; Get ( fMDICommand])

getKeywords [machine_string, discharge_string]: = (fMDICommand =stringJoinl fClient, blank, Tostring[kKeywords], blank, quote, machine, blank, discharge, quotel; Get ( fMDICommand])

getPerturtations[machine_string, discharge_string]:= (fMDICommand=StringJoinl fClient, blank, Tostring[kPerturbations], blank, quote, machine, blank, discharge, quote], Get (fMDICommand])

getprofilelmachine_string, discharge_string, profile_string, slice_Integer:1]:= (fMDICommand=StringJoin [ fClient, blank, TostringlkPhysparam], blank, quote, machine, blank, discharge, blank, dollarfixlprofile], blank, Tostring[slice], quote];

Get (fMDICommand])

getProfilexy[machine_string, discharge_string, profile_string, slice_Integer:1]:= (fMDICommand=StringJoin ( $\mathrm{fClient,blank,} \mathrm{Tostring[kPhysparamXY],}$ blank, quote, machine, blank, discharge, blank, dollarfix[profile], blank, Tostringlslice], quotel;

Get ( fMDICommand] )

getProfilefit/machine_string, discharge_string, profile_string, slice_Integer:1]:= (fMDICommand=stringJoin ( fClient, blank, Tostring[kPhysparamFit], blank, quote, machine, blank, discharge, blank, dollarfix[profile], blank, Tostring[slice], quote], Get (fMDICommand]) 
getprofilesizelmachine_string, discharge_string, profile_string, slice_Integer:11: =

(EMDICommand=StringJoin [ fClient, blank, Tostring[kPhysparamsize], blank, quote, machine, blank, discharge, blank, dollarfix(profile], blank, Tostringlslice], quocel),

Get ( IMDICommand])

getprofilexLabel (machine_string, discharge_string, profile_string, slice_Integer:11:=

(fMDICommand=stringJoin [ fClient, blank, Tostring (kPhysparamXLabel], blank, quote, machine, blank, discharge, blank, dollarfix(profile), blank, Tostring[slice], quotel)

Get ( fMDICommand])

getPhysParams [machine_string, discharge_string] := (fMDICommand=StringJoin [ fClient, blank, Tostring(kPhysparams], blank, quote, machine, blank, discharge, quotej; Get (fMDICommand])

getReferences [machine_string]: =

(fMDICommand=StringJoin [ fClient, blank, Tostring[kReferences],

blank, quote, machine, quotel:

Get (fMDICommand])

getDischargesByReference[refid_string]:=

(fMDICommand=stringJoinl fClient, blank, TostringlkDischargesByRef), blank, quote, refid, quotel;

Get (fMDICommand])

getSource[machine_string, discharge_string, profile_string]:= (EMDICommand=StringJoin [ fClient, blank, ToString[kSource], blank, quote, machine, blank, discharge, blank, dollarfix[profile], quote], Get (fMDICommand] )

getProfileDatalmachine_string, discharge_string, profile_string, slice_Integer:11: =

(fMDICommand=StringJoin! fClient, blank, ToString[kPhysparamData] blank, quote, machine, blank, discharge, blank, dollarfix[profile], blank, Tostring(slice], quote];

Get [ fMDICommand] )

getComment [commentid_string]:=

(fMDICommand=StringJoin [ fClient, blank, Tostring[kComment], blank, quote, commentid, quotel;

Get (fMDICommand])

getMachineAll[machine_string]: =

(fMDICommand=StringJoinl fClient, blank, Tostring[kMachineAl1],

blank, quote, machine, quote];

Get (fMDICommand])

getMachiraParameters [machine_string, discharge_string] := (fMDICommand=stringJoin [ fClient, blank, Tostring[kMachineParam], blank, quote, machine, blank, discharge, quote]; Get ( fMDICommand])

getMachineValue[machine_string, discharge_string, machineset_string, 
machineParameter_St.ring] : = (fMDICommand=stringJoin [ fClient, blank, Tostring[kMachineVal], blank, quote, machine, blank, discharge, blank,

dollarFix[machineset], blank, dollarfix[machineparameter], quote]; Get [ fMDICommand] )

getKeywords [machine_string]: = (fMDICommanr $=$ StringJoin [ fClient, blank, Tostring[kKeywordsAll], blank, quote, machine, quotel; Get [ fMDICommand] )

$(*$ Mdiclean takes the raw list returned by MDIClient and tries to determine if a query result or error message was returned. If a query is returned then the first row should be a string with the query echoed having the first

four characters ANSQ. If there is a query returned, then the second to last row often contains the message $\mathrm{xx}$ rows selected or no rows selected. If the query was executed but no rows were returned, then this is indicated;

otherwise, the result table is processed and headings are stripped and stored. If an error occured in the RPC communication, then an RPC *) error message is returned and the full error message is stored.

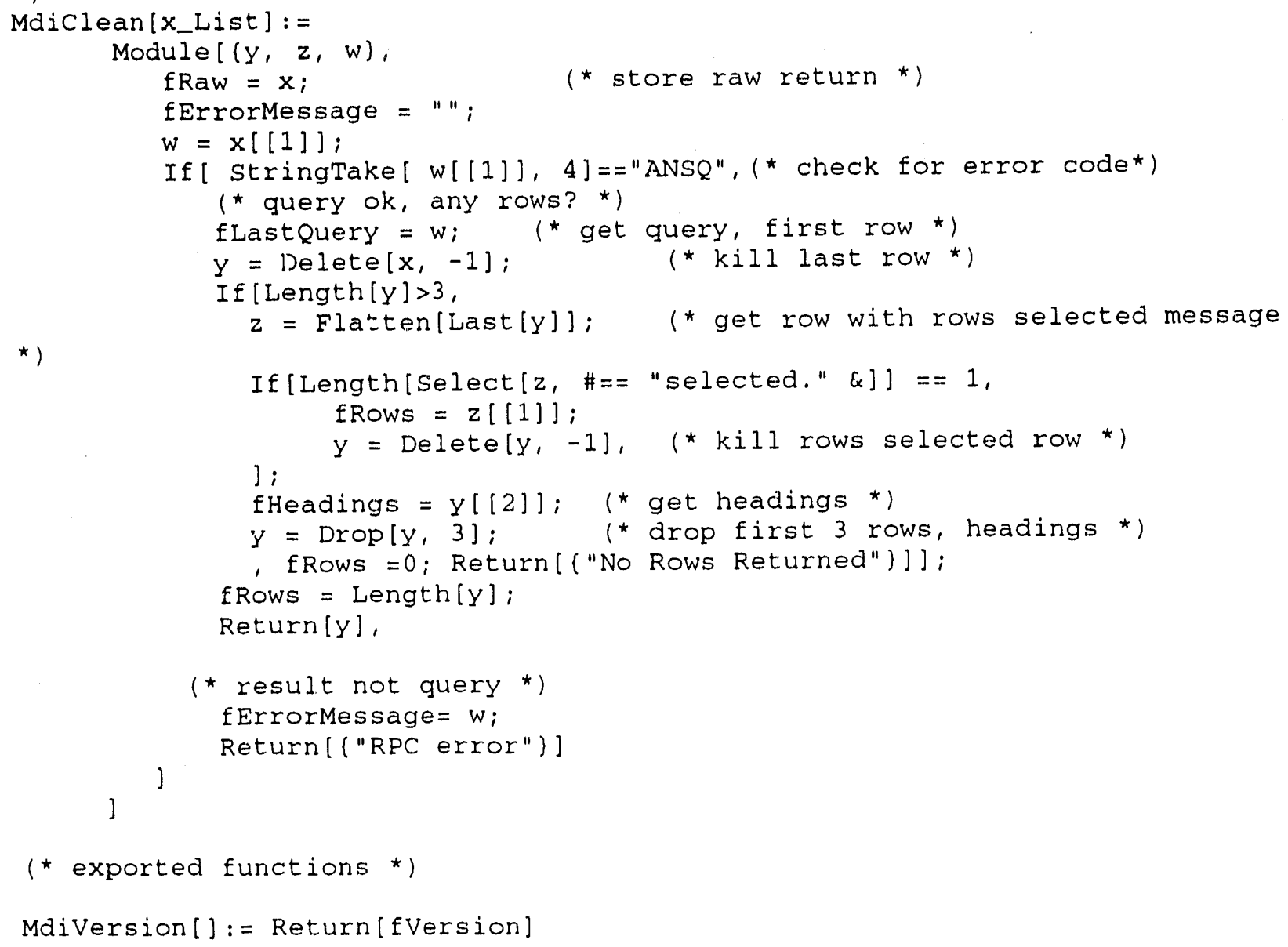




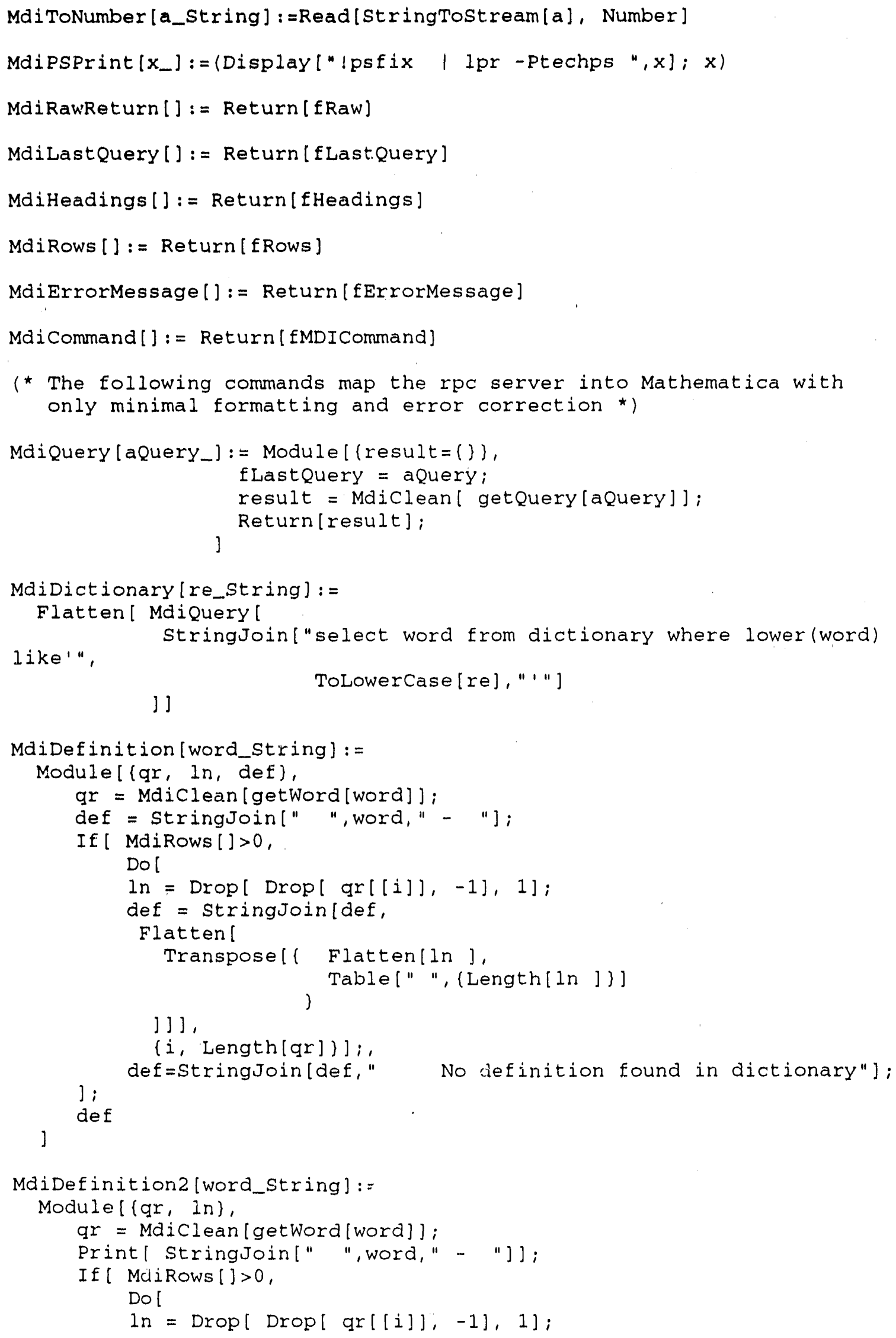




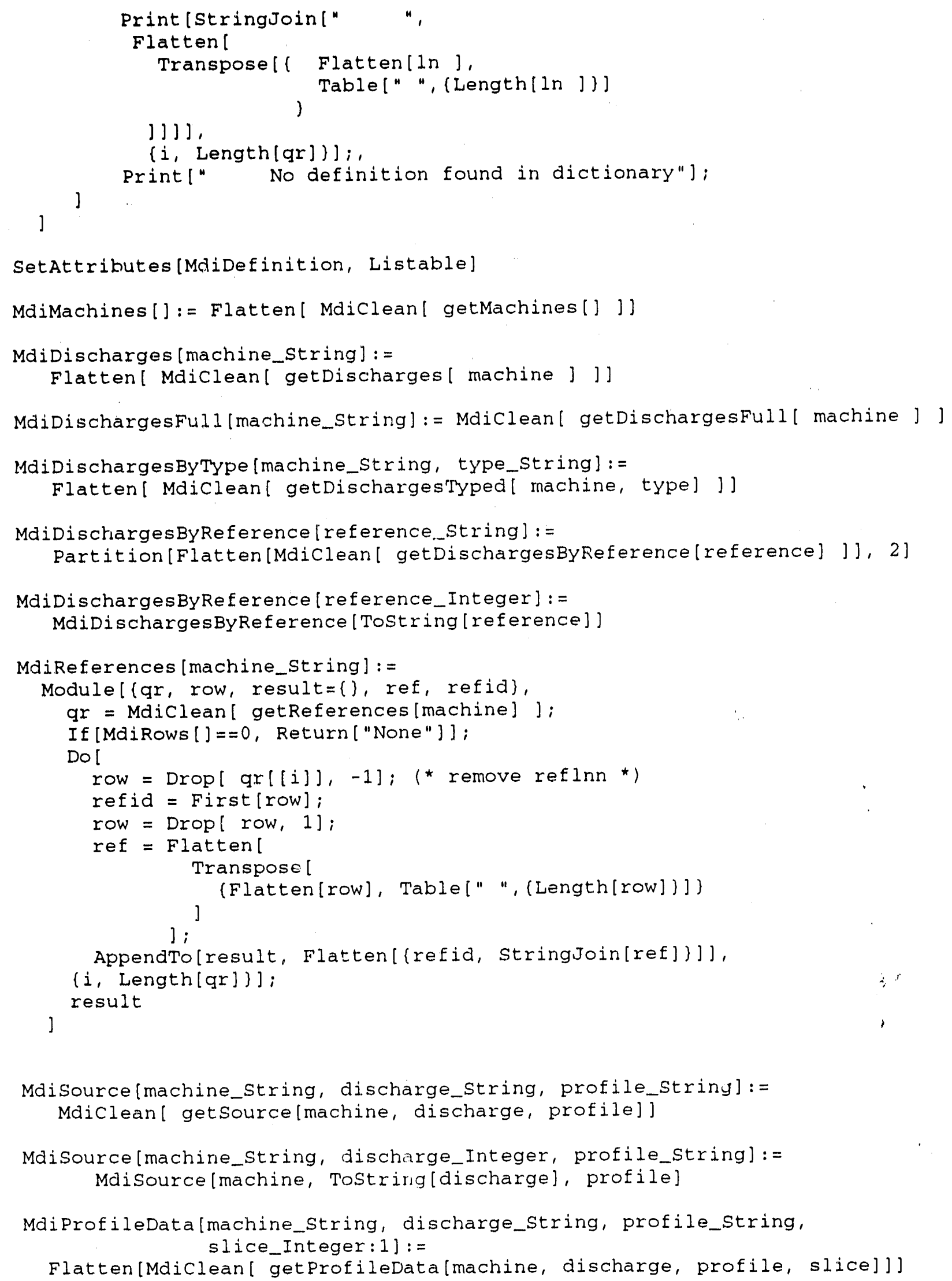


MdiProfileDatalmachine_string, discharge_Integer, profile_String, slice_Integer:1]:= Mdiprofiledata[machine, Tostring[discharge], profile, slice]

Mdicomment [n_Integer] : =

Module [(gr, ln, def $\}$, $\mathrm{gr}=\operatorname{Mdiclean}[$ getComment $[$ Tostring[n]]];

def = StringJoin["Comment "Tostring[n]," - "];

If [ MdiRows []>0,

Dol

ln $=$ gri

def = StringJoin!def,

Flatten !

Transpose[\{ Flatten[1n], )

Table [" ", (Length[1n])]

] ] ,

$\{i$, Length $[\mathrm{gr}]\}] ;$, def=StringJoin [def,"

No definition found in dictionary"];

]'i

]

def

SetAttributes [Mdicomment, Listable]

MdiMachineParameters [machine_String]:=

Mdiclean[ getMachineAll[machine] ]

MdiMachineParameters [machine_string, discharge_string] : = Mdiclean[ getMachineParameters[machine, discharge] ]

MdiMachineParameters [machine_Sering, discharge_Integer] := MdiMachineParameters[machine, Tostring[discharge]]

MdiMachineValue[machine_string, discharge_string, machineset_string, machineParam_string]:=

Flatten[ Mdiclean! getMachinevaluelmachine, discharge, machineset, machineParam] ]]

MdiMachineValue [machine_String, discharge_Integer, machineset_String, machineParam_String]:= MdiMachinevalue [machine, Tostring[discharge], machineset, machineparam]

Mdicharacteristics [machine_string] : =

Flatten[Mdiclean[ getCharacteristics [machine] ]]

MdiCharacteristics [machine_string, discharge_String]:= Flatten[ Mdiclean[ getCharacteristics[ machine, discharge] ]]

Mdicharacteristics [machine_String, discharge_Integer] : = Mdicharacteristics[ machine, Tostring[discharge]]

MdicharacteristicValue [machine_string, discharge_string, charparam_string]:=

Flatten[ Mdicleanl getCharacteristicval[machine, discharge, charparam] ]] 
Mdicharacteristicvalue [machine_string, discharge_Integer,
charparam_string] := MdicharacteristicValue [machine, Tostringldischarge], charparam]

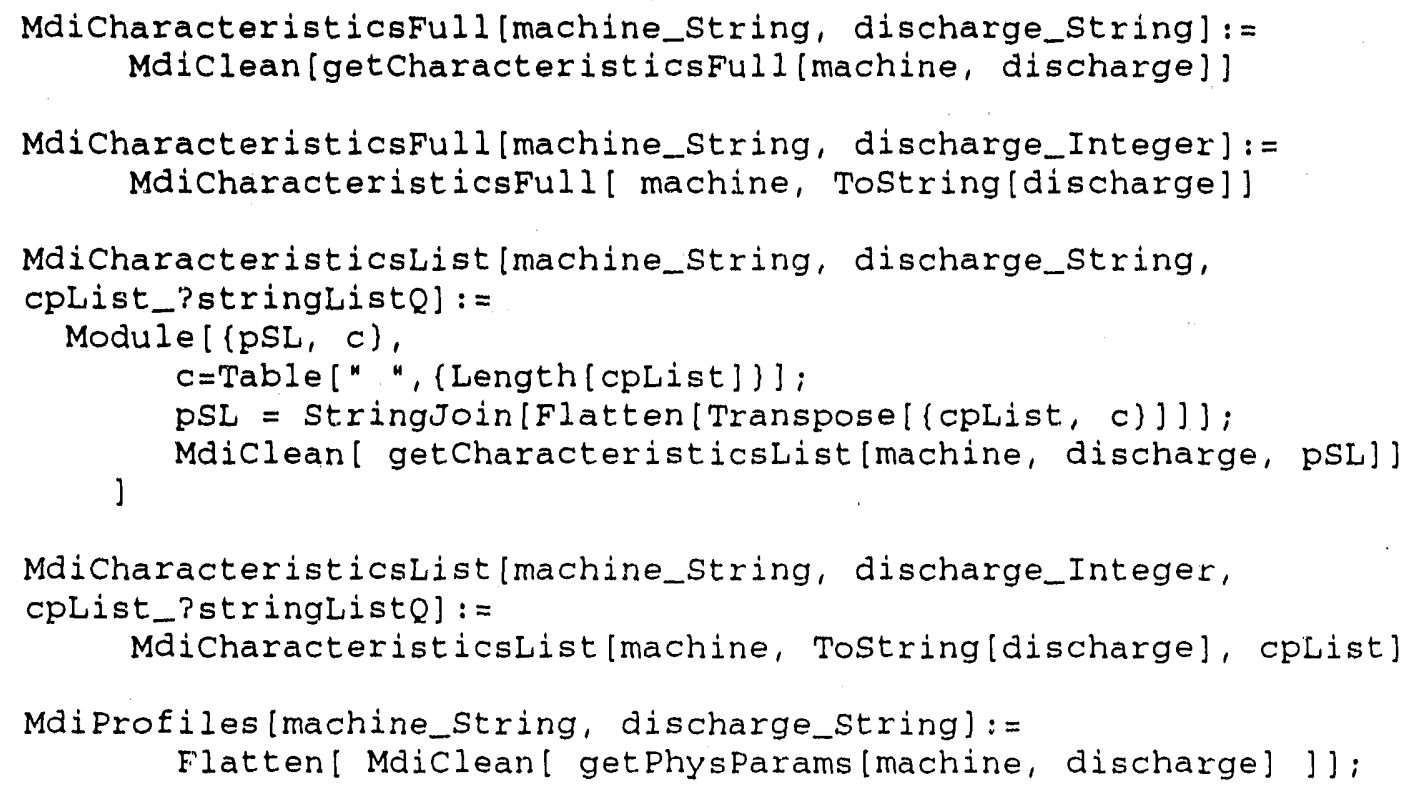

MdiDischargeconditionsTable[machine_string, discharge_Integer] := MdiDischargeConditionsTable [machine, Tostring[discharge]]

Maikeywords [machine_string, discharge_string] : = Flatten[ Mdiclean[ getKeywords[machine, discharge] ]]

Mdikeywords [machine_String, discharge_Integer] : = Mdikeywords [machine, Tostring[discharge] ]

Mdikeywords [machine_string] : = Flatten[ Mdiclean[ getKeywords[machine] ]]

MdiPerturbations [machine_String, discharge_string]:= Mdiclean[ getperturbations [machine, discharge] ] 


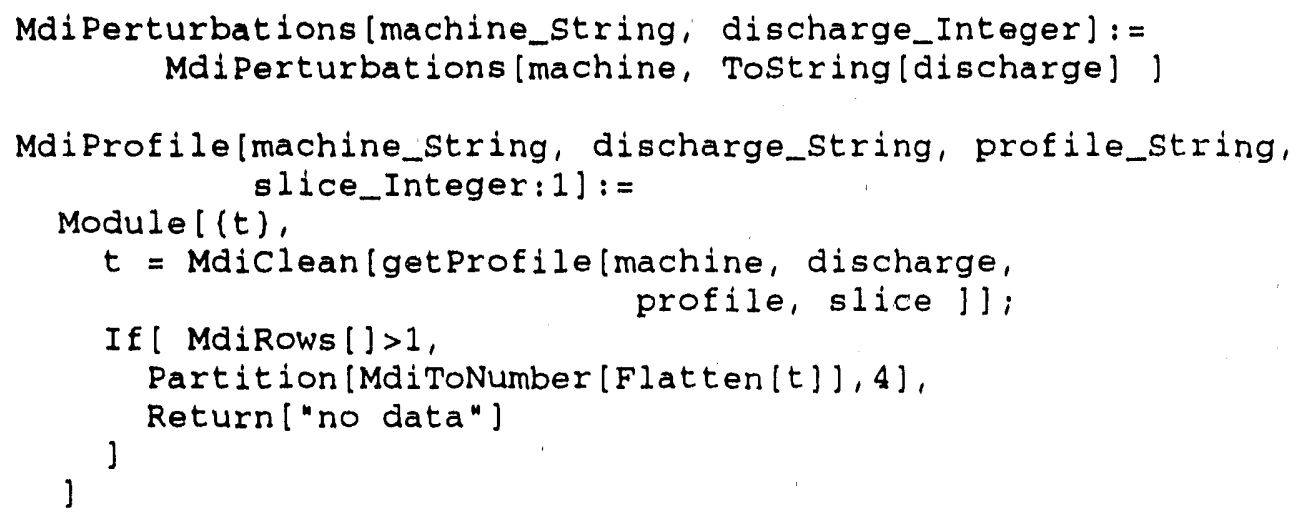

Mdiprofilelmachine_string, discharge_Integer, profile_string, slice_Integer:1]:=

Mdiprofilél machine, Tostring[discharge], profile, slice]

Mdiprofilexy[machine_string, discharge_string, profile_string, Module [ $(t)$, slice_Integer:1]:=

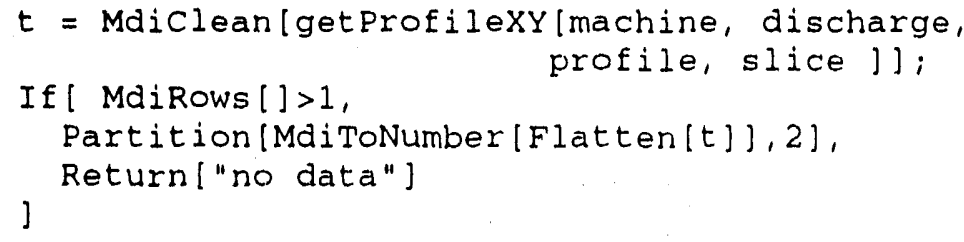

MdiProfilexy[machine_string, discharge_Integer, profile_string, slice_Integer: 1$]:=$

MdiprofilexY[ machine, Tostring[discharge], profile, slice]

Mdiprofilesizelmachine_string, discharge_string, profile_string, slice_Integer: 1$]:=$

Flatten[ Mdicleanl getProfilesizelmachine, discharge, profile, slice] ]);

MdiProfilesizelmachine_string, discharge_Integer, profile_string, slice_Integer: 1$]:=$

Mdiprofilesize[machine, Tostringldischarge], profile, slice]

MdiProfilefitlmachine_string, discharge_string, profile_string, slice_Integer: 1$]:=$

Flatten[ Mdicleanl getprofileFit (machine, discharge, profile, slice] J];

MdiProfilefitlmachine_string, discharge_Integer, profile_string, slice_Integer:1]:=

Mdiprofilefit (machine, Tostring[discharge], profile, slice]

MdiProfilexLabel lmachine_string, discharge_string, profile_string, slice_Integer $: 1]:=$

Flattenl Mdicleanl getProfilexLabellmachine, discharge, profile, slice] J]; 
MdiprofilexLabel[machine_string, discharge_Integer, profile_string, slice_Integer:1]:= MdiprofilexLabel[ machine, Tostringldischarge], profile, slice]

(* The following commands use the basic Mdi commands to construct new functions *)

MdiDischargesTable [machine_string]: =

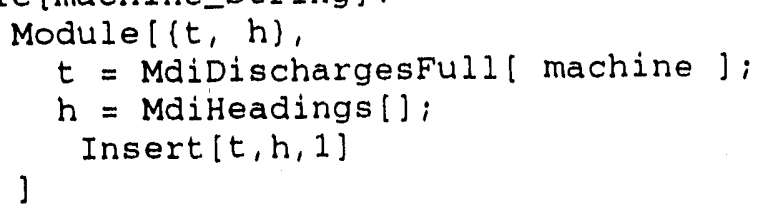

MdiperturbationsTable[machine_string, discharge_string]: = Module $[(t, h]$, $t=$ MdiPerturbations [ machine, discharge ]; $\mathrm{h}=$ MdiHeadings [];

MdiPerturbationsTable [machine_string, discharge_Integer]:= MdiPerturbationsTable [machine, Tostring[discharge] ]

MdiprofileIflmachine_string, discharge_string, profile_string,

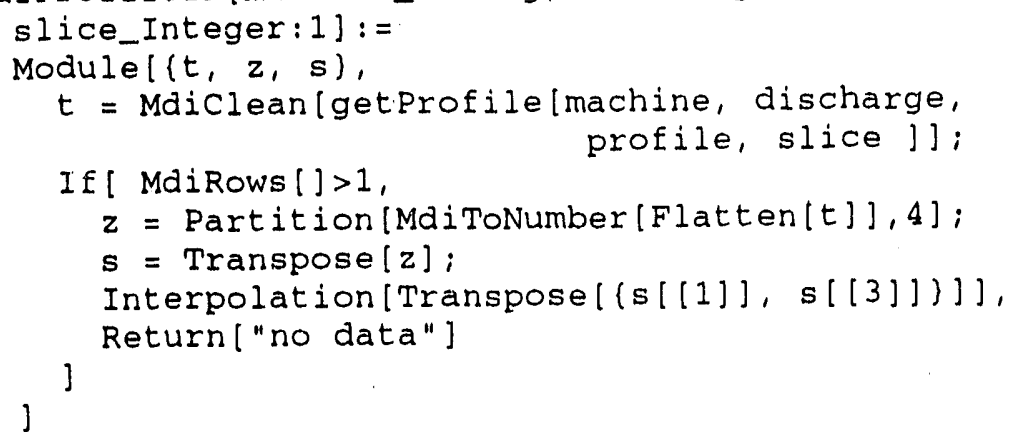

MdiprofileIf[machine_String, discharge_Integer, profile_string, slice_Integer: 1$]:=$

MdiprofileIF[ machine, Tostring[discharge], profile, slice]

$g\left[\left(x_{-}, d x_{-}, y_{-}, d y_{-}\right\}\right):=\{(\{x, y+d y\},\{x, y-d y\}),\{(x+d x, y\},\{x-d x, y\})\}$

boundary[] : = Line $[\operatorname{Map}[$ Scaled, $(\{0.01,0.01\},(0.99,0.01\},(0.99,0.99)$, $\{0.01,0.99\},(0.01,0.01\})\}]$

Mdiprofileplot(machine_string, discharge_string, profile_string, slice_Integer: 1$]:=$

Module $[\{t, z, s, x$ Label, scal, fac, yLabel $\}$, $z=$ Mdiprofile [machine, discharge, profile, slice];

If [ MdiRows[] $>1$,

$\left({ }^{*} z=\right.$ Partition [MdiToNumber $[$ Flatten $\left.\left.[t]], 4\right] ; *\right)$

$s=\operatorname{Transpose}[z] ;$

sca $]=F l o o r[N[\log [10, \operatorname{Max}[A b s[s[[3]]]]]]] ;$

If $[$ Abs [scal] $>2$, 


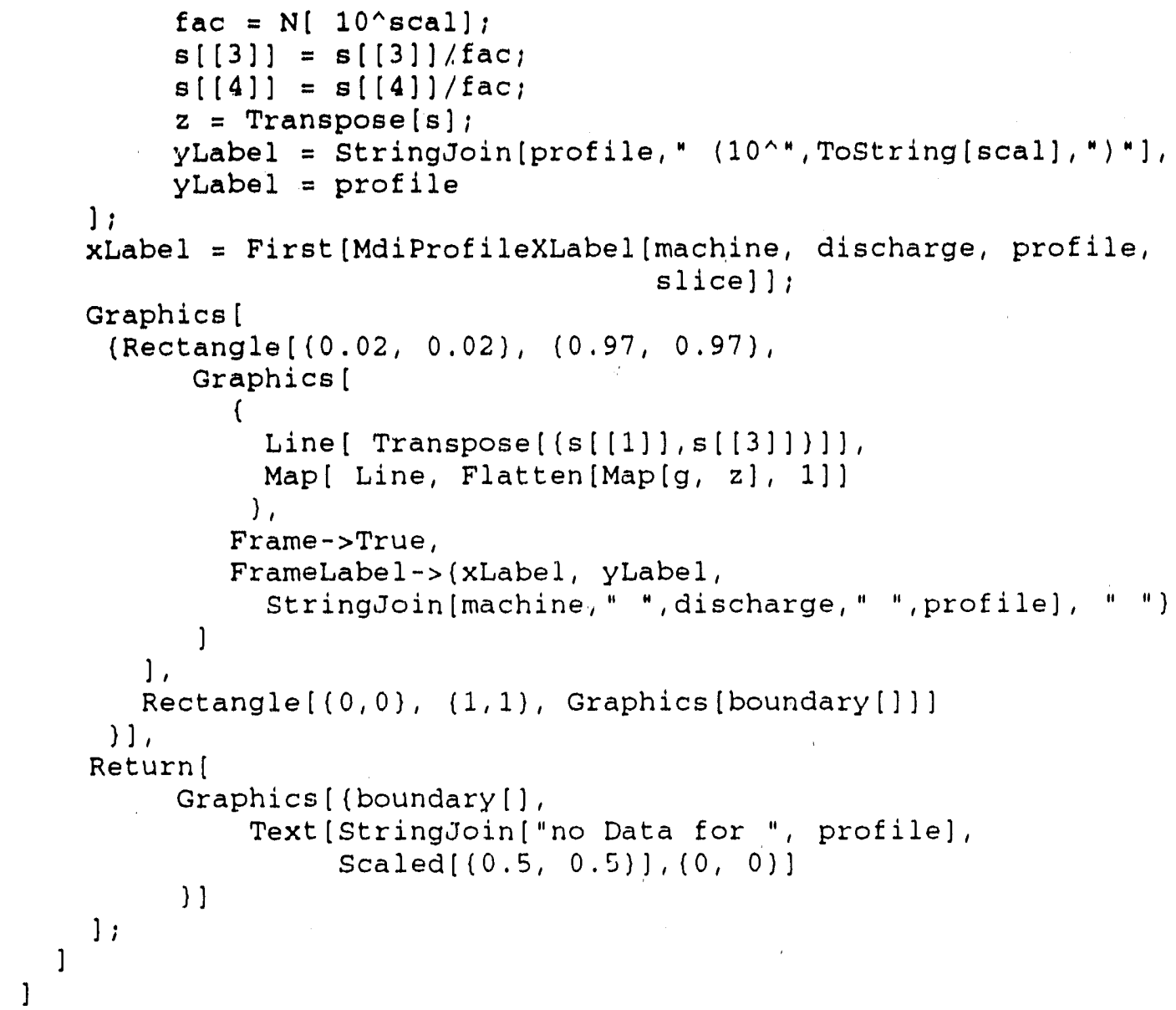




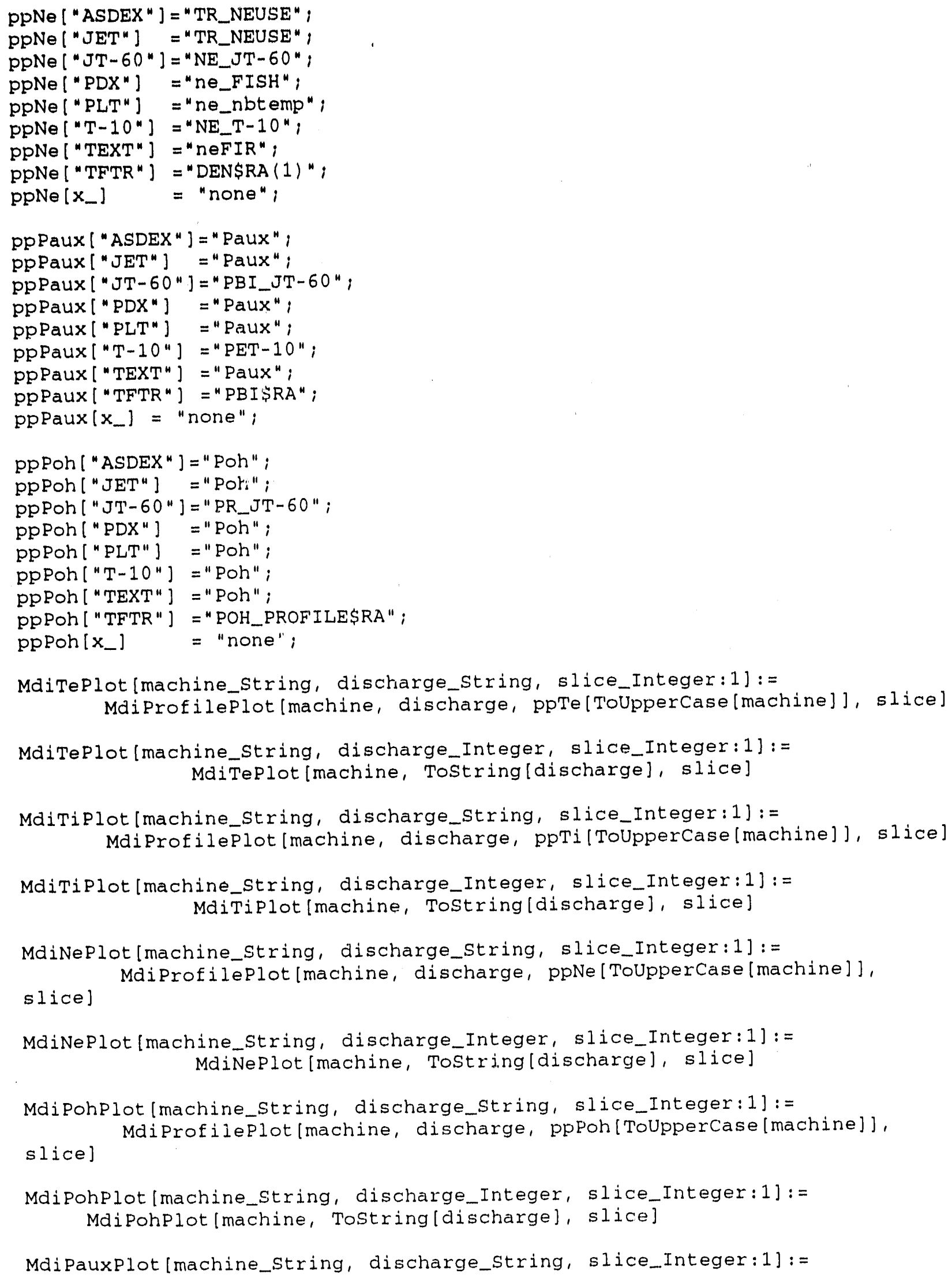




\section{slice]}

Maiprofileplot [machine, discharge, ppPaux[Touppercase[machine]),

MdiPaux.Plot [machine_string, discharge_Integer, slice_Integer:1]:=

Mdipauxplot (machine, Tostring(discharge], silce)

MdiTeIF[machine_string, discharge_string, slice_Integer:1]:=

MdiprofileIF[machine, discharge, ppTe[ToUpperCase[machine]], slice]

MdiTeIF[machine_string, discharge_Integer, slice_Integer:1]:=

MdiTeIf[machine, Tostring[discharge], slice]

MdiTiIF[machine_String, discharge_string, slice_Integer:1]:= MdiProfileIF(machine, discharge, ppTi[ToupperCase[machine]), slice]

MdiTiflmachine_string, discharge_Integer, slice_Integer:1]:= MdiTiff[machine, Tostring[discharge], slice]

MdiNeIF[machine_string, discharge_String, slice_Integer:1]:= MdiprofileIF[machine, discharge, ppNe[ToUpperCase[machine]], slice]

MdiNeIF[machine_string, discharge_Integer, slice_Integer:1]:= MádiNeIF[machine, Tostring[discharge], slice]

MdiPohIF(machine_String, discharge_string, slice_Integer:1]: zt MdiProfileIF[machine, discharge, pppoh(ToupperCase[machine]), slice]

MdiPohIF[machine_String, discharge_Integer, slice_Integer:1]:= MdiPohIF[machine, Tostring[discharge], slice]

MdiPauxIF[machine_string, discharge_string, slice_Integer:1]:= MdiProfileIF[machine, discharge, pppaux[Touppercase[machine]], slice]

MdiPauxIF[machine_string, discharge_Integer, slice_Integer:1]:= MdiPauxIF[machine, Tostring[discharge], slice]

Mdisummary [machine_string, discharge_string] : = GraphicsArray!

(

[Graphics [ (boundary [], Text [StringJoin [machine," ", discharge], scaled $[(0.5,0.97)],(0.1)]$,

Text [ AppendlAppendlAppend! Drop [MdiDischargeConditionstable [machine, discharge], -2$]$,

("rmajor", MdicharacteristicValue[machine, discharge, "rmajor"])],

("rminor", MdicharacteristicValuelmachine, discharge, "rminor"])],

Mdikeywords [machine, discharge] ]//TableForm, scaled $[\{0.05,0.85\}],(-1,1\}]$

)], MdiTeplot [machine, discharge]\},

(MdiNePlot[machine, discharge], MdiTiplot[machine, discharge]),

(Mdipohplot [machine, discharge], Mdipauxplot [machine, discharge])

]

Mdisummary[machine_String, discharge_Integer] : = 


\section{Malsumary (machine, Tostring(discharge)]}
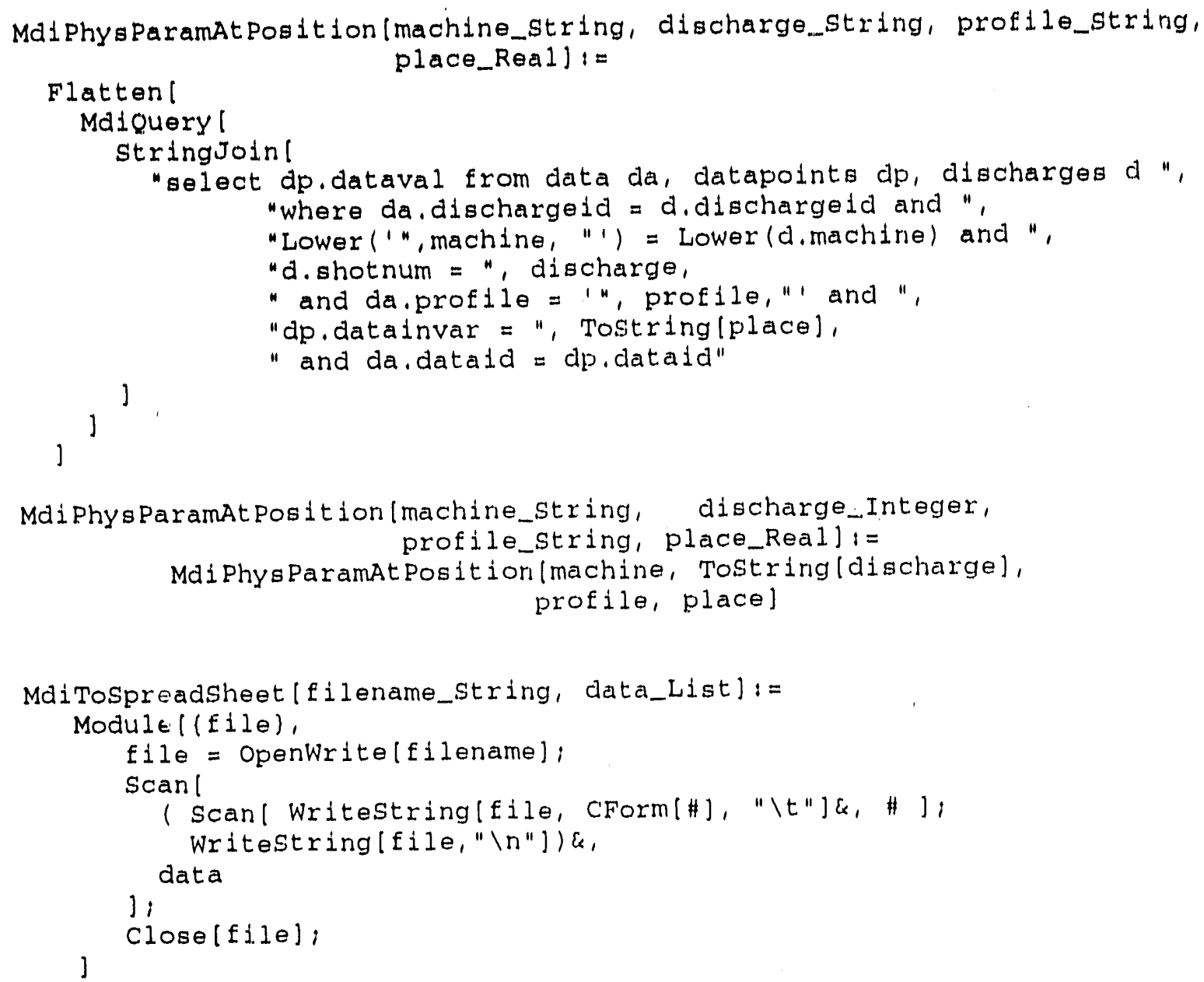

Mdicharacteristicstable[machine_string, shotList_List, pcList_List]:=

Module[(npc, nshots, rt, $q r, \bar{k} \theta y$, value, shot, $i, f]$, $\mathrm{npc}=$ Length [pcList]; nshots = Length [shotList]; 


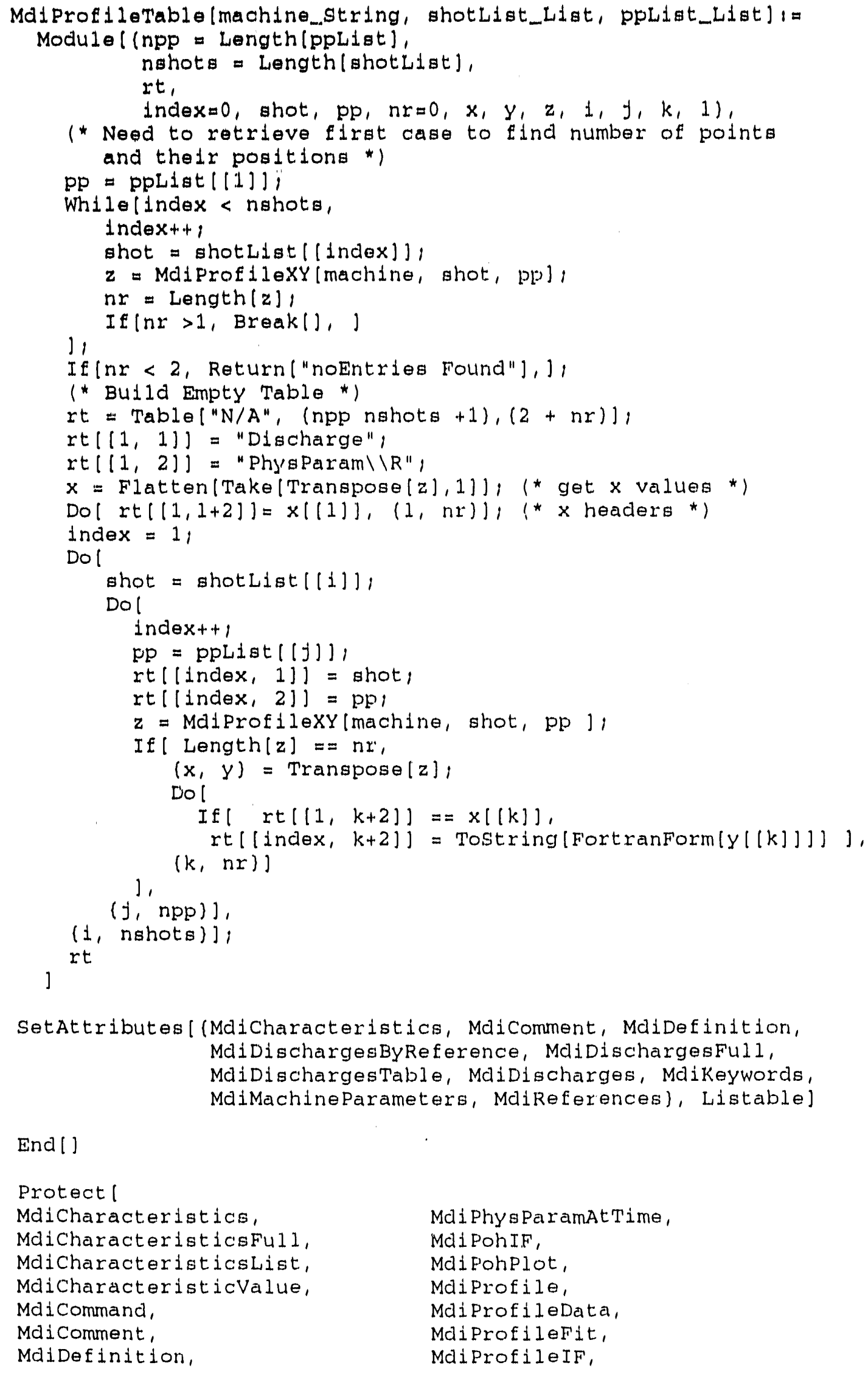

End []

Protect I

Mdicharacteristics, Mdicharacteristicsfull, MdicharacteristicsList, Mdicharacteristicvalue, Mdi Command, Mdi Comment, MdiDefinition,

MdiPhys ParamatTime, MaiPohIF, Mdipohplot, Mdiprofile, MaiProfileData, MdiprofileFit, MdiProfileIF, 
Mdidictionary,

MadDischarges,

MdiDischargeaByReference,

MdiDischargesByType,

MdiDischargeafuli,

Mdidischargestable,

MdiErrorMesaage,

MdiHeudings,

Mdikeyworda,

Mdiluatquery,

MdiMachineParameters,

MdiMachines,

MdiMachineValue,

$M d \perp N e I F$,

MdiNePlot,

MdiDischargeConditions,

Md1DischargeConditionstable,

MdiPauxIF,

Mdi PauxPlot,

Mdiperturbat tons,

Mdi PerturbationsTable,

EndPackage []
MdiprofilePlot, Malprofiles, Mdiprofilesize, MdiprofileTablo, MdiProf 1 lexLabel, MdiproftlexY, Malpsprint, Mdiquery, M11Rawketurn, Mdireferences, MdiRows, Mdisource, Mdisummary, MddTeIF, MdiTePlot, MdiTiIF, MdiTiplot, MdiToNumber, MdiToSpreadsheet, Mdiversion 

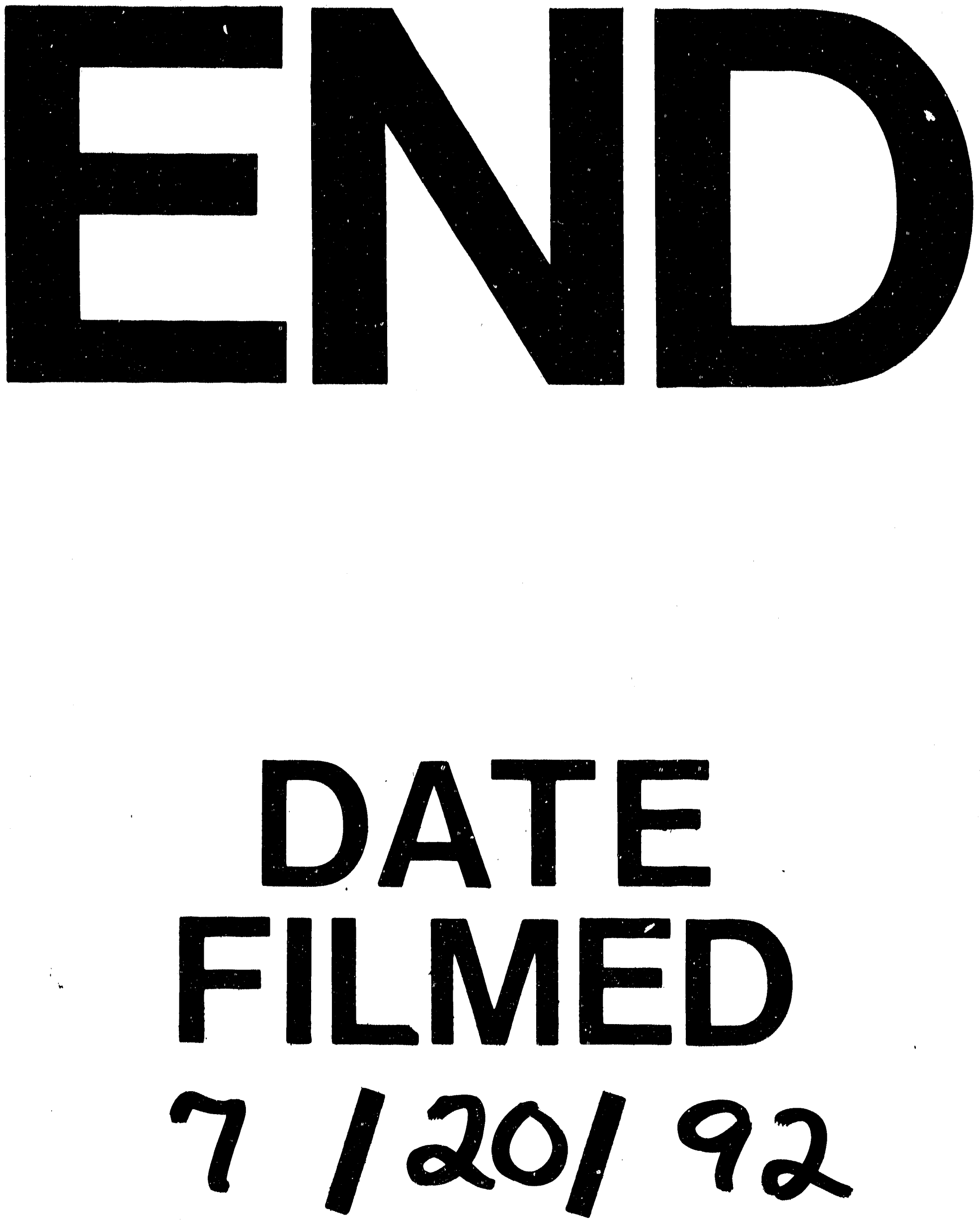